\title{
RESPECTFUL DEFORMATION OF BI-LIPSCHITZ AND QUASISYMMETRIC EMBEDDINGS
}

\author{
Jouni Luukkainen
}

\section{Introduction}

Applying the torus technique of Kirby [Ki], Edwards and Kirby presented in [EK] an alternative to Chernavski1's [Ch] method for deforming embeddings of topological manifolds. They proved Chernavskiri's theorem that if $U$ is an open neighbourhood of a compact set $B$ in a topological manifold $M$, then in the space $E(U ; M)$ of open embeddings of $U$ into $M$ (with the compact-open topology) there is a neighbourhood of the inclusion map id: $U \rightarrow M$ which can be continuously deformed, within a given neighbourhood of id, into the set of embeddings coinciding with id on $B$, in such a way that id is kept fixed and that no changes to embeddings are needed outside a given compact neighbourhood of $B$ in $U$. Furthermore, this theorem was generalized in [EK] to a form which is respectful to a given closed locally flat submanifold $N$ of $M$, in the sense that having $U \cap N$ as the inverse image of $N$ or being, in addition, the identity on $U \cap N$ are properties for an embedding which are preserved under the deformation. As mentioned in [Ch], the generalization also follows from the proof of [Ch]. In [EK] there is also a third proof for these results, which uses a torus technique of Edwards (cf. [Ed]).

Siebenmann [Si] developed Edwards's method further for deforming, in particular, embeddings of locally finite simplicial complexes respectfully to all subcomplexes.

Replacing the torus in Kirby's method by a compact almost parallelizable hyperbolic manifold, Sullivan $\left[\mathrm{Su}_{1}\right]$ proved a nonrespectful analogue of the Chernavski1-Edwards-Kirby theorem for LIP (= locally bi-Lipschitz) embeddings of LIP manifolds without boundary and for LQC (= locally quasiconformal) embeddings of LQC manifolds without boundary. (See $\left[\mathrm{TV}_{2}\right]$ for an exposition of a part of Sullivan's theory.) After this Siebenmann and Sullivan [SS, Appendix B] proved an analogue of the respectful Siebenmann theorem for LIP embeddings of locally finite simplicial complexes. This result implies a respectful version of Sullivan's theorem in the case of LIP embeddings. Furthermore, in [SS] the constructed deformation was shown to preserve LIP isotopies. 
The proof in [SS] is only an outline. The theorem itself is also stated only in an absolute form (as above), not in a full relative one as needed for some applications I have had in mind. The purpose of this paper is to give a detailed presentation of the Siebenmann-Sullivan deformation theory in the (locally) Euclidean case. In $\mathbf{R}^{n}$ and in $\mathbf{R}_{+}^{n}$ our deformations are respectful to all products of coordinate axes and half-axes. The families $\mathcal{Y}_{n}$ and $\mathcal{Y}_{n}^{+}$, respectively, of these products are convenient both for proofs and for applications to manifolds.

Our case allows some simplifications in the proofs (in particular, technical results in the appendices of [SS] are not used) and, more important, it is suitable for LQC embeddings, too. Thus, what we prove, in fact, is a respectful and LIP parametrized version of Sullivan's theory.

It should be noted that Sullivan's proof does not directly generalize to the respectful case as the proof in [EK] does because the hyperbolic manifolds used are not related in a simple inductive manner as are the tori $\left(S^{1}\right)^{n}$.

The reader is not assumed to be familiar with the above-mentioned papers. However, for some constructions based on hyperbolic geometry (and thus on Sullivan's work $\left.\left[\mathrm{Su}_{1}\right]\right)$ we will refer to $\left[\mathrm{TV}_{2}\right.$, Section 2].

We formulate in 3.2 our basic deformation statement $\mathcal{D}(X ; \mathcal{Y})$ in the generality of deforming embeddings on an arbitrary locally compact, locally connected metric space $X$ respectfully to the members of quite an arbitrary family $\mathcal{Y}$ of subsets of $X$. This generality is possible because of the use of LQS (= locally quasisymmetric) embeddings in place of LQC embeddings. Quasisymmetric embeddings of metric spaces were introduced in $\left[\mathrm{TV}_{1}\right]$. In Euclidean spaces of dimension at least two, quasisymmetry is closely related to quasiconformality. This relationship will be used in proofs in both directions. To avoid technical difficulties connected with one-dimensional LQS embeddings, we study this case more closely in a separate paper [Lu], to which reference will be made in some proofs.

We keep track of the bi-Lipschitz constants, the quasisymmetry parameters, and the dilatations of the embeddings, and obtain quantitative versions of results of [SS].

The paper is divided into eight sections. In the preliminary second section we prove, in particular, a canonical Schoenflies theorem. In Section 3 we introduce a deformation statement $\mathcal{D}(X ; \mathcal{Y})$, as said above, and prove elementary lemmas related to it. In Sections 4 and 5 we establish the statements $\mathcal{D}\left(\mathbf{R}^{n} ; \mathcal{Y}_{n}\right)$ and $\mathcal{D}\left(\mathbf{R}_{+}^{n} ; \mathcal{Y}_{n}^{+}\right)$. Section 4 is devoted to proving an inductive handle lemma for $\mathbf{R}^{n}$ and another one for $\mathbf{R}_{+}^{n}$. In Section 6 we first apply the previously established property of $\mathbf{R}^{n}$ and of $\mathbf{R}_{+}^{n}$ to strengthen it in Theorem 6.2, which is the main result of this paper. Theorem 6.6 is a substitute of this theorem for the majorant topology. In Section 7 we consider deformation of embeddings of LIP manifolds and of LQS manifolds. Section 8 gives elementary applications to local contractibility of groups of CAT homeomorphisms, to CAT isotopies, and to counting compact CAT manifolds for CAT = LIP or LQS, all known in the context of topological 
manifolds and partially known for CAT $=$ LIP .

In a paper in preparation I shall apply results of the present paper to a study of locally LIP (or LQS) flat embeddings of codimension at least three.

\section{Preliminaries}

2.1. Notation and terminology. For an integer $n \geq 0$, we let $\mathbf{R}^{n}$ be the Euclidean $n$-space and $\mathbf{R}_{+}^{n}=\left\{x \in \mathbf{R}^{n} \mid x_{n} \geq 0\right\}$ with $\mathbf{R}_{+}^{0}=\mathbf{R}^{0}$. Letting $\mathcal{Y}_{1}$ be the family of the subsets $\mathbf{R}^{1}, \mathbf{R}_{+}^{1}, \mathbf{R}_{-}^{1}=(-\infty, 0]$, and $\{0\}$ of $\mathbf{R}^{1}$, we define

$$
\mathcal{Y}_{n}=\left\{Y_{1} \times \cdots \times Y_{n} \subset \mathbf{R}^{n} \mid Y_{i} \in \mathcal{Y}_{1}, \quad i=1, \ldots, n\right\}
$$

and $\mathcal{Y}_{n}^{+}=\left\{Y \in \mathcal{Y}_{n} \mid Y \subset \mathbf{R}_{+}^{n}\right\}$ for $n \geq 1$. We identify $\mathbf{R}^{m}, m<n$, with the subspace $\mathbf{R}^{m} \times 0$ of $\mathbf{R}^{n}=\mathbf{R}^{m} \times \mathbf{R}^{n-m}$ if not otherwise stated. For $n \geq 0$ and $r>0$ we set $I^{n}=[-1,1]^{n}, I^{n}(r)=r I^{n}, J^{n}=(-1,1)^{n}, J_{+}^{n}=J^{n} \cap \mathbf{R}_{+}^{n}$, $J^{n}(r)=r J^{n}, B^{n}(a, r)=\left\{x \in \mathbf{R}^{n}|| x-a \mid<r\right\}$ if $a \in \mathbf{R}^{n}, B^{n}=B^{n}(0,1)$, $B^{n}(r)=r B^{n}$, and $S^{n-1}=\partial B^{n}$. Let $I=[0,1]$.

If $X$ is a topological space and $A, B \subset X$, we denote the interior of $A$ by $\operatorname{int} A$ and the inclusion $\bar{A} \subset \operatorname{int} B$ by $A \subset \subset B$. If $M$ is a manifold, $\partial M$ denotes the boundary and Int $M$ the interior of $M$. If not otherwise stated, we denote every metric (occasionally also Euclidean ones) by $d$ and metrize the Cartesian product of finitely many metric spaces (except for the factorizations $\mathbf{R}^{n}=\mathbf{R}^{m} \times \mathbf{R}^{n-m}$ ) by the usual maximum metric. Let $(X, d)$ be a metric space. If $a \in X$ and $r>0$, we let $B_{d}(a, r)$ denote the open ball $\{x \in X \mid d(x, a)<r\}$. If $A, B \subset X$, we let $d(A, B)$ denote the distance between $A$ and $B$ (with $d(x, B)=d(\{x\}, B))$ and $d(A)$ the diameter of $A$.

If $A \subset B$, we let id denote the inclusion map $A \rightarrow B$.

Let $X$ be a set and $U, Y \subset X$. An injection $f: U \rightarrow X$ is said to respect $Y$ if $f^{-1} Y=U \cap Y$ or, equivalently, if $f[U \cap Y]=f U \cap Y$.

Suppose that $X$ and $Y$ are topological spaces. Let $C(X ; Y)$ denote the set of all continuous maps of $X$ into $Y$. We equip $C(X ; Y)$ and its subsets with the compact-open topology if not otherwise stated. Suppose that $(Y, d)$ is a metric space. If $f, g: X \rightarrow Y$ and $A \subset X$, we write

$$
d(f, g ; A)=\sup \{d(f(x), g(x)) \mid x \in A\}
$$

and $d(f, g)=d(f, g ; X)$. In the majorant topology of $C(X ; Y)$ an open neighbourhood basis of $f \in C(X ; Y)$ is given by the sets

$$
N_{\varepsilon}(f)=\{g \in C(X ; Y) \mid d(f(x), g(x))<\varepsilon(x) \text { for all } x \in X\}
$$

where $\varepsilon \in C(X ;(0, \infty))$, while the sets $N_{\varepsilon}(f)$ with $\varepsilon$ being a positive constant form a neighbourhood basis of $f$ in the uniform topology of $C(X ; Y)$. If $X$ is metrizable, the majorant topology is independent of the metric of $Y$. 
If $X$ is a topological space and if $U$ and $A$ are subsets of $X$ with $U$ open, let $E(U, A ; X)$ denote the set of all open embeddings of $U$ into $X$ which are the identity on $U \cap A$, and let $H(X, A)$ denote the group of all homeomorphisms of $X$ onto itself which are the identity on $A$. Let $E(U ; X)=E(U, \emptyset ; X)$ and $H(X)=H(X, \emptyset)$. Note that a continuous injection of an open subset of a manifold $X$ into $X$ is open if and only if it respects $\partial X$.

Let $X$ and $\Lambda$ be topological spaces, $U \subset X$ open, and $f: U \times \Lambda \rightarrow X \times \Lambda$ a function of the form $(x, \lambda) \mapsto\left(f_{\lambda}(x), \lambda\right)$. If $f$, also denoted by $\left(f_{\lambda}\right)_{\lambda \in \Lambda}$, is an open embedding, $f$ is called a $\Lambda$-isotopy (or an isotopy parametrized by $\Lambda$ ) of $U$ into $X$. We let $I_{\Lambda}(U ; X)$ denote the subspace of $E(U \times \Lambda ; X \times \Lambda)$ consisting of all $\Lambda$-isotopies. Let $I_{\Lambda}(X)$ denote the space of all homeomorphic $\Lambda$-isotopies of $X$ onto $X$. In the case $\Lambda=I$, the prefix $\Lambda$ and the subscript $\Lambda$ will be omitted. Note that if $\Lambda$ is a one-point space, we can identify $X \times \Lambda$ with $X$ and $I_{\Lambda}(U ; X)$ with $E(U ; X)$. Now suppose that $X$ is a locally compact Hausdorff space and that $X$ or $\Lambda$ is locally connected. Then, by [Si, 1.6], a function $f$ as above is a $\Lambda$-isotopy if and only if $f_{\lambda} \in E(U ; X)$ for each $\lambda \in \Lambda$ and $f$ is continuous or, equivalently ([Du, XII.3.1]), the function $\lambda \mapsto f_{\lambda}$ of $\Lambda$ into $E(U ; X)$ is continuous.

Let $X$ and $Y$ be metric spaces and $f: X \rightarrow Y$ an embedding. If there is $L \geq 1$ such that

$$
d(x, y) / L \leq d(f(x), f(y)) \leq L d(x, y) \quad \text { for all } x, y \in X,
$$

then $f$ is bi-Lipschitz (abbreviated BL). We also say that $f$ is $L-\mathrm{BL}$. As soon as $f$ satisfies the right-hand inequality, $f$ is called $L$-Lipschitz. If there is $\eta \in H\left(\mathbf{R}_{+}^{1}\right)$ such that

$$
\frac{d(f(a), f(x))}{d(f(b), f(x))} \leq \eta\left(\frac{d(a, x)}{d(b, x)}\right) \text { for } a, b, x \in X, b \neq x,
$$

then $f$ is quasisymmetric (abbreviated QS). We also say that $f$ is $\eta-\mathrm{QS}$. The basic theory of QS embeddings is given in $\left[\mathrm{TV}_{1}\right]$ and [Vä그. If $f$ is $L-\mathrm{BL}, f$ is $\eta$-QS with $\eta(t)=L^{2} t$. Like BL embeddings, QS embeddings form a category, and if $f$ is an $\eta$-QS homeomorphism, $f^{-1}$ is $\eta^{\prime}$-QS with $\eta^{\prime}(t)=\eta^{-1}\left(t^{-1}\right)^{-1}$ for $t>0$. We say that $f$ is, respectively, locally $L$-Lipschitz for $L \geq 1$, LIP, locally $L$-BL for $L \geq 1$, LQS, or locally $\eta$-QS for $\eta \in H\left(\mathbf{R}_{+}^{1}\right)$ if each point of $X$ has a neighbourhood on which $f$ is, respectively, $L$-Lipschitz, BL, $L$-BL, QS, or $\eta-$ QS. (In [LV] 'LIP' refers to locally Lipschitz.) If $X$ is compact and $f$ is LIP or LQS, then $f$ is $\mathrm{BL}$ or $\mathrm{QS}\left[\mathrm{TV}_{1}, 2.23\right]$, respectively. A map $g: X \rightarrow Y$ is called a LIP immersion if each point of $X$ has a neighbourhood on which $g$ is a LIP embedding.

2.2. Quasisymmetry and quasiconformality. Let $Y \in \mathcal{Y}_{n}$, let $p=\operatorname{dim} Y \geq 2$, let $U$ be an open subset of $Y$, and let $f \in E(U ; Y)$. If there is $K \geq 1$ such that for each component $G$ of $\operatorname{Int} U$ the embedding $G \rightarrow \operatorname{Int} Y$ defined by $f$ is 
$K$-quasiconformal in the sense of [V $\left.\ddot{a}_{1}\right], f$ is said to be $K$-QC. If $f$ is locally $\eta$ $\mathrm{QS}$, this is the case with $K=\eta(1)^{p-1}$ by [Vä, 34.2 ]. Conversely, if $f$ is $K-\mathrm{QC}$, Lemma 2.3 below implies that $f$ is locally $\eta$-QS with $\eta$ depending only on $K$ and $p$ (in fact, if $Y$ is affinely isomorphic with $\mathbf{R}^{p}$ or $\mathbf{R}_{+}^{p}$, we can choose $\eta$ to depend only on $K$ by [AVV, 5.23] and [V $\left.\ddot{a}_{1}, 35.2\right]$ ).

It follows that the main results of Sections 5 and 6 for locally $\eta$-QS embeddings hold equally well for $K-\mathrm{QC}$ embeddings. In fact, these results for QC embeddings could be obtained without using QS (or locally $\eta-\mathrm{QS}$ ) embeddings at all; see 5.9.

2.3. Lemma. Let $Y \in \mathcal{Y}_{n}$ and $p=\operatorname{dim} Y \geq 2$. Suppose that either $G$ is a connected open subset of $Y$ and $F$ a compact subset of $G$ or $F=G=Y$, and let $f: G \rightarrow Y$ be an open $K-\mathrm{QC}$ embedding. Then $f \mid F$ is $\eta-\mathrm{QS}$ with $\eta$ depending only on $G, F$, and $K$.

Proof. The case $\partial Y=\emptyset$ is [V $\ddot{V}_{2}, 2.7$ and 2.4]. Suppose $\partial Y \neq \emptyset$. Then there is a BL homeomorphism $Y \rightarrow \mathbf{R}_{+}^{p}$. Thus, we may assume that $Y=\mathbf{R}_{+}^{p}$. Then [V $\left.\ddot{a}_{1}, 35.2\right]$ reduces the claim to the case $Y=\mathbf{R}^{p}$ considered first. $\square$

The next two lemmas deal with piecewise definability of the BL or QS property for embeddings close to the identity. A number $\gamma>0$ is called a Lebesgue number of a cover $\mathcal{A}$ of a metric space $X$ if $B \subset X$ and $d(B)<\gamma$ imply that $B \subset A$ for some $A \in \mathcal{A}$.

2.4. Lemma. Let $X$ be a metric space, let $A \subset X$, let $\left(A_{\beta}\right)$ be a cover of the space $A$ having a Lebesgue number $\gamma>0$, and let $L \geq 1$. If $f: A \rightarrow X$ is an embedding such that $f \mid A_{\beta}$ is $L-\mathrm{BL}$ for each $\beta$ and $d(f, \mathrm{id}) \leq \frac{1}{4} \gamma$, then $f$ is $L_{1}-\mathrm{BL}$ with $L_{1}=\max (L, 2)$.

Proof. Let $x, y \in A$. Then $|d(f(x), f(y))-d(x, y)| \leq \frac{1}{2} \gamma \leq \frac{1}{2} d(x, y)$ if $d(x, y) \geq \gamma$, and $\{x, y\} \subset A_{\beta}$ for some $\beta$ if $d(x, y)<\gamma$. व

2.5. Lemma. Let $X, A,\left(A_{\beta}\right), \gamma$ be as in 2.4 with $A$ bounded and such that $\gamma<d\left(A_{\beta}\right)$ whenever $d\left(A_{\beta}\right)>0$. Let $c \geq d(A) / \gamma$. Then for each $\eta \in H\left(\mathbf{R}_{+}^{1}\right)$ there is $\eta_{1} \in H\left(\mathbf{R}_{+}^{1}\right)$ depending only on $\eta$ and $c$ with the following property: If $f: A \rightarrow X$ is an embedding such that $f \mid A_{\beta}$ is $\eta-Q S$ for each $\beta$ and $d(f, \mathrm{id}) \leq \frac{1}{8} \gamma$, then $f$ is $\eta_{1}-\mathrm{QS}$.

Proof. Since $d(f(x), f(y)) \geq \frac{1}{4} \gamma$ for $x, y \in A$ with $d(x, y) \geq \frac{1}{2} \gamma$ and since $d(f A) \leq c \gamma+\frac{1}{4} \gamma$, the proof of $\left[\mathrm{TV}_{1}, 2.23\right]$ applies. $\square$

In 2.6-2.9 we study the problem of when a BL (or QS) embedding remains $\mathrm{BL}$ (or QS) after a slight BL (or, respectively, QS) perturbation.

2.6. Lemma. Let $X$ be a metric space, let $A \subset V \subset U \subset X$, and let $f, g: U \rightarrow X$ be embeddings such that $f$ and $g \mid V$ are $L-\mathrm{BL}, f=g$ on $U \backslash A$, and $d(f, g) \leq \frac{1}{2} d(f A, f[U \backslash V])$. Then $g$ is $2 L-\mathrm{BL}$. 
2.7. Corollary. Let $X$ be a locally compact, locally connected metric space, and let $A \subset V \subset U \subset X$ with $A$ compact and $U, V$ open. Then there is $\varepsilon>0$ with the following property: Suppose that $\Lambda$ is a metric space, that $f, g \in$ $I_{\Lambda}(U ; X)$, that $f=g$ on $(U \backslash A) \times \Lambda$, that $d(h$,id; $V \times \Lambda) \leq \varepsilon$ if $h=f$ or $g$, that $Y \subset X$, and that $f \mid(U \cap Y) \times \Lambda$ and $g \mid(V \cap Y) \times \Lambda$ are $L$-BL. Then $g \mid(U \cap Y) \times \Lambda$ is $2 L-\mathrm{BL}$.

Proof. Choose open sets $V_{1}, V_{2} \subset X$ such that $A \subset V_{2} \subset \subset V_{1} \subset \subset V$ with $\bar{V}_{1}$ compact. Choose $\gamma>0$ with $\gamma \leq d\left(\bar{V}_{2}, X \backslash V_{1}\right)$. By [Si, 1.7] there is $\varepsilon \in\left(0, \frac{1}{4} \gamma\right.$ ] such that if $h \in E(V ; X)$ and $d(h$, id $) \leq \varepsilon$, then $h A \subset V_{2}$ and $h V \supset \bar{V}_{1}$. If now $f$ and $g$ are as in the corollary, then $d(f, g) \leq \frac{1}{2} \gamma$ and $d(f[A \times \Lambda], f[(U \backslash V) \times \Lambda]) \geq \gamma$. Thus, $\varepsilon$ is the desired number by 2.6 . $\square$

2.8. Lemma. Let $X, A, U, V$ be as in 2.6 with $V$ bounded, let $\gamma>0$ be such that $\gamma \leq d(A, U \backslash V)$ and such that $\gamma<\frac{1}{2} d(V)$ if $d(V)>0$, let $c \geq d(V) / \gamma$, and let $f, g: U \rightarrow X$ be embeddings such that $f$ and $g \mid V$ are $\eta$-QS, that $f=g$ on $U \backslash A$, that $d(f A, f[U \backslash V]) \geq \gamma$, and that $d(h$,id; $V)<\frac{1}{7} \gamma$ if $h=f$ or $g$. Then $g$ is $\eta_{1}-\mathrm{QS}$ with $\eta_{1}$ depending only on $\eta$ and $c$.

Proof. As shown in [LT, p. 356], there is $\bar{\eta} \in H\left(\mathbf{R}_{+}^{1}\right)$ depending only on $\eta$ such that $\eta(s) \eta(t) \leq \bar{\eta}(s t)$ for all $s, t \in I$. Let $a, b, x$ be distinct points in $U$, and let

$$
\varrho=\frac{d(a, x)}{d(b, x)} \quad \text { and } \quad \varrho_{1}=\frac{d(g(a), g(x))}{d(g(b), g(x))} .
$$

We must find $\eta_{1} \in H\left(\mathbf{R}_{+}^{1}\right)$ depending only on $(\eta, c)$ such that $\varrho_{1} \leq \eta_{1}(\varrho)$. We may assume that $\{a, b, x\}$ meets both $A$ and $U \backslash V$ as $\varrho_{1} \leq \eta(\varrho)$ in the contrary case. We divide the consideration into eight disjoint cases such that $x \in A$ in Cases 1-3, $x \in U \backslash V$ in Cases 4-6, and $x \in V \backslash A$ in Cases 7-8. Let $\varepsilon=\frac{2}{7} \gamma$ and $\varrho_{0}=d(f(a), f(x)) / d(f(b), f(x))$. By $c_{1}, \ldots, c_{8}$ we denote absolute positive constants. we get

Case 1: $x \in A, a, b \in U \backslash V$. Since $d(f(a), f(x)) \geq \gamma$ and $d(f(b), f(x)) \geq \gamma$,

$$
\varrho_{1} \leq \frac{d(f(a), f(x))+\varepsilon}{d(f(b), f(x))-\varepsilon} \leq c_{1} \varrho_{0} \leq c_{1} \eta(\varrho) .
$$

Case 2: $x \in A, a \in U \backslash V, b \in V$. Choose $y \in V$ with $d(y, x) \geq$ $\max (\gamma, d(b, x))$. Then $d(f(y), f(x)) \geq \gamma-\varepsilon$. Hence

$$
\begin{aligned}
\varrho_{1} & =\frac{d(g(a), g(x))}{d(g(y), g(x))} \cdot \frac{d(g(y), g(x))}{d(g(b), g(x))} \leq c_{2} \frac{d(f(a), f(x))}{d(f(y), f(x))} \eta\left(\frac{d(y, x)}{d(b, x)}\right) \\
& \leq c_{2} \eta\left(\frac{d(a, x)}{d(y, x)}\right) \eta\left(\frac{d(y, x)}{d(a, x)} \varrho\right) \leq c_{2} \eta(\varrho) \eta(c \varrho) .
\end{aligned}
$$


Case 3: $x \in A, a \in V, b \in U \backslash V$. Choose $y \in V$ with $d(y, x) \geq$ $\max (\gamma, d(a, x))$. Then

$$
\begin{aligned}
\varrho_{1} & =\frac{d(g(a), g(x))}{d(g(y), g(x))} \cdot \frac{d(g(y), g(x))}{d(f(b), g(x))} \leq \eta\left(\frac{d(a, x)}{d(y, x)}\right) c_{3} \frac{d(f(y), f(x))}{d(f(b), f(x))} \\
& \leq c_{3} \eta\left(\frac{d(a, x)}{d(y, x)}\right) \eta\left(\frac{d(y, x)}{d(b, x)}\right) .
\end{aligned}
$$

Subcase 3.1: $d(y, x) \leq d(b, x)$. Now $\varrho_{1} \leq c_{3} \bar{\eta}(\varrho)$.

Subcase 3.2: $d(y, x) \geq d(b, x)$. Now $\varrho_{1} \leq c_{3} \eta(\varrho) \eta(c)$.

Case 4: $x \in U \backslash V, a, b \in A$. As in Case 1, we get

$$
\varrho_{1}=\frac{d(g(a), f(x))}{d(g(b), f(x))} \leq c_{4} \varrho_{0} \leq c_{4} \eta(\varrho) .
$$

Case 5: $x \in U \backslash V, a \in A, b \in U \backslash A$. Now

$$
\varrho_{1}=\frac{d(g(a), f(x))}{d(f(b), f(x))} \leq c_{5} \varrho_{0} \leq c_{5} \eta(\varrho) .
$$

Case 6: $x \in U \backslash V, a \in U \backslash A, b \in A$. Now

$$
\varrho_{1}=\frac{d(f(a), f(x))}{d(g(b), f(x))} \leq c_{6} \varrho_{0} \leq c_{6} \eta(\varrho) .
$$

Case 7: $x \in V \backslash A, a \in A, b \in U \backslash V$. Choose $y \in V$ as in Case 3. Then

$$
\varrho_{1}=\frac{d(g(a), g(x))}{d(g(y), g(x))} \cdot \frac{d(g(y), f(x))}{d(f(b), f(x))} \leq c_{7} \eta\left(\frac{d(a, x)}{d(y, x)}\right) \eta\left(\frac{d(y, x)}{d(b, x)}\right) .
$$

Subcase 7.1: $d(y, x) \leq d(b, x)$. Now $\varrho_{1} \leq c_{7} \bar{\eta}(\varrho)$.

Subcase 7.2: $d(y, x) \geq d(b, x)$. Since $\gamma \leq d(a, b) \leq d(a, x)+d(b, x)$, we have that $d(a, x) \geq \frac{1}{2} \gamma$ or $d(b, x) \geq \frac{1}{2} \gamma$.

Subsubcase 7.2.1: $d(a, x) \geq \frac{1}{2} \gamma$. Now $\varrho_{1} \leq c_{7} \eta(\varrho) \eta(2 c \varrho)$.

Subsubcase 7.2.2: $d(b, x) \geq \frac{1}{2} \gamma$. Now $\varrho_{1} \leq c_{7} \eta(\varrho) \eta(2 c)$.

Case 8: $x \in V \backslash A, a \in U \backslash V, b \in A$. Choose $y \in V$ as in Case 2. Then

$$
\varrho_{1}=\frac{d(f(a), f(x))}{d(g(y), f(x))} \cdot \frac{d(g(y), g(x))}{d(g(b), g(x))} \leq c_{8} \eta\left(\frac{d(a, x)}{d(y, x)}\right) \eta\left(\frac{d(y, x)}{d(b, x)}\right) .
$$

As in Case 7.2, we have two subcases:

Subcase 8.1: $d(a, x) \geq \frac{1}{2} \gamma$. Now $\varrho_{1} \leq c_{8} \eta(\varrho) \eta(2 c \varrho)$.

Subcase 8.2: $d(b, x) \geq \frac{1}{2} \gamma$. Now $\varrho_{1} \leq c_{8} \eta(\varrho) \eta(2 c)$.

Thus, we can construct $\eta_{1}$ depending only on $(\eta, c)$ such that always $\varrho_{1} \leq$ $\eta_{1}(\varrho)$. 口 
2.9. Corollary. Let $X, A, U, V$ be as in 2.7 with $V$ bounded, and let $\gamma_{0}>0$. Then there is $\varepsilon>0$ such that for each $\eta \in H\left(\mathbf{R}_{+}^{1}\right)$ there is $\eta_{1} \in H\left(\mathbf{R}_{+}^{1}\right)$ with the following property: Suppose that $f, g \in E(U ; X)$, that $f=g$ on $U \backslash A$, that $d(h$, id; $V) \leq \varepsilon$ if $h=f$ or $g$, that $Y \subset X$ with $d(V \cap Y) \notin\left(0, \gamma_{0}\right)$, and that $f \mid U \cap Y$ and $g \mid V \cap Y$ are $\eta$-QS. Then $g \mid U \cap Y$ is $\eta_{1}-\mathrm{QS}$.

Proof. If $\gamma$ and $\varepsilon$ are chosen as in the proof of 2.7 with $\gamma<\frac{1}{2} \gamma_{0}$ and $\varepsilon<\frac{1}{7} \gamma$, then $\varepsilon$ is the desired number by 2.8 . 口

The next lemma deals with composition of $\mathrm{BL}$ isotopies.

2.10. Lemma. Let $U, X$, and $\Lambda$ be metric spaces, let $a=t_{0}<t_{1}<\cdots<$ $t_{n}=b$ be real numbers, let $L \geq 1$, and let $f: U \times \Lambda \times[a, b] \rightarrow X \times \Lambda \times[a, b]$ be an embedding of the form $(x, \lambda, t) \mapsto\left(f_{\lambda t}(x), \lambda, t\right)$ such that $f_{\lambda t} U=f_{\lambda a} U$ for each $(\lambda, t) \in \Lambda \times[a, b]$ and such that $f \mid U \times \Lambda \times\left[t_{i-1}, t_{i}\right]$ is $L-\mathrm{BL}$ for $1 \leq i \leq n$. Then $f$ is $2 L-\mathrm{BL}$.

Proof. Consider two points $y=(x, \lambda, t)$ and $y^{\prime}=\left(x^{\prime}, \lambda^{\prime}, t^{\prime}\right)$ in $A=f[U \times$ $\Lambda \times[a, b]]$ with $t_{i-1} \leq t<t_{i} \leq t_{j-1}<t^{\prime} \leq t_{j}$ for some $i, j, i<j$. Then the points $z_{i-1}=\left(x^{\prime}, \lambda^{\prime}, t\right), z_{k}=\left(x^{\prime}, \lambda^{\prime}, t_{k}\right)$ for $i \leq k \leq j-1$, and $z_{j}=y^{\prime}$ are in $A$, whence

$$
\begin{aligned}
d\left(f^{-1}(y), f^{-1}\left(y^{\prime}\right)\right) & \leq d\left(f^{-1}(y), f^{-1}\left(z_{i-1}\right)\right)+\sum_{k=i}^{j} d\left(f^{-1}\left(z_{k-1}\right), f^{-1}\left(z_{k}\right)\right) \\
& \leq L \max \left(d\left(x, x^{\prime}\right), d\left(\lambda, \lambda^{\prime}\right)\right)+L\left|t-t^{\prime}\right| \leq 2 L d\left(y, y^{\prime}\right) .
\end{aligned}
$$

Thus, $f^{-1}$ is $2 L$-Lipschitz. Similarly it is shown that $f$ is $2 L$-Lipschitz. व

The following simple fact about function spaces will be used often.

2.11. Lemma. Let $X$ be a metric space, let $A, B \subset X$ be compact, let $E_{A}$ and $E_{B}$ be the spaces of embeddings of $A$ or $B$, respectively, into $X$, and let $T=\left\{(f, g) \in E_{A} \times E_{B} \mid f A \subset g B\right\}$. Then the function $T \rightarrow E_{A},(f, g) \mapsto g^{-1} f$, is continuous.

Proof. Fix $\left(f_{0}, g_{0}\right) \in T$ and $\varepsilon>0$. Since $g_{0}^{-1}: g_{0} B \rightarrow B$ is uniformly continuous, there is $\delta>0$ such that if $x, y \in B$ and $d\left(g_{0}(x), g_{0}(y)\right)<2 \delta$, then $d(x, y)<\varepsilon$. Now let $(f, g) \in T$ with $d\left(f, f_{0}\right)<\delta$ and $d\left(g, g_{0}\right)<\delta$. Then

$$
\begin{aligned}
d\left(g_{0} g^{-1} f, f_{0} ; A\right) & =d\left(g_{0} g^{-1}, f_{0} f^{-1} ; f A\right) \leq d\left(g_{0} g^{-1}, \mathrm{id} ; f A\right)+d\left(\mathrm{id}, f_{0} f^{-1} ; f A\right) \\
& \leq d\left(g_{0} g^{-1}, \mathrm{id} ; g B\right)+d\left(f, f_{0} ; A\right) \leq d\left(g_{0}, g ; B\right)+d\left(f, f_{0} ; A\right)<2 \delta
\end{aligned}
$$

whence $d\left(g^{-1} f, g_{0}^{-1} f_{0} ; A\right)<\varepsilon$. ᄆ 
2.12. We next improve in certain respects the quantitative canonical Schoenflies theorem for embeddings near id given in $\left[\mathrm{TV}_{2}, 3.2\right]$. The construction of the extension operator is essentially the same as in the proofs of $\left[\mathrm{TV}_{2}, 3.2\right]$ and of [GV, Lemma 9] and, thus, uses the furling idea of M. Brown. Our proof is detailed except for the tedious verification of the preservation of the quasisymmetry property in dimension one, for which we refer to [Lu]. Recall that each element of $\mathcal{Y}_{n}$ is a closed convex cone in $\mathbf{R}^{n}$ with vertex 0 .

2.13. Theorem. Let $n \geq 1$, let $(X, \mathcal{Y})=\left(\mathbf{R}^{n}, \mathcal{Y}_{n}\right)$ or $\left(\mathbf{R}_{+}^{n}, \mathcal{Y}_{n}^{+}\right)$, and let $A=\left(B^{n} \backslash \bar{B}^{n}\left(\frac{1}{3}\right)\right) \cap X$. Then there exist a neighbourhood $\mathcal{P}$ of id: $A \rightarrow X$ in $E(A ; X)$ and a continuous map $\varphi: \mathcal{P} \rightarrow E\left(B^{n} \cap X ; X\right)$ having the following properties:

(1) $\varphi($ id $)=$ id .

(2) $\varphi(h)=h$ on $\left(B^{n} \backslash B^{n}\left(\frac{2}{3}\right)\right) \cap X$ for each $h \in \mathcal{P}$.

(3) If $h \in \mathcal{P}$ respects $Y \in \mathcal{Y}$, so does $\varphi(h)$. If $h, h^{\prime} \in \mathcal{P}$ respect $Y \in \mathcal{Y}$ and $h=h^{\prime}$ on $A \cap Y$, then $\varphi(h)=\varphi\left(h^{\prime}\right)$ on $B^{n} \cap Y$.

(4) Let $\Lambda$ be a metric space, and let $f=\left(f_{\lambda}\right)_{\lambda \in \Lambda} \in I_{\Lambda}(A ; X)$ with $f_{\lambda} \in \mathcal{P}$ for each $\lambda \in \Lambda$. Define $\varphi(f)=\left(\varphi\left(f_{\lambda}\right)\right)_{\lambda \in \Lambda} \in I_{\Lambda}\left(B^{n} \cap X ; X\right)$. Let $Y \in \mathcal{Y}$, and suppose that $f$ respects $Y \times \Lambda$ and that $f \mid(A \cap Y) \times \Lambda \in I_{\Lambda}(A \cap Y ; Y)$ is (a) LIP or (b) locally $L$-BL or (c) $L$-BL. Then $\varphi(f) \mid\left(B^{n} \cap Y\right) \times \Lambda \in I_{\Lambda}\left(B^{n} \cap Y ; Y\right)$ is, respectively, (a) LIP or (b) locally $L^{*}-\mathrm{BL}$ or (c) $L^{*}-\mathrm{BL}$ with $L^{*}$ depending only on $L$.

(5) If $Y \in \mathcal{Y}$, if $p=\operatorname{dim} Y$, if $h \in \mathcal{P}$ respects $Y$, and if $h \mid A \cap Y \in$ $E(A \cap Y$; $Y$ ) is (a) LQS or (b) $\eta$-QS or, in the case $p \geq 2$, (c) $K-\mathrm{QC}$, then $\varphi(h) \mid B^{n} \cap Y \in E\left(B^{n} \cap Y ; Y\right)$ is, respectively, (a) LQS or (b) (when restricted to $\bar{B}^{n}\left(\frac{3}{4}\right) \cap Y$ whenever $\left.p \geq 2\right) \eta^{*}-\mathrm{QS}$ or (c) $K^{*}-\mathrm{QC}$ with $\eta^{*}$ and $K^{*}$ depending only on $(\eta, p)$ or $(K, p)$, respectively.

Proof. We prove the theorem in the form where (1) is deleted. Then, since $\varphi(\mathrm{id})\left[B^{n} \cap X\right]=B^{n} \cap X$ by (2) and since $\varphi\left(\right.$ id) is $L_{0}$-BL with $L_{0}$ an absolute constant by (4c), replacing $\varphi$ by $\varphi(\cdot) \varphi(\text { id })^{-1}$ yields the full theorem.

For positive reals $a_{1}<a_{2}$ and $b_{1}<b_{2}$, let $\alpha=\alpha_{\left(a_{1}, a_{2} ; b_{1}, b_{2}\right)}: \mathbf{R}_{+}^{1} \rightarrow \mathbf{R}_{+}^{1}$ denote the homeomorphism that maps $\left[0, a_{1}\right]$ and $\left[a_{1}, a_{2}\right]$ affinely onto $\left[0, b_{1}\right]$ or $\left[b_{1}, b_{2}\right]$, respectively, and is a translation on $\left[a_{2}, \infty\right)$. Then define a selfhomeomorphism $\alpha_{n}=\alpha_{\left(a_{1}, a_{2} ; b_{1}, b_{2} ; n\right)}$ of $\mathbf{R}^{n}$ setting $\alpha_{n}(x)=\alpha(|x|) x /|x|$ for $x \neq 0$. Fix real numbers $\frac{1}{2}<a<e<c=\frac{2}{3}<b<q<r<1$ independently of $n$ with $b / a=2 c=\frac{4}{3}=\varrho$ and with $r \geq \frac{3}{4}$. Then let $\kappa, \mu, \nu: \mathbf{R}^{n} \rightarrow \mathbf{R}^{n}$ be the homeomorphisms

$$
\kappa=\alpha_{(1 / 2, c ; c, r ; n)}, \quad \mu=\alpha_{(b, q ; a, q ; n)}, \quad \nu=\alpha_{(e, r ; e, c ; n)} .
$$

For reals $0 \leq a_{1} \leq a_{2}$, let $\left[a_{1}, a_{2}\right]=\left\{x \in X\left|a_{1} \leq\right| x \mid \leq a_{2}\right\}$ with $\left[a_{1}\right]=\left[a_{1}, a_{1}\right]$ and with obvious analogous meanings for $\left(a_{1}, a_{2}\right)$ and $\left[a_{1}, a_{2}\right)$. We 
choose a neighbourhood $\mathcal{P}$ of id in $E(A ; X)$ so small that for each $h \in \mathcal{P}$,

$$
h\left[\frac{1}{2}\right] \subset[0, a), \quad[a] \subset h\left(\frac{1}{2}, e\right), \quad \text { and } \quad[b, q] \subset h(c, r) .
$$
by

Consider $h \in \mathcal{P}$. Define an embedding $\bar{h}:\left[\frac{1}{2}, 1\right) \rightarrow X$, which respects $\partial X$,

$$
\bar{h}(x)= \begin{cases}h(x) & \text { if }|x| \geq c \\ h \nu h^{-1} \mu h \kappa(x) & \text { if }|x| \leq c \text { and } \mu h \kappa(x) \in h[e, r] \\ \mu h \kappa(x) & \text { if }|x| \leq c \text { and } \mu h \kappa(x) \in h\left[\frac{1}{2}, e\right] \cup[0, a] .\end{cases}
$$

We show that $\bar{h}$ is well-defined. Let $x \in\left[\frac{1}{2}, c\right]$. Then $\kappa(x) \in[c, r]$, whence $y=h \kappa(x)$ is defined. Let $z=\mu h \kappa(x)$. To see that $\bar{h}(x)$ is defined in at least one way, note that if $|y| \geq q$, then $z=y \in h[c, r]$; if $y \in[b, q]$, then $z \in[a, q] \subset h\left(\frac{1}{2}, r\right)$; if $|y| \leq b$, then $z \in[0, a]$; and finally, if $z \in h[e, r]$, then $h \nu h^{-1}(z) \in h[e, c]$ is defined. To see that $\bar{h}(x)$ is defined in exactly one way, note that if $|x|=c$, then $z=y \in h[r]$, whence $h \nu h^{-1}(z)=h(x)$; if $z \in h\left[\frac{1}{2}, e\right]$, then $h \nu h^{-1}(z)=z$; and finally, if $z \in[0, a]$, then $z \notin h[e, 1)$. From these considerations it also follows that

$$
\bar{h}(x)=h \nu h^{-1} \mu h \kappa(x) \quad \text { if } x \in\left[\frac{1}{2}, c\right] \text { and } \mu h \kappa(x) \in h\left[\frac{1}{2}, r\right]
$$

and that $\bar{h}$ is injective. By $(2.14), \bar{h} \mid\left[\frac{1}{2}, c\right]$ is continuous as the union of two continuous maps of compact sets. It easily follows that $\bar{h}$ is indeed an embedding. Note that

$$
\bar{h}(x)=\varrho^{-1} \bar{h}(\varrho x) \quad \text { if }|x|=\frac{1}{2} .
$$

Define $\hat{h} \in E\left(B^{n} \cap X ; X\right)$ as follows. Let $\hat{h}=\bar{h}$ on $\left[\frac{1}{2}, 1\right)$, and set $\hat{h}(0)=0$. Suppose $x \in\left(0, \frac{1}{2}\right]$. Then $\varrho^{k} x \in\left[\frac{1}{2}, c\right]$ for one or two $k \in \mathbf{N}$. Let

$$
\hat{h}(x)=\varrho^{-k} \bar{h}\left(\varrho^{k} x\right) .
$$

By (2.16), $\hat{h}$ is well-defined. Define $\varphi(h)=\hat{h}$. Clearly (2) and (3) are satisfied.

To prove the continuity of $\varphi$, choose numbers $s, t$ independently of $n$ such that $\frac{1}{2}<s<t<c$ and $\kappa[t] \subset[0, b)$. Note that $\mu \kappa \mid\left[\frac{1}{2}\right]=$ id. Hence, we can choose $\mathcal{P}$ so small that if $h \in \mathcal{P}$, then $h\left[\frac{1}{2}\right]$ is contained in the bounded component of $X \backslash \mu h \kappa[s]$ and $h \kappa[t] \subset[0, b]$. Since $\mu h \kappa[c]=h[r]$, it follows that $\mu h \kappa[s, c] \subset h\left[\frac{1}{2}, r\right]$. Hence, by (2.15),

$$
\bar{h}=h \nu h^{-1} \mu h \kappa \quad \text { on }[s, c] .
$$

Furthermore, $\mu h \kappa\left[\frac{1}{2}, t\right] \subset[0, a]$, whence by $(2.14)$,

$$
\bar{h}=\varrho^{-1} h \kappa \quad \text { on }\left[\frac{1}{2}, t\right] .
$$


As $d\left(\hat{h}_{1}, \hat{h}_{2} ;[0, c]\right)=d\left(\bar{h}_{1}, \bar{h}_{2} ;\left[\frac{1}{2}, c\right]\right)$ for $h_{1}, h_{2} \in \mathcal{P}$, it follows from (2.18), 2.11, and (2.19) that $\varphi$ is continuous.

For the proof of (4) and (5), define homeomorphisms $\sigma, \tau: \mathbf{R}^{n} \rightarrow \mathbf{R}^{n}$ by $\sigma(x)=\nu \kappa(x)$ if $|x| \leq c, \sigma(x)=x$ otherwise, $\tau(x)=\sigma(\varrho x)$ if $|x| \leq \frac{1}{2}$, and $\tau(x)=\kappa(x)$ otherwise. Then $\kappa, \mu, \nu, \sigma, \tau$ are $a_{0}$-BL with $a_{0} \geq \varrho$ independent of $n$.

Choose a number $u$ independently of $n$ such that $t<u<c$ and $[q] \subset \kappa[0, u)$. We can choose $\mathcal{P}$ so small that $[0, q] \cap h \kappa[u]=\emptyset$ for $h \in \mathcal{P}$. Then, by (2.18),

$$
\bar{h}=h \sigma \quad \text { on }[u, 1) .
$$

Hence, by (2.19) and (2.20),

$$
\hat{h}=\varrho^{-1} h \tau \text { on }[u / \varrho, t] .
$$

Choose numbers $v, w$ independently of $n$ such that $s<v<t<u<w<c$. Let $i=\varphi(\mathrm{id})$. By the continuity of $\varphi$ and [Si, 1.7], we can choose $\mathcal{P}$ so small that for each $h \in \mathcal{P}$,

$$
i[w / \varrho, v] \subset \hat{h}(u / \varrho, t), \quad i[v, w] \subset \bar{h}(s, c), \quad \text { and } \quad i[w, q] \subset \bar{h}(u, r) .
$$

Now let $\Lambda, f, \hat{f}=\varphi(f), Y, L$ be as in (4). To simplify notation (only), we assume that $Y=X$. Let $\mathcal{Z}=\{[u / \varrho, t],[s, c],[u, r]\}$. In the cases (a) and (b), by (2.21), (2.18), (2.20), and the compactness of the sets in $\mathcal{Z}$, for each $\lambda \in \Lambda$ there are an open neighbourhood $\Lambda_{\lambda}$ of $\lambda$ and a number $L_{\lambda} \geq 1$, with $L_{\lambda}=a_{0}^{3} L^{3}=L_{1}$ in (b), such that each $Z \in \mathcal{Z}$ has a finite open cover by sets $U \subset Z$ with $\hat{f} \mid U \times \Lambda_{\lambda}$ being $L_{\lambda}-\mathrm{BL}$. In the case (c), $\hat{f} \mid Z \times \Lambda$ is $L_{1}-\mathrm{BL}$ for each $Z \in \mathcal{Z}$. Obviously, in (a) and (b) it suffices to consider the restrictions $\hat{f} \mid\left(B^{n} \cap X\right) \times \Lambda_{\lambda}(\lambda \in \Lambda)$ of $\hat{f}$ in place of $\hat{f}$. By (2.22) it follows that in (4) we may assume (for some $L_{1} \geq 1$ in the case (a)) that $\hat{f}_{\lambda} \mid\left[\frac{1}{2}, r\right]$ and $\hat{f}_{\lambda}^{-1} \mid i[w / \varrho, q]$ are locally $L_{1}$-Lipschitz whenever $\lambda \in \Lambda$ and that $\hat{f} \mid\{x\} \times \Lambda$ for $x \in\left[\frac{1}{2}, r\right]$ and $\hat{f}^{-1} \mid\{x\} \times \Lambda$ for $x \in i[w / \varrho, q]$ are $L_{1}$-Lipschitz.

For $k \in \mathbf{N}$, we conclude from (2.17) that $\hat{f}_{\lambda} \mid \varrho^{-k}\left[\frac{1}{2}, c\right]$ is locally $L_{1}$-Lipschitz if $\lambda \in \Lambda$ and that $\hat{f} \mid\{x\} \times \Lambda$ is $L_{1}$-Lipschitz if $x \in \varrho^{-k}\left[\frac{1}{2}, c\right]$. Then $\hat{f}_{\lambda} \mid[0, r]$ for $\lambda \in \Lambda$ and $\hat{f} \mid\{x\} \times \Lambda$ for $x \in[0, r]$ are $L_{1}$-Lipschitz. Therefore, $\hat{f} \mid[0, r] \times \Lambda$ is $2 L_{1}$-Lipschitz. On the other hand, if $k \in \mathbf{N}$ and $x \in i\left[\varrho^{-k}[w / \varrho, w]\right]$, then (2.17) and (2.22) imply that $\varrho^{k} x \in i[w / \varrho, w]$ and $\hat{f}_{\lambda}^{-1}(x)=\varrho^{-k} \hat{f}_{\lambda}^{-1}\left(\varrho^{k} x\right)$ for each $\lambda \in \Lambda$. Thus, we obtain as above that $\hat{f}^{-1}$ is $2 L_{1}$-Lipschitz on $i[0, q] \times \Lambda=[0, q] \times \Lambda$. Since $\hat{f}=f$ on $[c, 1) \times \Lambda$ and $\hat{f}^{-1}=f^{-1}$ outside $[0, b) \times \Lambda$, it follows that (4) is satisfied, with $L^{*}=2 L_{1}$ in (b) and $L^{*}=2 L_{1}+L$ (or even $2 L_{1}$ ) in (c).

Let $Y, p \geq 2, h, K$ be as in (5c). Each of $\kappa, \mu, \nu, \sigma, \tau$ is $K_{p}-\mathrm{QC}$ with $K_{p}=a_{0}^{2 p-2}$. By (2.21), (2.18), and (2.20), $\hat{h} \mid(u / \varrho, 1) \cap \dot{Y}$ is $K^{*}-\mathrm{QC}, K^{*}=K_{p}^{3} K^{3}$. 
By (2.17), $\hat{h} \mid(0,1) \cap Y$ is $K^{*}-\mathrm{QC}$. By [V $\left.\ddot{a}_{1}, 17.3\right]$ if $Y$ is a linear subspace of $\mathbf{R}^{n}$ or trivially otherwise, $\hat{h} \mid B^{n} \cap Y$ is $K^{*}-\mathrm{QC}$.

Let $Y, p(\geq 1), h, \eta$ be as in (5a) or (5b). Suppose first that $p \geq 2$. In (b), $h \mid A \cap Y$ is $K-\mathrm{QC}, K=\eta(1)^{p-1}$. Thus, by the above, $\hat{h} \mid B^{n} \cap Y$ is $K^{*}-\mathrm{QC}$ with $K^{*}$ depending only on $(\eta(1), p)$. By $2.3, \hat{h} \mid[0, r] \cap Y$ is $\eta^{*}-\mathrm{QS}$ with $\eta^{*}$ depending only on $\left(K^{*}, p\right)$. In (a), $h$ is QS on a neighbourhood of $\left[\frac{1}{2}, r\right] \cap Y$ in $Y$. Since $\hat{h} \mid[0, r]$ depends on $h \mid\left[\frac{1}{2}, r\right]$ only, it follows, cf. the above, that $\hat{h} \mid[0, r] \cap Y$ is QC. Thus, $\hat{h} \mid B^{n} \cap Y$ is LQS in (a) by 2.2. For the case $p=1$, see [Lu, 2.7 and 2.8]. व

2.23. Remark. If $\mathcal{P}_{0} \subset \mathcal{P}$ is a sufficiently small uniform neighbourhood of the inclusion, by 2.5 the parenthetic restriction in (5b) can be deleted for $h \in \mathcal{P}_{0}$.

The construction of isotopies in the proof of $\mathcal{D}\left(\mathbf{R}^{n} ; \mathcal{Y}_{n}\right)$ will ultimately take place in a situation where the following Alexander's construction applies.

2.24. Lemma. Let $(X, \mathcal{Y})=\left(\mathbf{R}^{n}, \mathcal{Y}_{n}\right)$ or $\left(\mathbf{R}_{+}^{n}, \mathcal{Y}_{n}^{+}\right)$with $n \geq 1$, and let $\mathcal{H}=H\left(X, X \backslash B^{n}\right)$. The function $\psi: \mathcal{H} \times I \rightarrow \mathcal{H},(h, t) \mapsto h_{t}$, defined by $h_{t}(x)=t h(x / t)$ if $0<t \leq 1$ and by $h_{0}=\mathrm{id}$, is continuous and has the following properties:

(1) $\psi(\mathrm{id}, t)=\mathrm{id}$ for each $t \in I$. If $h \in \mathcal{H}$ respects $Y \in \mathcal{Y}$, so does $h_{t}$ for each $t \in I$. If $h, h^{\prime} \in \mathcal{H}$ respect $Y \in \mathcal{Y}$ and $h\left|Y=h^{\prime}\right| Y$, then $h_{t}\left|Y=h_{t}^{\prime}\right| Y$ for each $t \in I$.

(2) Let $\Lambda$ be a metric space, and let $f=\left(f_{\lambda}\right)_{\lambda \in \Lambda} \in I_{\Lambda}(X)$ with $f \mid(X \mid$ $\left.B^{n}\right) \times \Lambda=$ id. Define $\psi^{*}(f)=f^{*}=\left(f_{\lambda t}\right)_{\lambda \in \Lambda, t \in I} \in I_{\Lambda \times I}(X)$. Let $Y \in \mathcal{Y}$, and suppose that $f$ respects $Y \times \Lambda$ and that $f \mid Y \times \Lambda$ is $L-\mathrm{BL}$. Then $f^{*} \mid Y \times \Lambda \times I$ is $(3 L+1)-\mathrm{BL}$.

(3) Suppose that $h \in \mathcal{H}$ respects $Y \in \mathcal{Y}$. If $h \mid Y$ is (a) LQS or (b) locally $\eta$-QS or (c) $\eta$-QS, so is, respectively, $h_{t} \mid Y$ for each $t \in I$.

Proof. The continuity of $\psi$ is well known. The condition (1) is obvious. The condition (3) obtains, because for each $t>0$ the homeomorphism $Y \rightarrow Y$, $x \mapsto t x$, is a similarity. In (2) it suffices to show, by the continuity of $f^{*}$ and since $\left(f^{*}\right)^{-1}=\left(f^{-1}\right)^{*}$, that $f^{*} \mid Y \times \Lambda \times(0,1]$ is $L_{1}$-Lipschitz, $L_{1}=3 L+1$. Thus, consider two points $u=(x, \lambda, s)$ and $v=(y, \mu, t)$ in $Y \times \Lambda \times(0,1]$ with $s \geq t$. Let $\delta=\left|f_{\lambda s}(x)-f_{\lambda t}(x)\right|$. If $|x| \geq s$, then $\delta=|x-x|=0$, whereas if $|x|<s$, then

$$
\begin{aligned}
\delta & \leq\left|s f_{\lambda}(x / s)-t f_{\lambda}(x / s)\right|+t\left|f_{\lambda}(x / s)-f_{\lambda}(x / t)\right| \\
& \leq|s-t|\left|f_{\lambda}(x / s)\right|+t L|s-t||x| / s t \leq(1+L)|s-t| .
\end{aligned}
$$

Since $f_{\lambda t} \mid Y$ is $L-\mathrm{BL}$, this implies that

$$
\left|f_{\lambda s}(x)-f_{\lambda t}(y)\right| \leq(1+L)|s-t|+L|x-y| \leq(2 L+1) d(u, v) .
$$


On the other hand,

$$
\left|f_{\lambda t}(y)-f_{\mu t}(y)\right|=t\left|f_{\lambda}(y / t)-f_{\mu}(y / t)\right| \leq t L d(\lambda, \mu) \leq L d(u, v) .
$$

Hence, $\left|f_{\lambda s}(x)-f_{\mu t}(y)\right| \leq L_{1} d(u, v)$. Thus, $d\left(f^{*}(u), f^{*}(v)\right) \leq L_{1} d(u, v)$. 口

\section{Deformation statements for metric spaces}

3.1. For this section, we fix a locally compact, locally connected metric space $X$ and a family $\mathcal{Y}$ of subsets of $X$ which is weakly locally finite in the sense that each point of $X$ has a neighbourhood $N$ for which the set $\{N \cap Y \mid Y \in \mathcal{Y}\}$ is finite. For example, $X$ might be a locally finite simplicial complex with its barycentric metric and $\mathcal{Y}$ the family of all subcomplexes of $X$ as in [SS, Appendix $\mathrm{B}]$. For the pair $(X, \mathcal{Y})$, we first define three deformation statements, which may or may not hold. We then prove their equivalence, locality, and LIP invariance. These results could also be used in a detailed proof of the deformation theorem in [SS].

Note that if $U \subset X$ is open and $\mathcal{P}$ is a topological space, a function $\varphi: \mathcal{P} \times I \rightarrow$ $E(U ; X),(h, t) \mapsto h_{t}$, is continuous if and only if the function $\mathcal{P} \times I \times U \rightarrow X$, $(h, t, x) \mapsto h_{t}(x)$, is continuous, and in this case $\varphi$ induces a continuous map $\varphi^{*}: \mathcal{P} \rightarrow I(U ; X)$, defined by $\varphi^{*}(h)(x, t)=\left(h_{t}(x), t\right)$. Suppose now that $\mathcal{P}$ is a subspace of $E(U ; X), \varphi$ is continuous, $\Lambda$ is a topological space, and $f=\left(f_{\lambda}\right)_{\lambda \in \Lambda}$ is an isotopy in $I_{\Lambda}(U ; X)$ with $f_{\lambda} \in \mathcal{P}$ for each $\lambda \in \Lambda$. Then we can define an isotopy $\varphi^{*}(f)=\left(f_{\lambda t}\right)_{\lambda \in \Lambda, t \in I}$ in $I_{\Lambda \times I}(U ; X)$.

3.2. Definition. We let $\mathcal{D}(X ; \mathcal{Y})$ denote the following statement:

Let $A$ and $A^{\prime}$ be closed subsets of $X$ with $A \subset \subset A^{\prime}$, let $U$ be an open subset of $X$, and let $B$ and $B^{\prime}$ be compact subsets of $U$ with $B \subset \subset B^{\prime}$. Then the following statement $\mathcal{D}\left(X ; A, A^{\prime}, U, B, B^{\prime} ; \mathcal{Y}\right)$ always holds:

There exist a neighbourhood $\mathcal{P}$ of the inclusion map id: $U \rightarrow X$ in $E\left(U, A^{\prime}\right.$; $X)$ and a continuous map $\varphi: \mathcal{P} \times I \rightarrow E(U, A ; X)$ having the following properties, where $h_{t}=\varphi(h, t)$ for $h \in \mathcal{P}, t \in I$ :

(1) $\varphi(\mathrm{id}, t)=\mathrm{id}$ for each $t \in I$.

(2) $h_{0}=h$ for each $h \in \mathcal{P}$.

(3) $h_{1}=$ id on $B$ for each $h \in \mathcal{P}$.

(4) $h_{t}=h$ on $U \backslash B^{\prime}$ for all $h \in \mathcal{P}, t \in I$.

(5) If $h \in \mathcal{P}$ respects $Y \in \mathcal{Y}$, then $h_{t}$ respects $Y$ for each $t \in I$.

(6) If $h, h^{\prime} \in \mathcal{P}$ respect $Y \in \mathcal{Y}$ and $h=h^{\prime}$ on $U \cap Y$, then $h_{t}=h_{t}^{\prime}$ on $U \cap Y$ for each $t \in I$.

(7) Let $\Lambda$ be a metric space, let $f=\left(f_{\lambda}\right)_{\lambda \in \Lambda} \in I_{\Lambda}(U ; X)$ with $f_{\lambda} \in \mathcal{P}$ for each $\lambda \in \Lambda$, and let $f^{*}=\varphi^{*}(f) \in I_{\Lambda \times I}(U ; X)$. Suppose that $Y \in \mathcal{Y}$, that $f$ respects $Y \times \Lambda$, and that $f \mid(U \cap Y) \times \Lambda \in I_{\Lambda}(U \cap Y ; Y)$ is (a) LIP or (b) locally $L$-BL or (c) $L$-BL. Then $f^{*} \mid(U \cap Y) \times \Lambda \times I \in I_{\Lambda \times I}(U \cap Y ; Y)$ is, respectively, (a) LIP or (b) locally $L^{*}-\mathrm{BL}$ or (c) $L^{*}-\mathrm{BL}$ with $L^{*}$ not depending on $(\Lambda, f)$. 
(8) If $h \in \mathcal{P}$ respects $Y \in \mathcal{Y}$, if $h \mid U \cap Y \in E(U \cap Y$; $Y)$ is (a) LQS or (b) locally $\eta$-QS or (c) $\eta$-QS, and if $t \in I$, then $h_{t} \mid U \cap Y \in E(U \cap Y ; Y)$ is, respectively, (a) LQS or (b) locally $\eta^{*}-\mathrm{QS}$ or (c) $\eta^{*}-\mathrm{QS}$ with $\eta^{*}$ not depending on $(h, t)$.

We let $\mathcal{D}_{Q S}(X ; \mathcal{Y})$ denote $\mathcal{D}(X ; \mathcal{Y})$ with $(7)$ deleted.

3.3. Remark. From (4), (2), and [Si, 1.6.2] it follows that $h_{t} U=h U$ for all $h \in \mathcal{P}, t \in I$. Hence, in (5), $h_{t}[U \cap Y]=h U \cap Y$. If $h \in \mathcal{P}$ respects $Y \in \mathcal{Y}$ and $h \mid U \cap Y=\mathrm{id}$, then (1) and (6) imply that $h_{t} \mid U \cap Y=\mathrm{id}$ for each $t \in I$. If $\mathcal{Q}$ is a neighbourhood of id in $E(U, A ; X)$, it follows from (1) that replacing $\mathcal{P}$ by a sufficiently small neighbourhood of id we may assume that $\varphi[\mathcal{P} \times I] \subset \mathcal{Q}$.

The following concept will be only an auxiliary one.

3.4. Definition. We let $\mathcal{D}_{1}\left(X ; A, A^{\prime}, U, B, B^{\prime} ; \mathcal{Y}\right)$ denote the statement which we obtain from $\mathcal{D}\left(X ; A, A^{\prime}, U, B, B^{\prime} ; \mathcal{Y}\right)$ replacing the compact-open topology of $E\left(U, A^{\prime} ; X\right)$ and of $E(U, A ; X)$ by the uniform topology and supposing that in 3.2(7) the map $\lambda \mapsto f_{\lambda}$ of $\Lambda$ into $\mathcal{P}$ is continuous.

3.5. Remarks. 1 . As in 3.2 , we again have in 3.4 that $\varphi^{*}(h)$ is an isotopy of $U$ onto $h U$ for each $h \in \mathcal{P}$ and that $f^{*} \in I_{\Lambda \times I}(U ; X)$ in 3.2(7). In addition, the map $\varphi^{*}: \mathcal{P} \rightarrow I(U ; X)$ is continuous in the uniform topologies.

2. The older deformation statement in 3.4 implies clearly the newer one, with the same $\mathcal{P}$ and $\varphi$. Conversely, 3.8 below shows that $\mathcal{D}_{1}(X ; \mathcal{Y})$ (as defined in an obvious way) implies $\mathcal{D}(X ; \mathcal{Y})$.

3.6. Definition. We let $\mathcal{D}^{*}(X ; \mathcal{Y})$ denote the following statement:

Let $A, A^{\prime}, U, B, B^{\prime}$ be as in 3.2. Then the following statement $\mathcal{D}^{*}\left(X ; A, A^{\prime}\right.$, $\left.U, B, B^{\prime} ; \mathcal{Y}\right)$ always holds:

There exist a neighbourhood $\mathcal{P}$ of id in $E\left(U, A^{\prime} ; X\right)$ and a continuous map $\psi: \mathcal{P} \times I \rightarrow H(X, A)$ such that if for each $h \in \mathcal{P}$ we define an isotopy $\psi^{*}(h)=$ $\left(h_{t}\right)_{t \in I} \in I(X)$ by $h_{t}=\psi(h, t)$, then the following conditions hold:

(1) $\psi($ id, $t)=$ id for each $t \in I$.

(2) $h_{0}=$ id for each $h \in \mathcal{P}$.

(3) $h_{1}=h$ on $B$ for each $h \in \mathcal{P}$.

(4) $h_{t}=$ id on $X \backslash B^{\prime}$ for all $h \in \mathcal{P}, t \in I$.

(5) As 3.2(5).

(6) As 3.2(6) but with $h_{t}=h_{t}^{\prime}$ on $Y$ for each $t \in I$.

(7) As 3.2(7), but we now define $f^{*}=\psi^{*}(f) \in I_{\Lambda \times I}(X)$ and consider Lipschitz properties of $f^{*} \mid Y \times \Lambda \times I \in I_{\Lambda \times I}(Y)$.

(8) As 3.2(8), but we now consider quasisymmetry properties of $h_{t} \mid Y \in$ $H(Y), t \in I$.

We let $\mathcal{D}_{Q S}^{*}(X ; \mathcal{Y})$ denote $\mathcal{D}^{*}(X ; \mathcal{Y})$ with $(7)$ deleted. 
3.7. Remarks. 1. The conditions (7a), (7b), (7c), (8a), (8b), and (8c) of Definitions 3.2, 3.4, and 3.6 (and the respective conditions of the results in later sections) will be considered independently of each other in the proofs. The weak local finiteness of $\mathcal{Y}$ will only be needed for (8c) in this section; otherwise the different elements of $\mathcal{Y}$ will be considered independently of each other.

2. In Section 5 we establish $\mathcal{D}\left(\mathbf{R}^{n} ; \mathcal{Y}_{n}\right)$ and $\mathcal{D}\left(\mathbf{R}_{+}^{n} ; \mathcal{Y}_{n}^{+}\right)$assuming, however, that $\operatorname{dim} Y \geq 2$ in $3.2(8 \mathrm{~b})$. Siebenmann and Sullivan [SS, Appendix B] proved essentially $\mathcal{D}(X ; \mathcal{Y})$, with (8) deleted and having in (7) only the LIP part (a), for pairs $(X, \mathcal{Y})$ described in 3.1 .

3 . Without altering any proof of this paper, we could strengthen the conditions (7b) and (7c) of Definitions 3.2, 3.4, and 3.6 (and the respective conditions of the results in later sections) to assert that $L^{*}$ can be chosen to be of the form $L^{*}=c L^{\nu}$ with $c, \nu \geq 1$ independent of $(\Lambda, f, L)$ (and depending only on $n$ in Section 6). Cf. 5.9.

3.8. Lemma. Let $\tau=\left(X ; A, A^{\prime}, U, B, B^{\prime} ; \mathcal{Y}\right)$ be as in 3.2 , let $U_{1}$ be an open neighbourhood of $B^{\prime}$ with compact closure in $U$, and construct $\tau_{1}$ from $\tau$ by replacing $U$ by $U_{1}$. Then $\mathcal{D}(\tau)$ follows from $\mathcal{D}_{1}\left(\tau_{1}\right)$.

Proof. Let $\mathcal{P}_{1}$ be the neighbourhood and $\varphi_{1}$ the map given by $\mathcal{D}_{1}\left(\tau_{1}\right)$. Then there is a neighbourhood $\mathcal{P}$ of id in $E\left(U, A^{\prime} ; X\right)$ such that $h \mid U_{1} \in \mathcal{P}_{1}$ for each $h \in \mathcal{P}$. Define a continuous map $\varphi: \mathcal{P} \times I \rightarrow E(U, A ; X)$ by

$$
\varphi(h, t)= \begin{cases}\varphi_{1}\left(h \mid U_{1}, t\right) & \text { on } U_{1}, \\ h & \text { on } U \backslash B^{\prime} .\end{cases}
$$

Then $\varphi$ trivially satisfies the conditions (1)-(8) of 3.2 with (7c) and (8c) deleted. If $\mathcal{P}$ is small enough, (7c) and (8c) also hold by 2.7 or, as $\left\{U_{1} \cap Y \mid Y \in \mathcal{Y}\right\}$ is finite, by 2.9 , respectively. $\square$

3.9. Lemma. The statements $\mathcal{D}(X ; \mathcal{Y})$ and $\mathcal{D}^{*}(X ; \mathcal{Y})$ are equivalent.

Proof. Let $\tau=\left(X ; A, A^{\prime}, U, B, B^{\prime} ; \mathcal{Y}\right)$ be as in 3.2 , and construct $\tau_{1}$ from $\tau$ by replacing $B$ by a compact neighbourhood $B_{1} \subset \subset B^{\prime}$ of $B$. We first show that $\mathcal{D}\left(\tau_{1}\right)$ implies $\mathcal{D}^{*}(\tau)$. Let $\mathcal{P}$ be the neighbourhood and $\varphi$ the map given by $\mathcal{D}\left(\tau_{1}\right)$. Choose $\mathcal{P}$ so small that $h B \subset B_{1}$ for each $h \in \mathcal{P}$. We define a function $\psi: \mathcal{P} \times I \rightarrow H(X, A)$ setting

$$
\psi(h, t)= \begin{cases}\varphi(h, t)^{-1} h & \text { on } U, \\ \mathrm{id} & \text { on } X \backslash B^{\prime} .\end{cases}
$$

Then $\psi$ is continuous by 2.11 . The conditions (1)-(8) of 3.6 with (7c) and (8c) deleted are easy to verify. If $\mathcal{P}$ is small enough, $(7 \mathrm{c})$ and $(8 \mathrm{c})$ also hold by 2.7 or 2.9 , respectively (to apply 2.9 , choose an open neighbourhood $V$ of $B^{\prime}$ with $\bar{V} \subset U$ compact). 
We now show that $\mathcal{D}^{*}\left(\tau_{1}\right)$ implies $\mathcal{D}(\tau)$. Let $\mathcal{P}$ be the neighbourhood and $\psi$ the map given by $\mathcal{D}^{*}\left(\tau_{1}\right)$. Choose $\mathcal{P}$ so small that $h B_{1} \supset B$ for each $h \in \mathcal{P}$. Then $\varphi: \mathcal{P} \times I \rightarrow E(U, A ; X)$, defined by $\varphi(h, t)=h \psi(h, t)^{-1} \mid U$, is the desired map. $\square$

3.10. Composition. Let $U \subset X$ be open and $B^{\prime} \subset U$ be compact. Consider $\mathcal{D}=\mathcal{D}\left(X ; \emptyset, \emptyset, U, \emptyset, B^{\prime} ; \mathcal{Y}\right)$. For $i=1,2$, let $\mathcal{P}_{i}$ be a subset of $E(U ; X)$ containing id and let $\varphi_{i}: \mathcal{P}_{i} \times I \rightarrow E(U ; X)$ be a continuous map satisfying $\mathcal{D}$. Suppose that $\varphi_{1}\left[\mathcal{P}_{1} \times 1\right] \subset \mathcal{P}_{2}$. Then we can define a continuous map $\varphi_{2} * \varphi_{1}: \mathcal{P}_{1} \times I \rightarrow E(U ; X)$ by

$$
\left(\varphi_{2} * \varphi_{1}\right)(h, t)= \begin{cases}\varphi_{1}(h, 2 t) & \text { if } 0 \leq t \leq \frac{1}{2} \\ \varphi_{2}\left(\varphi_{1}(h, 1), 2 t-1\right) & \text { if } \frac{1}{2} \leq t \leq 1\end{cases}
$$

and $\varphi_{2} * \varphi_{1}$ also satisfies $\mathcal{D}$, as 3.2(7) follows from 2.10.

If $Z \subset X$ is open, we let $\mathcal{Y} \mid Z$ denote the family $(Y \cap Z)_{Y \in \mathcal{Y}}$.

3.11. Lemma. Let $\mathcal{Z}=\{Z \mid Z \subset X$ open, $\mathcal{D}(Z ; \mathcal{Y} \mid Z)$ holds $\}$.

(i) If $X_{1} \in \mathcal{Z}$ and $X_{0} \subset X_{1}$ is open, then $X_{0} \in \mathcal{Z}$.

(ii) If $X_{1}, X_{2} \in \mathcal{Z}$, then $X_{1} \cup X_{2} \in \mathcal{Z}$.

Proof. Since $\left(\mathcal{Y} \mid Z_{1}\right)\left|Z_{0}=\mathcal{Y}\right| Z_{0}$ for open subsets $Z_{0} \subset Z_{1}$ of $X$, we may assume that $X_{1}=X$ in (i) and $X_{1} \cup X_{2}=X$ in (ii).

(i): To establish $\mathcal{D}\left(X_{0} ; \mathcal{Y} \mid X_{0}\right)$, we prove $\mathcal{D}\left(X_{0} ; A, A^{\prime}, U, B, B^{\prime} ; \mathcal{Y} \mid X_{0}\right)$. Note that $E\left(U ; X_{0}\right)$ can be considered as a subspace of $E(U ; X)$. We can apply $\mathcal{D}\left(X ; A \cap B^{\prime}, \bar{A}^{\prime}, U, B, B^{\prime} ; \mathcal{Y}\right)$; let $\mathcal{P}$ be the neighbourhood and $\varphi$ the map given by this statement. Then $\mathcal{P}_{0}=\left\{h \in \mathcal{P} \mid h U \subset X_{0}\right\}$ is a neighbourhood of id: $U \rightarrow X_{0}$ in $E\left(U, A^{\prime} ; X_{0}\right)$. Through restriction, $\varphi$ defines a continuous map $\varphi_{0}: \mathcal{P}_{0} \times I \rightarrow E\left(U, A ; X_{0}\right)$, which is the desired one.

(ii): To prove $\mathcal{D}(X ; \mathcal{Y})$, we prove $\mathcal{D}\left(X ; A, A^{\prime}, U, B, B^{\prime} ; \mathcal{Y}\right)$. It follows from 3.8 and 3.5.2 that we may assume $\bar{U}$ to be compact and that it suffices to construct the desired neighbourhood $\mathcal{P}$ of id in $E\left(U, A^{\prime} ; X\right)$ to be a uniform neighbourhood only.

Choose a closed neighbourhood $A^{\prime \prime} \subset \subset A^{\prime}$ of $A$ in $X$. Choose open covers $\left\{X_{1}^{\prime}, X_{2}^{\prime}\right\}$ and $\left\{X_{1}^{\prime \prime}, X_{2}^{\prime \prime}\right\}$ of $X$ with $X_{i}^{\prime \prime} \subset \subset X_{i}^{\prime} \subset \subset X_{i}$ for $i=1,2$. Let $U_{i}=$ $U \cap X_{i}^{\prime}$; then $U \equiv U_{1} \cup U_{2}$, and $\bar{U}_{i}$ is a compact set in $X_{i}$, whence $d\left(U_{i}, X \backslash X_{i}\right)>0$. Let $B_{i}=B \cap \bar{X}_{i}^{\prime \prime}$; then $B=B_{1} \cup B_{2}$, and $B_{i}$ is a compact set in $U_{i}$. Choose compact sets $B_{1}^{\prime}, B_{1}^{*}$, and $B_{2}^{\prime}$ with $B_{1} \subset \subset B_{1}^{*} \subset \subset B_{1}^{\prime} \subset U_{1} \cap B^{\prime}$ and with $B_{2} \subset \subset B_{2}^{\prime} \subset U_{2} \cap B^{\prime}$.

We first apply $\mathcal{D}\left(X_{1} ; A^{\prime \prime} \cap X_{1}, A^{\prime} \cap X_{1}, U_{1}, B_{1}^{*}, B_{1}^{\prime} ; \mathcal{Y} \mid X_{1}\right)$; let $\mathcal{P}_{1}$ be the neighbourhood and $\varphi_{1}$ the map thus obtained. Choose a uniform neighbourhood $\mathcal{P}$ of id in $E\left(U, A^{\prime} ; X\right)$ such that $h U_{1} \subset X_{1}$ and $h \mid U_{1} \in \mathcal{P}_{1}$ for each $h \in \mathcal{P}$. Define a continuous map $\varphi_{1}^{\prime}: \mathcal{P} \times I \rightarrow E\left(U, A^{\prime \prime} ; X\right)$ letting $\varphi_{1}^{\prime}(h, t)$ be $\varphi_{1}\left(h \mid U_{1}, t\right)$ on $U_{1}$ and $h$ on $U \backslash B_{1}^{\prime}$. Choosing $\mathcal{P}$ small enough, $\varphi_{1}^{\prime}$ satisfies $\mathcal{D}\left(X ; A^{\prime \prime}, A^{\prime}, U, B_{1}^{*}, B_{1}^{\prime} ; \mathcal{Y}\right) ;$ cf. the proof of 3.8 . 
We now apply $\mathcal{D}\left(X_{2} ;\left(A \cup B_{1}\right) \cap X_{2},\left(A^{\prime \prime} \cup B_{1}^{*}\right) \cap X_{2}, U_{2}, B_{2}, B_{2}^{\prime} ; \mathcal{Y} \mid X_{2}\right)$; let $\mathcal{P}_{2}$ be the neighbourhood and $\varphi_{2}$ the map thus obtained. Choose a uniform neighbourhood $\mathcal{Q}$ of id in $E\left(U, A^{\prime \prime} \cup B_{1}^{*} ; X\right)$ such that $h U_{2} \subset X_{2}$ and $h \mid U_{2} \in \mathcal{P}_{2}$ for each $h \in \mathcal{Q}$. Define a continuous map $\varphi_{2}^{\prime}: \mathcal{Q} \times I \rightarrow E\left(U, A \cup B_{1} ; X\right)$ letting $\varphi_{2}^{\prime}(h, t)$ be $\varphi_{2}\left(h \mid U_{2}, t\right)$ on $U_{2}$ and $h$ on $U \backslash B_{2}^{\prime}$. Choosing $\mathcal{Q}$ small enough, $\varphi_{2}^{\prime}$ satisfies $\mathcal{D}\left(X ; A \cup B_{1}, A^{\prime \prime} \cup B_{1}^{*}, U, B_{2}, B_{2}^{\prime} ; \mathcal{Y}\right)$; cf. the proof of 3.8 .

Choosing $\mathcal{P}$ small enough, we have that $\varphi_{1}^{\prime}[\mathcal{P} \times 1] \subset \mathcal{Q}$. Then $\varphi=\varphi_{2}^{\prime} * \varphi_{1}^{\prime}$ is the desired map. $\square$

3.12. Lemma. Suppose that the family $\mathcal{Z}$ of 3.11 covers $X$. Then $X \in \mathcal{Z}$.

Proof. First note that by 3.11 every open subset of $X$ with compact closure belongs to $\mathcal{Z}$. To prove $\mathcal{D}(X ; \mathcal{Y})$, we establish $\mathcal{D}\left(X ; A, A^{\prime}, U, B, B^{\prime} ; \mathcal{Y}\right)$. By 3.8 and 3.5.2, we may assume that $\bar{U}$ is compact, in which case there is an open neighbourhood $X_{0}$ of $\bar{U}$ with $\bar{X}_{0}$ compact, and then, in addition, it suffices to construct the desired neighbourhood $\mathcal{P}$ of id: $U \rightarrow X$ to be only a neighbourhood of id in the subspace $\mathcal{P}_{0}$ of $E\left(U, A^{\prime} ; X\right)$ consisting of elements mapping $U$ into $X_{0}$. Since $\mathcal{P}_{0}=E\left(U, A^{\prime} \cap X_{0} ; X_{0}\right)$ and since $E\left(U, A \cap X_{0} ; X_{0}\right)$ is a subspace of $E(U, A ; X)$, the neighbourhood $\mathcal{P}$ and the map $\varphi$ given by the statement $\mathcal{D}\left(X_{0} ; A \cap X_{0}, A^{\prime} \cap X_{0}, U, B, B^{\prime} ; \mathcal{Y} \mid X_{0}\right)$, valid as $X_{0} \in \mathcal{Z}$, are what we sought. $\square$

3.13. Lemma. The statement $\mathcal{D}(X ; \mathcal{Y})$ is a LIP invariant of the pair $(X, \mathcal{Y})$. More exactly, if $\mathcal{D}(X ; \mathcal{Y})$ holds, $X^{\prime}$ is a metric space, $g: X \rightarrow X^{\prime}$ is a LIP homeomorphism, and $\mathcal{Y}^{\prime}=\{g Y \mid Y \in \mathcal{Y}\}$, then $\mathcal{D}\left(X^{\prime} ; \mathcal{Y}^{\prime}\right)$ holds. The statement $\mathcal{D}_{Q S}(X ; \mathcal{Y})$ is an LQS invariant of $(X, \mathcal{Y})$.

Proof. Obviously, $\mathcal{D}(X ; \mathcal{Y})$ is a BL invariant of $(X, \mathcal{Y})$. The LIP invariance then follows from 3.11(i) and 3.12. The second part is proved similarly; recall only 3.7.1. व

\section{Handle lemmas}

4.1. In this section we prove the inductive handle lemmas 4.2 and 4.3. For integers $0 \leq p \leq n \geq 1$, we set $\mathcal{Y}_{n p}=\left\{Y \in \mathcal{Y}_{n} \mid \mathbf{R}^{p} \subset Y\right\}$ and $\mathcal{Y}_{n p}^{+}=\left\{Y \in \mathcal{Y}_{n}^{+} \mid\right.$ $\left.\mathbf{R}^{p} \subset Y\right\}$, where the families $\mathcal{Y}_{n}$ and $\mathcal{Y}_{n}^{+}$are defined in 2.1.

Convention. From now on in this paper, we will tacitly assume that in the condition (8b) of Definitions 3.2, 3.4, and 3.6, the set $Y$, which will always be a manifold, is of dimension at least two.

Under this convention, the lemmas in Section 3 are still valid by 3.7.1.

4.2. Lemma. Let $0 \leq m \leq p \leq n \geq 1$ be integers. Let $X=\mathbf{R}^{n}=$ $\mathbf{R}^{m} \times \mathbf{R}^{p-m} \times \mathbf{R}^{n-p}$ and $\mathcal{Y}=\mathcal{Y}_{n p}$. Suppose that $\mathcal{D}\left(\mathbf{R}^{n} \backslash \mathbf{R}^{p} ; \mathcal{Y} \mid \mathbf{R}^{n} \backslash \mathbf{R}^{p}\right)$ holds. Then $\mathcal{D}\left(X ; A, A^{\prime}, U, B, B^{\prime} ; \mathcal{Y}\right)$ holds, where

$$
A=\mathbf{R}^{m} \times\left(\mathbf{R}^{p-m} \backslash B^{p-m}\right) \times \mathbf{R}^{n-p},
$$




$$
\begin{aligned}
A^{\prime} & =\mathbf{R}^{m} \times\left(\mathbf{R}^{p-m} \backslash B^{p-m}\left(\frac{1}{2}\right)\right) \times \mathbf{R}^{n-p} \\
U & =5 B^{m} \times 5 B^{p-m} \times 5 B^{n-p} \\
B & =\bar{B}^{m}\left(\frac{1}{2}\right) \times \bar{B}^{p-m} \times \bar{B}^{n-p}\left(\frac{1}{2}\right) \\
B^{\prime} & =\bar{B}^{m} \times 2 \bar{B}^{p-m} \times 2 \bar{B}^{n-p}
\end{aligned}
$$

4.3. Lemma. Let $0 \leq m \leq p<n$ be integers. Let $X=\mathbf{R}_{+}^{n}=\mathbf{R}^{m} \times \mathbf{R}^{p-m} \times$ $\mathbf{R}_{+}^{n-p}$ and $\mathcal{Y}=\mathcal{Y}_{n p}^{+}$. Suppose that $\mathcal{D}\left(\mathbf{R}_{+}^{n} \backslash \mathbf{R}^{p} ; \mathcal{Y} \mid \mathbf{R}_{+}^{n} \backslash \mathbf{R}^{p}\right)$ holds. Then $\mathcal{D}(X ; A$, $\left.A^{\prime}, U, B, B^{\prime} ; \mathcal{Y}\right)$ holds, where the sets $A, A^{\prime}, U, B, B^{\prime}$ are the intersections of the respective sets in 4.2 with $\mathbf{R}_{+}^{n}$. 4.3 .

4.4. Remarks. 1 . We only prove 4.2 ; obvious modifications give a proof for

2. As the construction will show, the following condition $\left(8^{\prime}\right)$ can be added to the list of 3.2 for the claimed deformation statements in 4.2 and 4.3 (the case $p=0)$ :

$\left(8^{\prime}\right)$ Let $Y_{1}, Y_{2} \in \mathcal{Y}$ be distinct and homeomorphic to $\mathbf{R}_{+}^{1}$, and let $Y=$ $Y_{1} \cup Y_{2}$. If $h \in \mathcal{P}$ respects $Y_{1}$ and $Y_{2}$, if $h \mid U \cap Y$ is (a) LQS or (c) $\eta-Q S$, and if $t \in I$, then $h_{t} \mid U \cap Y \in E(U \cap Y ; Y)$ is, respectively, (a) LQS or (c) $\eta_{*}-\mathrm{QS}$ with $\eta_{*}$ not depending on $(h, t)$.

4.5. Hyperbolic geometry. We recall some constructions from $\left[\mathrm{TV}_{2}, 2.3-2.11\right]$.

For $m \geq 1$, we let $\mathrm{Möb}_{m}$ denote the subgroup of $H\left(\bar{B}^{m}\right)$ of all Möbius transformations of $\bar{B}^{m}$.

For $n \geq m \geq 1$, we let $\sigma$ denote the restriction to $\bar{B}^{n} \backslash S^{m-1}$ of the hyperbolic metric of $\mathbf{R}^{n} \cup\{\infty\} \backslash S^{m-1}$ defined in $\left[\mathrm{TV}_{2}, 2.4\right]$. The hyperbolic metric $\sigma$ is invariant under each $g \in \mathrm{Möb}_{n}$ that respects $S^{m-1}$, and $\sigma$ is LIP equivalent to the Euclidean metric; in particular, $|x-y| \leq \sigma(x, y)$ for $x, y \in \bar{B}^{n} \backslash S^{m-1}$, as follows from $\left[\mathrm{TV}_{2},(2.6)\right]$. The case $m=n$ gives the hyperbolic metric on $B^{m}$; now every hyperbolic ball $B_{\sigma}(x, r)$ is a Euclidean ball.

Let $m \geq 1$. Let $G$ be a discrete subgroup of $\mathrm{Möb}_{m}$ acting freely on $B^{m}$. Let $Q$ be the orbit space $B^{m} / G$ with the quotient topology. Then the natural map $\pi_{0}: B^{m} \rightarrow Q$ is a covering map, and $Q$ is an $m$-manifold without boundary. Moreover, $\pi_{0}$ defines a $C^{\infty}$ - (and hence a LIP) structure on $Q$. The hyperbolic metric $\sigma_{Q}$ on $Q$ is defined as follows: If $q, q^{\prime} \in Q, \sigma_{Q}\left(q, q^{\prime}\right)$ is the hyperbolic distance of $\pi_{0}^{-1}(q)$ and $\pi_{0}^{-1}\left(q^{\prime}\right)$ as subsets of $B^{m}$. Then $\pi_{0}$ is locally a hyperbolic isometry. Thus the LIP structure on $Q$ is also defined by the metric $\sigma_{Q}$. As in $\left[\mathrm{TV}_{2}, 2.9\right]$, we say that $G$ is a Sullivan group and $Q$ a Sullivan manifold if $Q$ is compact and if for some (and hence for every) point $q \in Q$ there is a LIP

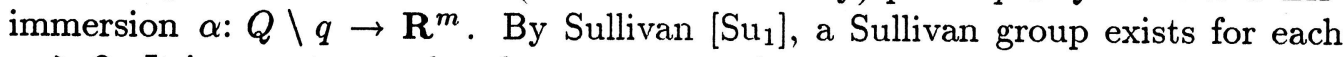
$m \geq 2$. It is easy to see that for $m=1$, a subgroup of Möb 1 is a Sullivan group if and only if it is generated by one nonidentical increasing map. Every Sullivan 1-manifold is $C^{\infty}$-diffeomorphic to $S^{1}$. We fix for each $m \geq 1$ a Sullivan group 
$G^{m}$ and the corresponding Sullivan manifold $Q^{m}=B^{m} / G^{m}$. Then for each $m$, by the compactness of $Q^{m}$ there is $r_{m} \in\left(0, \frac{1}{2}\right)$ such that $\pi_{0}: B^{m} \rightarrow Q^{m}$ is a hyperbolic isometry on a neighbourhood of each closed hyperbolic ball $\bar{B}_{\sigma}\left(x, r_{m}\right)$, $x \in B^{m}$.

Let $0 \leq m \leq n \geq 1$. Set $k=n-m$ and $A_{k}^{n}=\bar{B}^{n} \backslash S^{m-1}$ (with $S^{-1}=\emptyset$ ). Then there is a natural homeomorphism $u: A_{k}^{n} \rightarrow B^{m} \times \bar{B}^{k} \subset \mathbf{R}^{n}$ defined by the so-called Möbius coordinates of $A_{k}^{n}$ whenever $m, k>0\left[\mathrm{TV}_{2}, 2.7\right.$ and 2.10] or by $u=$ id whenever $m=0$ or $k=0$. Clearly $u$ respects each $Y \in \mathcal{Y}_{n}$. For $m \geq 1$, recall the hyperbolic metrics $\sigma$ of $A_{k}^{n}$ and $\sigma$ of $B^{m}$; for $m=0$, let $\sigma$ denote the Euclidean metric. Define a metric $\varrho_{0}$ on $B^{m} \times \bar{B}^{k}$ by

$$
\varrho_{0}\left(x, x^{\prime}\right)=\max \left(\sigma\left(x_{1}, x_{1}^{\prime}\right),\left|x_{2}-x_{2}^{\prime}\right|\right) \text {. }
$$

Then $u:\left(A_{k}^{n}, \sigma\right) \rightarrow\left(B^{m} \times \bar{B}^{k}, \varrho_{0}\right)$ is BL by $\left[\mathrm{TV}_{2}, 2.11\right]$.

Now let $0 \leq m \leq p \leq n \geq 1$. Set $k=n-m$ as above. Let $\widetilde{B}^{k}=\bar{B}^{p-m} \times$ $\bar{B}^{n-p}$. We introduce the norm $\|(x, y)\|=\max (|x|,|y|)$ on $\mathbf{R}^{k}=\mathbf{R}^{p-m} \times \mathbf{R}^{n-p}$ and define a BL homeomorphism $\tau_{0}:\left(\mathbf{R}^{k},|\cdot|\right) \rightarrow\left(\mathbf{R}^{k},\|\cdot\|\right)$ setting $\tau_{0}(0)=0$ and $\tau_{0}(x)=|x| x /\|x\|$ for $x \neq 0$. Then $\tau_{0} \bar{B}^{k}=\widetilde{B}^{k}$. Define a metric $\varrho$ on $B^{m} \times \mathbf{R}^{k}$ by

$$
\varrho\left(x, x^{\prime}\right)=\max \left(\sigma\left(x_{1}, x_{1}^{\prime}\right),\left\|x_{2}-x_{2}^{\prime}\right\|\right) .
$$

Replacing here $\sigma$ by $\sigma_{Q}$ (if $m \geq 1$ ), we obtain a metric on $Q^{m} \times \mathbf{R}^{k}$, also denoted by $\varrho$, with which $Q^{m} \times \mathbf{R}^{k}$ will be considered. Let $B^{*}=B^{m} \times \widetilde{B}^{k}$; then the homeomorphism $\tau=\mathrm{id} \times \tau_{0}:\left(B^{m} \times \bar{B}^{k}, \varrho_{0}\right) \rightarrow\left(B^{*}, \varrho\right)$ is BL and respects each $Y \in \mathcal{Y}_{n}$. We obtain a $c_{n}$-BL homeomorphism $v=\tau u:\left(A_{k}^{n}, \sigma\right) \rightarrow\left(B^{*}, \varrho\right)$, where $c_{n}$ depends only on $n$. Suppose $m \geq 1$. Let $Q^{*}=Q^{m} \times \widetilde{B}^{k}$. We define covering maps $\pi=\pi_{0} \times$ id: $B^{*} \rightarrow Q^{*}$ and $e=\pi v: A_{k}^{n} \rightarrow Q^{*}$. For each $Y=\mathbf{R}^{m} \times Y_{1} \in \mathcal{Y}_{n m}$, where $Y_{1} \subset \mathbf{R}^{k}$, we define a subset $Y^{*}=Q^{m} \times Y_{1}$ of $Q^{m} \times \mathbf{R}^{k}$. Then $e^{-1}\left[Q^{*} \cap Y^{*}\right]=v^{-1}\left[B^{*} \cap Y\right]=A_{k}^{n} \cap Y$ for each $Y \in \mathcal{Y}_{n m}$.

4.6. Lifting homeomorphisms. Let $1 \leq m \leq p \leq n$ and

$$
\mathcal{P}_{0}=\left\{h \in H\left(Q^{*}, \partial Q^{*}\right) \mid \varrho(h, \mathrm{id})<\frac{1}{2} r_{m}\right\} .
$$

Note that $\mathcal{P}_{0}^{-1}=\mathcal{P}_{0}$. Each $h \in \mathcal{P}_{0}$ has a unique lift to a continuous map $\hat{h}: B^{*} \rightarrow B^{*}$ such that $\pi \hat{h}=h \pi$ and such that $\hat{h}(0) \in B_{\sigma}\left(0, \frac{1}{2} r_{m}\right) \times \widetilde{B}^{k}$. In fact, if $x \in B^{m}$ and if

$$
\pi_{x}:\left(B_{\sigma}\left(x, r_{m}\right) \times \widetilde{B}^{k}, \varrho\right) \rightarrow\left(B_{\sigma_{Q}}\left(\pi_{0}(x), r_{m}\right) \times \widetilde{B}^{k}, \varrho\right)
$$

is the isometric homeomorphism defined by $\pi$, then $\hat{h}=\pi_{x}^{-1} h \pi_{x}$ on $\bar{B}_{\sigma}\left(x, \frac{1}{4} r_{m}\right) \times$ $\widetilde{B}^{k}$. This description implies that $\varrho\left(\hat{h}_{1}, \hat{h}_{2}\right)=\varrho\left(h_{1}, h_{2}\right)$ for all $h_{1}, h_{2} \in \mathcal{P}_{0}$. In 
particular, $\varrho(\hat{h}$, id $)<\frac{1}{2} r_{m}$ for each $h \in \mathcal{P}_{0}$. It is easy to see that if $h \in \mathcal{P}_{0}$, then $\widehat{h^{-1}}=\hat{h}^{-1}$, whence $\hat{h} \in H\left(B^{*}, \partial B^{*}\right)$.

For $h \in \mathcal{P}_{0}$ we set $h^{*}=v^{-1} \hat{h} v \in H\left(A_{k}^{n}, \partial A_{k}^{n}\right)$. Then $h^{*}$ is an $e$-lift of $h$, i.e., $e h^{*}=h e$. There is a constant $M_{n}$ depending only on $n$ such that $\sigma\left(h^{*}\right.$,id $) \leq M_{n}$ for all $h \in \mathcal{P}_{0}$. Thus, every $h^{*}$ can be extended by the identity to a homeomorphism $\bar{h}: \mathbf{R}^{n} \rightarrow \mathbf{R}^{n}$. Note that $\bar{h}^{-1}=\overline{h^{-1}}$ for $h \in \mathcal{P}_{0}$.

4.7. Lemma. The relation $h \mapsto \bar{h}$ is a continuous map $\mathcal{P}_{0} \rightarrow H\left(\mathbf{R}^{n}, \mathbf{R}^{n} \backslash B^{n}\right)$ sending id to id and satisfying the following conditions:

(1) Let $Y \in \mathcal{Y}_{n m}$. If $h \in \mathcal{P}_{0}$ respects $Q^{*} \cap Y^{*}$, then $\bar{h}$ respects $Y$. If $h_{1}, h_{2} \in \mathcal{P}_{0}$ respect $Q^{*} \cap Y^{*}$ and $h_{1}=h_{2}$ on $Q^{*} \cap Y^{*}$, then $\bar{h}_{1}=\bar{h}_{2}$ on $Y$.

(2) Let $\Lambda$ be a metric space, and let $f=\left(f_{\lambda}\right)_{\lambda \in \Lambda} \in I_{\Lambda}\left(Q^{*}\right)$ with $f_{\lambda} \in \mathcal{P}_{0}$ for each $\lambda \in \Lambda$. Define $\bar{f}=\left(\bar{f}_{\lambda}\right)_{\lambda \in \Lambda} \in I_{\Lambda}\left(\mathbf{R}^{n}\right)$. Suppose that $Y \in \mathcal{Y}_{n m}$, that $f$ respects $\left(Q^{*} \cap Y^{*}\right) \times \Lambda$, and that $f \mid\left(Q^{*} \cap Y^{*}\right) \times \Lambda$ is (a) LIP or (b) locally $L$ BL or (c) $L-\mathrm{BL}$. Then there is an open cover $\left(\Lambda_{j}\right)$ of $\Lambda$, consisting only of $\Lambda$ in the case (c), such that $\bar{f} \mid Y \times \Lambda_{j}$ is for each $j$, respectively, (a) BL or (b), (c) $\bar{L}$ BL with $\bar{L}$ depending only on $L$ and $n$.

(3) If $Y \in \mathcal{Y}_{n m}, l=\operatorname{dim} Y, h \in \mathcal{P}_{0}, h$ respects $Q^{*} \cap Y^{*}$, and $h \mid Q^{*} \cap Y^{*}$ is (a) LQS or (b) locally $\eta$-QS or (c) $\eta-Q S$, then $\bar{h} \mid Y$ is, respectively, (a) QS or (b) (if $l \geq 2$ ), (c) $\bar{\eta}$-QS with $\bar{\eta}$ depending only on $\eta$ and $n$. Here it is provided that if $l=1$, then $\varrho(h$,id $)<r_{0}$, where $r_{0}>0$ is an absolute constant.

Proof. The continuity of $h \mapsto \bar{h}$ follows from the fact that $d\left(\bar{h}, \bar{h}^{\prime}\right) \leq c_{n} \varrho\left(h, h^{\prime}\right)$ for $h, h^{\prime} \in \mathcal{P}_{0}$. Obviously, $\overline{\mathrm{id}}=\mathrm{id}$ is true and (1) holds. Consider $Y \in \mathcal{Y}_{n m}$. Then $e$ defines a $c_{n}-\mathrm{BL}$ homeomorphism

$$
e_{x}:\left(u^{-1}\left[B_{\sigma}\left(x, r_{m}\right) \times \bar{B}^{k}\right] \cap Y, \sigma\right) \rightarrow\left(\left(B_{\sigma_{Q}}\left(\pi_{0}(x), r_{m}\right) \times \widetilde{B}^{k}\right) \cap Y^{*}, \varrho\right)
$$

for all $x \in B^{m}$. Consider $h \in \mathcal{P}_{0}$ that respects $Q^{*} \cap Y^{*}$. Then $\bar{h}$ defines a homeomorphism $h_{Y} \in H\left(A_{k}^{n} \cap Y\right)$ with

$$
h_{Y}=e_{x}^{-1}\left(h \mid Q^{*} \cap Y^{*}\right) e_{x} \quad \text { on } u^{-1}\left[\bar{B}_{\sigma}\left(x, \frac{1}{4} r_{m}\right) \times \bar{B}^{k}\right] \cap Y
$$

if $x \in B^{m}$.

(2b) for $\Lambda$ a singleton: Let $Y$ and $h$ be as just with $h \mid Q^{*} \cap Y^{*}$ being locally $L$-BL. Then $h_{Y}$ is locally $L^{\prime}-\mathrm{BL}, L^{\prime}=c_{n}^{2} L$, in the hyperbolic metric $\sigma$ of $A_{k}^{n}$. Since $A_{k}^{n} \cap Y$ is a convex subset of $\mathbf{R}^{n}$, the proof of $\left[\mathrm{TV}_{2}, 2.12\right]$ shows that $h_{Y}$ is $\bar{L}-\mathrm{BL}, \bar{L}=2 L^{\prime} e^{M_{n}}$, in the Euclidean metric. Hence, $\bar{h} \mid Y$ is $\bar{L}-\mathrm{BL}$.

(2): In the cases (a) and (b), the compactness of $Q^{*} \cap Y^{*}$ implies that for each $\lambda \in \Lambda$ there are a neighbourhood $\Lambda_{\lambda}$ of $\lambda$, a finite open cover $\mathcal{V}_{\lambda}$ of $Q^{*} \cap Y^{*}$, and a number $L_{\lambda} \geq 1$, equal to $L$ in (b), such that $f \mid V \times \Lambda_{\lambda}$ and $f^{-1} \mid V \times \Lambda_{\lambda}$ are $L_{\lambda}$-Lipschitz for each $V \in \mathcal{V}_{\lambda}$. Thus, in (2) we may assume that there is $L \geq 1$ such that $f_{\lambda} \mid Q^{*} \cap Y^{*}$ is locally $L-B L$ for each $\lambda \in \Lambda$ and such that $f \mid\{x\} \times \Lambda$ 
and $f^{-1} \mid\{x\} \times \Lambda$ are $L$-Lipschitz for each $x \in Q^{*} \cap Y^{*}$, and it suffices to prove that $\bar{f} \mid Y \times \Lambda$ is then $\widehat{L}-\mathrm{BL}$ with $\widehat{L}$ depending only on $L$ and $n$. By the special case of (b) above, $\bar{f}_{\lambda} \mid Y$ is $\bar{L}$-BL for each $\lambda \in \Lambda$. Obviously,

$$
d\left(\bar{f}_{\lambda}, \bar{f}_{\lambda^{\prime}} ; Y\right) \leq c_{n} \varrho\left(f_{\lambda}, f_{\lambda^{\prime}} ; Q^{*} \cap Y^{*}\right) \leq c_{n} L d\left(\lambda, \lambda^{\prime}\right)
$$

for all $\lambda, \lambda^{\prime} \in \Lambda$. It easily follows that $\bar{f} \mid Y \times \Lambda$ is $\widehat{L}$-Lipschitz with $\widehat{L}=\bar{L}+c_{n} L$. Thus, also $(\bar{f} \mid Y \times \Lambda)^{-1}=\overline{f^{-1}} \mid Y \times \Lambda$ is $\widehat{L}$-Lipschitz.

(3): As $Q^{*} \cap Y^{*}$ is compact, (a) reduces to (c). Suppose first $l \geq 2$. In (b) and (c), $h_{Y}$ is locally $\eta_{1}-\mathrm{QS}, \eta_{1}(t)=c_{n}^{2} \eta\left(c_{n}^{2} t\right)$ for $t \geq 0$, in the hyperbolic metric $\sigma$ of $A_{k}^{n}$. If $x \in A_{k}^{n}$, by $\left[\mathrm{TV}_{2},(2.6)\right]$ the quotient $\sigma(x, y) /|x-y|$ has a finite positive limit as $y \rightarrow x$ in $A_{k}^{n}$. This implies that $H\left(x, h_{Y}\right) \leq \eta_{1}(1)$ for each $x \in \operatorname{Int}\left(A_{k}^{n} \cap Y\right)$. Here $H\left(x, h_{Y}\right)$ denotes the linear dilatation (in the Euclidean metric) of $h_{Y}$ at $x$ in the sense of [Vä1, 22.2]. Thus, $h_{Y} \mid \operatorname{Int}\left(A_{k}^{n} \cap Y\right)$ is $K-\mathrm{QC}$, $K=\eta_{1}(1)^{l-1}$, by [V $\left.\ddot{a}_{1}, 34.2\right]$. Hence, $\bar{h} \mid Y \in H(Y)$ is $K-\mathrm{QC}$ by [V $\left.\ddot{a}_{1}, 35.1\right]$. By $2.3, \bar{h} \mid Y$ is $\bar{\eta}$-QS with $\bar{\eta}$ depending only on $K$ and $l$.

Suppose now $l=1$. Then $m=1, Y=\mathbf{R}^{1}, A_{k}^{n} \cap Y=B^{1}$, and $Q^{*} \cap Y^{*}=$ $Y^{*}=Q^{1}$, where $Q^{1}$ is isometrically identified with the subspace $Q^{1} \times 0$ of $Q^{*}$. Moreover, $v \mid B^{1}=\mathrm{id}$ and $\pi \mid B^{1}=\pi_{0}$. Thus, letting $h_{1}=h \mid Q^{1} \in H\left(Q^{1}\right)$, we have that $\bar{h} \mid B^{1} \in H\left(B^{1}\right)$ is the $\pi$-lift $\hat{h}_{1}$ of $h_{1}$ defined in 4.6 (the case $m=p=n=1$ ). Since $h_{1}$ is $\eta$-QS, it follows that $\hat{h}_{1} \mid \bar{B}_{\sigma_{1}}\left(x, \frac{1}{4} r_{1}\right)$ is $\eta$-QS in the hyperbolic metric $\sigma_{1}$ of $B^{1}$ for each $x \in B^{1}$. Furthermore, $\sigma_{1}\left(\hat{h}_{1}\right.$, id $)=\sigma_{Q}\left(h_{1}\right.$, id $) \leq \varrho(h$, id $)$. Hence, by $[\mathrm{Lu}, 3.2]$ there is an absolute constant $r_{0} \in\left(0, \frac{1}{2} r_{1}\right]$ such that if $\varrho(h$, id $)<r_{0}$, then $\bar{h} \mid \mathbf{R}^{1}$ is $\bar{\eta}-\mathrm{QS}$ with $\bar{\eta}$ depending only on $\eta(1)$. 口

4.8. From now on we assume the situation of 4.2. Recall $\mathcal{Y}=\mathcal{Y}_{n p}$, and let $\mathcal{Y}^{*}=\left(Y^{*}\right)_{Y \in \mathcal{Y}}$. holds.

4.9. Lemma. Let $m \geq 1$ and $\widehat{Q}=Q^{m} \times\left(\mathbf{R}^{n-m} \backslash \mathbf{R}^{p-m}\right)$. Then $\mathcal{D}^{*}\left(\widehat{Q} ; \mathcal{Y}^{*} \mid \widehat{Q}\right)$

Proof. Let $\widehat{R}=\mathbf{R}^{n} \backslash \mathbf{R}^{p}=\mathbf{R}^{m} \times\left(\mathbf{R}^{n-m} \backslash \mathbf{R}^{p-m}\right)$. Choose an open cover $\left\{Q_{1}\right.$, $\left.\ldots, Q_{s}\right\}$ of $Q^{m}$ such that for each $i \in\{1, \ldots, s\}$ there is a LIP homeomorphism $f_{i}: Q_{i} \rightarrow \mathbf{R}^{m}$. Let $\widehat{Q}_{i}=Q_{i} \times\left(\mathbf{R}^{n-m} \backslash \mathbf{R}^{p-m}\right)$. Then $\hat{f}_{i}=f_{i} \times$ id: $\widehat{Q}_{i} \rightarrow \widehat{R}$ is a LIP homeomorphism with $\hat{f}_{i}\left[\widehat{Q}_{i} \cap Y^{*}\right]=\widehat{R} \cap Y$ for each $Y \in \mathcal{Y}$. Thus, as $\mathcal{D}(\widehat{R} ; \mathcal{Y} \mid \widehat{R})$ holds by the assumption of $4.2, \mathcal{D}\left(\widehat{Q}_{i} ; \mathcal{Y}^{*} \mid \widehat{Q}_{i}\right)$ holds by 3.13 . As $\left\{\widehat{Q}_{1}, \ldots, \widehat{Q}_{s}\right\}$ is an open cover of $\widehat{Q}$, the lemma follows by 3.11 (ii) and 3.9.

4.10. A modification. Let $m \geq 1$. We let $D^{m}$ denote the hyperbolic ball $B_{\sigma}\left(0, r_{m}\right) \subset B^{m}$. Choose a radial BL homeomorphism $\beta_{0}: \mathbf{R}^{m} \rightarrow \mathbf{R}^{m}$ such that $\beta_{0} \mid \mathbf{R}^{m} \backslash B^{m}=$ id and $\beta_{0} \bar{B}^{m}\left(\frac{1}{2}\right)=\bar{D}^{m}$. Then $\beta=\beta_{0} \times$ id: $\mathbf{R}^{n}=\mathbf{R}^{m} \times \mathbf{R}^{n-m} \rightarrow$ $\mathbf{R}^{n}$ is $\mathrm{BL}$, and $\beta Y=Y$ for each $Y \in \mathcal{Y}_{n}$. Thus, by the aid of $\beta$ we can replace $\bar{B}^{m}\left(\frac{1}{2}\right)$ by $\bar{D}^{m}$ in the definition of $B$ in 4.2 .

The next lemma is the core of the proof of 4.2. 
4.11. Lemma. There are a neighbourhood $\mathcal{P}$ of id in $E\left(U, A^{\prime} ; X\right)$ and a continuous map $\varphi_{0}: \mathcal{P} \rightarrow H\left(\mathbf{R}^{n}, \mathbf{R}^{n} \backslash B^{n}\right)$ with $\varphi_{0}(\mathrm{id})=\mathrm{id}$ which satisfy the following conditions:

(1) $\varphi_{0}(h)=v^{-1} h v$ on $v^{-1} B$ for each $h \in \mathcal{P}$.

(2) If $h \in \mathcal{P}$ respects $Y \in \mathcal{Y}$, so does $\varphi_{0}(h)$. If $h, h^{\prime} \in \mathcal{P}$ respect $Y \in \mathcal{Y}$ and $h=h^{\prime}$ on $U \cap Y$, then $\varphi_{0}(h)=\varphi_{0}\left(h^{\prime}\right)$ on $Y$.

(3) Let $\Lambda, f, Y, L$ be as in the condition (7) of 3.2. Define $\varphi_{0}(f)=$ $\left(\varphi_{0}\left(f_{\lambda}\right)\right)_{\lambda \in \Lambda} \in I_{\Lambda}\left(\mathbf{R}^{n}\right)$. Then there is an open cover $\left(\Lambda_{j}\right)$ of $\Lambda$, consisting only of $\Lambda$ in the case (c), such that $\varphi_{0}(f) \mid Y \times \Lambda_{j}$ is for each $j$, respectively, (a) BL or (b), (c) $L_{0}-\mathrm{BL}$ with $L_{0}$ depending only on $L$ and $n$.

(4) Let $h, Y, \eta$ be as in the condition (8) of 3.2, with $\operatorname{dim} Y \geq 2$ in (b). Then $\varphi_{0}(h) \mid Y$ is, respectively, (a) QS or (b), (c) $\eta_{0}-\mathrm{QS}$ with $\eta_{0}$ depending only on $\eta$ and $n$.

The proof of Lemma 4.11 will be finished in 4.14.

4.12. Constructions in the case $m \geq 1$. Let $k=n-m$. Choose a point $q \in Q^{m} \backslash \pi_{0} \bar{D}^{m}$ and a LIP immersion $\alpha_{0}: Q^{m} \backslash q \rightarrow 4 B^{m}$. By the LIP Schoenflies theorem [LV, 7.7], we may assume that $\alpha_{0} \pi_{0}=$ id on a neighbourhood of $\bar{D}^{m}$. Set $\alpha=\alpha_{0} \times$ id: $\left(Q^{m} \backslash q\right) \times \mathbf{R}^{k} \rightarrow \mathbf{R}^{n}=\mathbf{R}^{m} \times \mathbf{R}^{k}$. Then $\alpha e=v$ on a neighbourhood of $v^{-1}\left[\bar{D}^{m} \times \widetilde{B}^{k}\right]$ in $A_{k}^{n}$. Let $R=\frac{1}{9} \min \left(r_{m}, \sigma_{Q}\left(q, \pi_{0} \bar{D}^{m}\right)\right)$, and set $D_{j}=$ $B_{\sigma_{Q}}(q, j R)$ for $1 \leq j \leq 8$; then $\bar{D}_{j} \cap \pi_{0} \bar{D}^{m}=\emptyset$ for each $j$.

We construct open sets $V_{1}, \ldots, V_{8}$ in $Q^{m} \times \mathbf{R}^{k}$, a neighbourhood $\mathcal{P}$ of id in $E\left(U, A^{\prime} ; X\right)$, and for each $i \in\{1, \ldots, 8\}$, considering $E\left(V_{i} ; Q^{m} \times \mathbf{R}^{k}\right)$ with the uniform topology induced by $\varrho$, a continuous map $\varphi_{i}: \mathcal{P} \rightarrow E\left(V_{i} ; Q^{m} \times \mathbf{R}^{k}\right)$ with $\varphi_{i}(\mathrm{id})=\mathrm{id}$, such that the following five conditions are satisfied, where $h \in \mathcal{P}$ and $g_{i}=\varphi_{i}(h)(1 \leq i \leq 8)$ :

(1) $\alpha g_{1}=h \alpha \mid V_{1}$, and $g_{i}=g_{1}$ on $\pi B$ for $i=2,4,6,8$.

(2) If $h$ respects $Y \in \mathcal{Y}$, then $g_{i}$ respects $Y^{*}$.

(3) If $h, h^{\prime} \in \mathcal{P}$ respect $Y \in \mathcal{Y}$ and $h=h^{\prime}$ on $U \cap Y$, then $\varphi_{i}(h)=\varphi_{i}\left(h^{\prime}\right)$ on $V_{i} \cap Y^{*}$.

(4) In the situation of 4.11(3) and defining $\varphi_{i}(f)=\left(\varphi_{i}\left(f_{\lambda}\right)\right)_{\lambda \in \Lambda} \in I_{\Lambda}\left(V_{i}\right.$; $\left.Q^{m} \times \mathbf{R}^{k}\right)$, we have that $\varphi_{i}(f) \mid\left(V_{i} \cap Y^{*}\right) \times \Lambda$ is, respectively, (a) LIP or (b) locally $L_{i}$-BL or (c) $L_{i}$-BL with $L_{i}$ depending on $(L, n, i)$ only.

(5) In the situation of 4.11(4), $g_{i} \mid V_{i} \cap Y^{*}$ is, respectively, (a) LQS or (b) locally $\eta_{i}-\mathrm{QS}$ or (c) $\eta_{i}-\mathrm{QS}$ with $\eta_{i}$ depending on $(\eta, n, i)$ only.

These properties will usually not be explicitly mentioned again as the construction proceeds.

The maps $\varphi_{1}, \ldots, \varphi_{8}$ will be constructed consecutively. We construct $\mathcal{P}$ setting first $\mathcal{P}=E\left(U, A^{\prime} ; X\right)$ and replacing then $\mathcal{P}$ gradually by smaller neighbourhoods, again denoted by $\mathcal{P}$, whenever need arises.

Choosing $\mathcal{P}$ sufficiently small, we may assume that $\varepsilon_{i}=\sup \left\{\varrho\left(g_{i}\right.\right.$, id $) \mid h \in$ $\mathcal{P}\}$ for $1 \leq i \leq 8$ is as small as needed in the constructions. 
Thus, let $h \in \mathcal{P}$. We first construct

$$
g_{1}: V_{1}=\left(Q^{m} \backslash \bar{D}_{2}\right) \times 4 B^{p-m} \times 4 B^{n-p} \rightarrow\left(Q^{m} \backslash \bar{D}_{1}\right) \times \mathbf{R}^{k}
$$

with $g_{1}(x, y, z)=(x, y, z)$ whenever $|y| \geq \frac{1}{2}$ as follows. Observing that $\bar{V}_{1}$ is compact (in $Q^{m} \times \mathbf{R}^{k}$ ), choose a cover $\left\{W_{1}, \ldots, W_{s}\right\}$ of $\bar{V}_{1}$ by open subsets of $\left(Q^{m} \backslash \bar{D}_{1}\right) \times \mathbf{R}^{k}$ such that $\alpha \mid W_{i}$ is BL for each $i$ and such that $\alpha \mid W_{i} \cup W_{j}$ is injective whenever $W_{i} \cap W_{j} \neq \emptyset$. Then choose an open cover $\left\{G_{1}, \ldots, G_{s}\right\}$ of the space $V_{1}$ with a positive Lebesgue number such that $\bar{G}_{i} \subset W_{i}$ for each $i$. Finally, choose $\mathcal{P}$ so small that $h \alpha \bar{G}_{i} \subset \alpha W_{i}$ for each $i$. Now set $g_{1}\left|G_{i}=\left(\alpha \mid W_{i}\right)^{-1} h \alpha\right| G_{i}$ for $1 \leq i \leq s$. Then $g_{1}$ is well-defined. As injective, $g_{1}$ is an embedding. For $\varepsilon_{1}$ small enough, (4c) and (5c) follow from 2.4 or 2.5 , respectively.

We let $V_{2}=\left(Q^{m} \times 4 B^{p-m} \backslash \bar{D}_{2} \times \bar{B}^{p-m}(0.6)\right) \times 4 B^{n-p}$ and define $g_{2}: V_{2} \rightarrow$ $Q^{m} \times \mathbf{R}^{k}$ by

$$
g_{2}=g_{1} \cup\left(\mathrm{id} \mid Q^{m} \times\left(4 B^{p-m} \backslash \bar{B}^{p-m}(0.6)\right) \times 4 B^{n-p}\right) .
$$

Assuming $\varepsilon_{1}<0.1$ we get an embedding. For $\varepsilon_{1}$ small enough, (4c) and (5c) follow from 2.4 or 2.5 , respectively.

We construct a self-homeomorphism $g_{3}$ of $V_{3}=\widehat{Q}$ using 4.9. Lemma 4.9 implies $\mathcal{D}^{*}\left(\widehat{Q} ; A_{1}, A_{1}^{\prime}, U_{1}, B_{1}, B_{1}^{\prime} ; \mathcal{Y}^{*} \mid \widehat{Q}\right)$, where

$$
\begin{aligned}
& A_{1}=Q^{m} \times\left(\mathbf{R}^{p-m} \backslash B^{p-m}(0.7)\right) \times\left(\mathbf{R}^{n-p} \backslash 0\right), \\
& A_{1}^{\prime}=Q^{m} \times\left(\mathbf{R}^{p-m} \backslash B^{p-m}(0.61)\right) \times\left(\mathbf{R}^{n-p} \backslash 0\right), \\
& U_{1}=\left(Q^{m} \times 4 B^{p-m} \backslash \bar{D}_{2} \times \bar{B}^{p-m}(0.6)\right) \times\left(4 B^{n-p} \backslash \bar{B}^{n-p}(0.7)\right) \subset V_{2}, \\
& B_{1}=\left(Q^{m} \times \bar{B}^{p-m}(3.1) \backslash D_{4} \times B^{p-m}(0.62)\right) \times\left(\bar{B}^{n-p}(3.1) \backslash B^{n-p}(0.9)\right), \\
& B_{1}^{\prime}=\left(Q^{m} \times \bar{B}^{p-m}(3.2) \backslash D_{3} \times B^{p-m}(0.61)\right) \times\left(\bar{B}^{n-p}(3.2) \backslash B^{n-p}(0.8)\right) .
\end{aligned}
$$

Let $\mathcal{P}_{1}$ be the neighbourhood of id in $E\left(U_{1}, A_{1}^{\prime} ; \widehat{Q}\right)$ and $\psi_{1}: \mathcal{P}_{1} \times I \rightarrow H(\widehat{Q})$ the continuous map given by the above deformation statement. Assuming $\varepsilon_{2}$ to be sufficiently small, we have that $g_{2} U_{1} \subset \widehat{Q}$ and $g_{2} \mid U_{1} \in \mathcal{P}_{1}$. Then we define $g_{3}=\psi_{1}\left(g_{2} \mid U_{1}, 1\right)$. It follows that $g_{3}=$ id on $A_{1} \cup\left(\widehat{Q} \backslash B_{1}^{\prime}\right)$ and $g_{3}=g_{2}$ on $B_{1}$.

We next construct

$$
g_{4}: V_{4}=Q^{m} \times 3 B^{p-m} \times 3 B^{n-p} \backslash \bar{D}_{5} \times \bar{B}^{p-m}(0.7) \times \bar{B}^{n-p} \rightarrow Q^{m} \times \mathbf{R}^{k}
$$

with $g_{4}=\mathrm{id}$ on $Q^{m} \times\left(3 B^{p-m} \backslash \bar{B}^{p-m}(0.7)\right) \times 3 B^{n-p}$. Define open subsets

$$
\begin{aligned}
& N_{2}=\left(Q^{m} \times 3 B^{p-m} \backslash \bar{D}_{5} \times \bar{B}^{p-m}(0.7)\right) \times 3 B^{n-p}, \\
& N_{3}=Q^{m} \times 3 B^{p-m} \times\left(3 B^{n-p} \backslash \bar{B}^{n-p}\right)
\end{aligned}
$$


of $Q^{m} \times \mathbf{R}^{k}$. Then $V_{4}=N_{2} \cup N_{3}, \bar{N}_{i}$ is compact in $V_{i}$ for $i=2,3$, and $\bar{N}_{2} \cap \bar{N}_{3} \subset$ int $B_{1}$. Thus, we can set $g_{4}=\left(g_{2} \mid N_{2}\right) \cup\left(g_{3} \mid N_{3}\right)$. Assuming $\varepsilon_{2}$ and $\varepsilon_{3}$ to be sufficiently small, $g_{4}$ is an embedding, and (4c) and (5c) follow from 2.4 or 2.5 , respectively.

We construct $g_{5}: V_{5}=D_{7} \times B^{p-m}(0.81) \times B^{n-p}(2.5) \rightarrow Q^{m} \times \mathbf{R}^{k}$ with $g_{5}=g_{4}$ outside $D_{6} \times B^{p-m}(0.8) \times 2 B^{n-p}$ by applying 2.13 , together with 2.23 for (5c) and 2.2 for (5b), to the embedding of $V_{5} \backslash \bar{D}_{5} \times \bar{B}^{p-m}(0.7) \times \bar{B}^{n-p}$ into $D_{8} \times B^{p-m} \times 3 B^{n-p}$ defined by $g_{4}$. This is possible if $\varepsilon_{4}$ is small enough by the aid of an auxiliary BL homeomorphism.

We define $g_{6}: V_{6}=Q^{m} \times 3 B^{p-m} \times 3 B^{n-p} \rightarrow Q^{m} \times \mathbf{R}^{k}$ with $g_{6}=$ id on $Q^{m} \times\left(3 B^{p-m} \backslash B^{p-m}(0.8)\right) \times 3 B^{n-p}$ setting

$$
g_{6}=\left(g_{4} \mid V_{6} \backslash \bar{D}_{6} \times \bar{B}^{p-m}(0.8) \times 2 \bar{B}^{n-p}\right) \cup g_{5}
$$

and assuming that $\varepsilon_{4}$ and $\varepsilon_{5}$ are sufficiently small. Again, (4c) and (5c) follow from 2.4 or 2.5 , respectively.

We construct a self-homeomorphism $g_{7}$ of $V_{7}=\widehat{Q}$ using 4.9 , which allows us to apply $\mathcal{D}^{*}\left(\widehat{Q} ; A_{2}, A_{2}^{\prime}, U_{2}, B_{2}, B_{2}^{\prime} ; \mathcal{Y}^{*} \mid \widehat{Q}\right)$, where

$$
\begin{aligned}
& A_{2}=Q^{m} \times\left(\mathbf{R}^{p-m} \backslash B^{p-m}(0.9)\right) \times\left(\mathbf{R}^{n-p} \backslash 0\right), \\
& A_{2}^{\prime}=Q^{m} \times\left(\mathbf{R}^{p-m} \backslash B^{p-m}(0.8)\right) \times\left(\mathbf{R}^{n-p} \backslash 0\right), \\
& U_{2}=Q^{m} \times 3 B^{p-m} \times\left(B^{n-p} \backslash \bar{B}^{n-p}\left(\frac{1}{5}\right)\right), \\
& B_{2}=Q^{m} \times \bar{B}^{p-m} \times\left(\bar{B}^{n-p}\left(\frac{1}{2}\right) \backslash B^{n-p}\left(\frac{1}{3}\right)\right), \\
& B_{2}^{\prime}=Q^{m} \times 2 \bar{B}^{p-m} \times\left(\bar{B}^{n-p}(0.9) \backslash B^{n-p}\left(\frac{1}{4}\right)\right) .
\end{aligned}
$$

Let $\mathcal{P}_{2}$ be the neighbourhood of id in $E\left(U_{2}, A_{2}^{\prime} ; \widehat{Q}\right)$ and $\psi_{2}: \mathcal{P}_{2} \times I \rightarrow H(\widehat{Q})$ the continuous map thus obtained. Assuming $\varepsilon_{6}$ to be sufficiently small, we have that $g_{6} U_{2} \subset \widehat{Q}$ and $g_{6} \mid U_{2} \in \mathcal{P}_{2}$. Then we define $g_{7}=\psi_{2}\left(g_{6} \mid U_{2}, 1\right)$. It follows that $g_{7}=$ id on $A_{2} \cup\left(\widehat{Q} \backslash B_{2}^{\prime}\right)$ and $g_{7}=g_{6}$ on $B_{2}$.

We finally construct a self-homeomorphism $g_{8}$ of $V_{8}=Q^{m} \times \mathbf{R}^{k}$ setting $g_{8}=g_{6}$ on $Q^{m} \times B^{p-m} \times B^{n-p}\left(\frac{1}{2}\right), g_{8}=g_{7}$ on $Q^{m} \times B^{p-m} \times\left(B^{n-p} \backslash \bar{B}^{n-p}\left(\frac{1}{3}\right)\right)$, and $g_{8}=$ id outside $Q^{m} \times \bar{B}^{p-m}(0.9) \times \bar{B}^{n-p}(0.9)$, and assuming that $\varepsilon_{6}$ and $\varepsilon_{7}$ are small enough. To verify (5c), we first apply 2.5 for $g_{8} \mid\left(Q^{m} \times B^{p-m} \times B^{n-p}\right) \cap Y^{*}$ and then 2.9. Similarly, to verify (4c), we first apply 2.4 and then 2.7 .

4.13. Constructions in the case $m=0$. We modify 4.12. Let $V_{6}=U$, $V_{7}=\widehat{R}=\mathbf{R}^{n} \backslash \mathbf{R}^{p}$, and $V_{8}=\mathbf{R}^{n}$. For a sufficiently small neighbourhood $\mathcal{P}$ of id in $E\left(U, A^{\prime} ; X\right)$ and for each $i=6,7,8$, we construct a continuous map $\varphi_{i}: \mathcal{P} \rightarrow E\left(V_{i} ; \mathbf{R}^{n}\right)$ with $\varphi_{i}(\mathrm{id})=$ id such that the conditions $(2)-(5)$ in 4.12 are satisfied, where only $Q^{m} \times \mathbf{R}^{k}$ is replaced by $\mathbf{R}^{n}$ and $Y^{*}$ by $Y$. We set $g_{6}=\varphi_{6}(h)=h$. We then construct $g_{7}=\varphi_{7}(h) \in H(\widehat{R})$ as in 4.12 using 
$\mathcal{D}^{*}\left(\widehat{R} ; A_{2}, A_{2}^{\prime}, U_{2}, B_{2}, B_{2}^{\prime} ; \mathcal{Y} \mid \widehat{R}\right)$, where the sets $A_{2}, A_{2}^{\prime}, \ldots$ are as in 4.12 (delete $\left.Q^{m}\right)$ and which follows from the hypothesized $\mathcal{D}(\widehat{R} ; \mathcal{Y} \mid \widehat{R})$ by 3.9 . We finally construct $g_{8}=\varphi_{8}(h) \in H\left(\mathbf{R}^{n}\right)$ as in 4.12 (delete $\left.Q^{m}\right)$. Then $g_{8}=h$ on $B$ and $g_{8}=$ id outside $B^{p} \times B^{n-p}$.

4.14. Proof of 4.11. Suppose first that $m \geq 1$. Let $\mathcal{P}$ be as constructed in 4.12. If $h \in \mathcal{P}$, then $g_{8}$ restricts to a homeomorphism $g_{9} \in H\left(Q^{*}, \partial Q^{*}\right)$. Assuming that $\varepsilon_{8}$ is sufficiently small, we can define $g=\varphi_{0}(h)$ as the homeomorphism $\bar{g}_{9}$ given by 4.6 and 4.7 . We prove (1). There is a neighbourhood $B_{0}$ of $B$ in $B^{*}$ such that $\alpha e=v$ on $v^{-1} B_{0}$; see 4.12. Choosing $\mathcal{P}$ small enough, we have that $g v^{-1} B \subset v^{-1} B_{0}$. Then, by 4.12(1), $h v=h \alpha e=\alpha g_{1} e=\alpha g_{9} e=\alpha e g=v g$ on $v^{-1} B$.

Suppose $m=0$. Now $v$ is the homeomorphism $\tau_{0} \mid \bar{B}^{n}: \bar{B}^{n} \rightarrow \bar{B}^{p} \times \bar{B}^{n-p}$. Let $\mathcal{P}$ be as constructed in 4.13. Then we can define $\varphi_{0}(h)=\tau_{0}^{-1} g_{8} \tau_{0}$. For (3a) and (3b), cf. the proof of 4.7. For (4a), use 2.9. For (4b), use 2.3. व

4.15. Proof of 4.2. It is easy to find a BL homeomorphism $w: \mathbf{R}^{n} \rightarrow$ $\mathbf{R}^{n}$ which respects each $Y \in \mathcal{Y}_{n}$ and for which $w=u$ on $u^{-1}\left[\bar{B}^{m}\left(\frac{1}{2}\right) \times \bar{B}^{k}\right]$ and $w B^{n} \subset B^{m}\left(\frac{3}{4}\right) \times B^{k}$. Then also $\bar{w}=\left(\mathrm{id} \times \tau_{0}\right) w: \mathbf{R}^{n} \rightarrow \mathbf{R}^{n}$ is a BL homeomorphism respecting each $Y \in \mathcal{Y}_{n}$. Let $\varphi_{0}: \mathcal{P} \rightarrow \mathcal{H}=H\left(\mathbf{R}^{n}, \mathbf{R}^{n} \backslash\right.$ $B^{n}$ ) and $\psi: \mathcal{H} \times I \rightarrow \mathcal{H}$ be the maps given by 4.11 or 2.24 , respectively. Set $\varphi(h, t)=\bar{w} \psi\left(\varphi_{0}(h), t\right)^{-1} \bar{w}^{-1} h$ for $h \in \mathcal{P}, t \in I$. Then $\varphi$ is a continuous map $\mathcal{P} \times I \rightarrow E(U, A ; X)$ satisfying the conditions (1), (2), and (5)-(8) of 3.2. Choosing $\mathcal{P}$ so small that $\bar{w} B^{n} \subset h B^{\prime}$ for each $h \in \mathcal{P}$ yields 3.2(4). Let $\widetilde{B}=\bar{B}^{m}\left(\frac{1}{2}\right) \times \bar{B}^{p-m} \times \bar{B}^{n-p}$. Since $\bar{D}^{m} \subset B^{m}\left(\frac{1}{2}\right)$ if $m \geq 1$, choosing $\mathcal{P}$ sufficiently small implies that $h B \subset \widetilde{B}$ whenever $h \in \mathcal{P}$. We can now verify 3.2(3). Note that $\bar{w}=v$ on $v^{-1} \widetilde{B}$. Let $h \in \mathcal{P}$ and $g=\varphi_{0}(h)$. Since $\psi(g, 1)=g$, using 4.11(1) we see that $\varphi(h, 1)=\bar{w} g^{-1} \bar{w}^{-1} h=\bar{w} g^{-1} v^{-1} h=\bar{w} v^{-1}=$ id on $B$. ㅁ

\section{Basic deformation property of $\mathbf{R}^{n}$ and of $\mathbf{R}_{+}^{n}$}

In this section we prove that the statements $\mathcal{D}\left(\mathbf{R}^{n} ; \mathcal{Y}_{n}\right)$ and $\mathcal{D}\left(\mathbf{R}_{+}^{n} ; \mathcal{Y}_{n}^{+}\right)$are true.

5.1. Lemma. Let $0 \leq p \leq n \geq 1$ be integers, let $\mathcal{Y}=\mathcal{Y}_{n p}$, suppose that $\mathcal{D}\left(\mathbf{R}^{n} \backslash \mathbf{R}^{p} ; \mathcal{Y} \mid \mathbf{R}^{n} \backslash \mathbf{R}^{p}\right)$ holds, and let $X, A, A^{\prime}, U, B, B^{\prime}$ be as in 3.2 with $X=\mathbf{R}^{n}$ and $B \subset \mathbf{R}^{p}$. Then there is a compact set $B_{0} \subset \subset B^{\prime}$ with $B \subset \subset B_{0}$ such that $\mathcal{D}\left(\mathbf{R}^{n} ; A, A^{\prime}, U, B_{0}, B^{\prime} ; \mathcal{Y}\right)$ holds.

Proof. Let $T$ be a rectilinear triangulation of $\mathbf{R}^{p}$. Let $T^{\prime}$ be the first barycentric subdivision of $T$ and $T^{\prime \prime}$ the second one. For a simplex $\sigma \in T$, let $\hat{\sigma}$ be the barycentre of $\sigma, H_{\sigma}=\bigcup\left\{\tau \mid \hat{\sigma} \in \tau \in T^{\prime \prime}\right\}$, and $i\left(H_{\sigma}\right)=\operatorname{dim} \sigma$. Then $\mathcal{H}=$ $\left\{H_{\sigma} \mid \sigma \in T\right\}$ is a handle decomposition of $\mathbf{R}^{p}$ as in [RS, p. 82]. In fact, if $\sigma \in T$, $P_{\sigma}=\bigcup\left\{H_{\tau} \mid \tau \in T, \operatorname{dim} \tau<\operatorname{dim} \sigma\right\}, \widetilde{H}_{\sigma}=\operatorname{cl}\left(\bigcup\left\{\tau \mid \hat{\sigma} \in \tau \in T^{\prime}\right\} \backslash P_{\sigma}\right)$, and 
$m=p-i\left(H_{\sigma}\right)$, it is easy to show that $\left(\widetilde{H}_{\sigma}, H_{\sigma}, \widetilde{H}_{\sigma} \cap P_{\sigma}\right)$ is PL homeomorphic to $\left(2 I^{m} \times I^{p-m}, I^{m} \times I^{p-m}, 2 I^{m} \times \partial I^{p-m}\right)$. Setting $H_{\sigma} \leq H_{\tau}$ if $H_{\sigma} \cap H_{\tau} \neq \emptyset$ and $i\left(H_{\sigma}\right) \leq i\left(H_{\tau}\right)$ (i.e., if $\sigma$ is a face of $\tau$ ) we obtain a partial order for $\mathcal{H}$. Let $\mathcal{H}_{0}$ and $\mathcal{H}_{1}$, respectively, be the sets of the handles $H \in \mathcal{H}$ such that there is $H^{\prime} \in \mathcal{H}$ with $H \leq H^{\prime}$ and with either $H^{\prime} \cap A \neq \emptyset$ or $H^{\prime} \cap B \neq \emptyset$, respectively. Then $H \leq H_{i} \in \mathcal{H}_{i}$ implies $H \in \mathcal{H}_{i}$ for $i=0,1$. Choosing $T$ fine enough we may assume that $\bigcup \mathcal{H}_{0} \subset \subset A^{\prime}$ and $\bigcup \mathcal{H}_{1} \subset \subset B^{\prime}$. Let $\mathcal{H}_{1} \backslash \mathcal{H}_{0}$ consist of $s$ handles $H_{1}, \ldots, H_{s}$ with $i\left(H_{j}\right) \leq i\left(H_{j+1}\right)$ for all $j<s$. Let $\mathcal{H}_{j}^{\prime}=\left(\mathcal{H}_{0} \cap \mathcal{H}_{1}\right) \cup\left\{H_{1}, \ldots, H_{j}\right\}$ and $P_{j}=\bigcup \mathcal{H}_{j}^{\prime}$ for $0 \leq j \leq s$. Then $P_{s}=\bigcup \mathcal{H}_{1}$ is a neighbourhood of $B$ in $\mathbf{R}^{p}$. For each $H \in \mathcal{H}$ with $H \cap H_{j} \neq \emptyset$ we have that $i(H)<i\left(H_{j}\right)$ if and only if $H \in \mathcal{H}_{j-1}^{\prime}$.

Choose an open neighbourhood $V_{0} \subset \subset A^{\prime} \cap B^{\prime}$ of $P_{0}$ in $\mathbf{R}^{p}$. Then, inductively, for each $j \in\{1, \ldots, s\}$ we can choose a PL embedding $\alpha_{j}: 6 I^{p} \rightarrow$ (int $\left.B^{\prime} \backslash A\right) \cap \mathbf{R}^{p}$ and define an open neighbourhood $V_{j} \subset \subset B^{\prime}$ of $P_{j}$ in $\mathbf{R}^{p}$ such that, setting $m=p-i\left(H_{j}\right)$, we have that

$$
\begin{gathered}
\alpha_{j}\left[I^{m}\left(\frac{1}{3}\right) \times 2 I^{p-m}\right]=H_{j}, \\
\alpha_{j}^{-1} P_{j-1}=6 I^{m} \times\left(6 I^{p-m} \backslash 2 J^{p-m}\right), \\
\alpha_{j}\left[6 I^{m} \times\left(6 I^{p-m} \backslash J^{p-m}\left(\frac{1}{2}\right)\right)\right] \subset V_{j-1}, \\
V_{j}=\left(V_{j-1} \backslash \alpha_{j}\left[6 I^{m} \times I^{p-m}\right]\right) \cup \alpha_{j}\left[J^{m}\left(\frac{1}{2}\right) \times 6 J^{p-m}\right] .
\end{gathered}
$$

Choose positive numbers $\varepsilon_{0}>\varepsilon_{1}^{\prime}>\varepsilon_{1}>\cdots>\varepsilon_{s}^{\prime}>\varepsilon_{s}$ such that $V_{0} \times$ $B^{n-p}\left(\varepsilon_{0}\right) \subset \subset A^{\prime} \cap B^{\prime}$ and $\alpha_{j}\left[6 I^{p}\right] \times \bar{B}^{n-p}\left(\varepsilon_{0}\right) \subset \operatorname{int} B^{\prime} \backslash A$ for $1 \leq j \leq s$. We show that the compact neighbourhood $B_{0}=P_{s} \times \bar{B}^{n-p}\left(\frac{1}{2} \varepsilon_{s}\right)$ of $B$ in int $B^{\prime}$ is the desired set.

For $1 \leq j \leq s$, choose a radial $\mathrm{BL}$ homeomorphism $\beta_{j}: 6 \bar{B}^{n-p} \rightarrow \bar{B}^{n-p}\left(\varepsilon_{j}^{\prime}\right)$ with $\beta_{j} \bar{B}^{n-p}\left(\frac{1}{2}\right)=\bar{B}^{n-p}\left(\varepsilon_{j}\right)$. Then $\gamma_{j}=\alpha_{j} \times \beta_{j}: 6 I^{p} \times 6 \bar{B}^{n-p} \rightarrow B^{\prime} \backslash A$ is a BL embedding, which respects each $Y \in \mathcal{Y}$. Let $W_{j}=V_{j} \times B^{n-p}\left(\varepsilon_{j}\right)$ for $j \geq 0$. If $j \geq 1$, let $m$ be as above, and let

$$
\begin{aligned}
& X_{j}=\gamma_{j}\left[6 J^{p} \times 6 B^{n-p}\right], \\
& A_{j}=\gamma_{j}\left[5 I^{m} \times\left(5 I^{p-m} \backslash J^{p-m}\right) \times 5 \bar{B}^{n-p}\right], \\
& A_{j}^{\prime}=\gamma_{j}\left[6 I^{m} \times\left(6 I^{p-m} \backslash J^{p-m}\left(\frac{1}{2}\right)\right) \times 6 \bar{B}^{n-p}\right], \\
& U_{j}=\gamma_{j}\left[5 J^{p} \times 5 B^{n-p}\right], \\
& B_{j}=\gamma_{j}\left[I^{m}\left(\frac{1}{2}\right) \times I^{p-m} \times \bar{B}^{n-p}\left(\frac{1}{2}\right)\right], \\
& B_{j}^{\prime}=\gamma_{j}\left[I^{m} \times 2 I^{p-m} \times 2 \bar{B}^{n-p}\right] .
\end{aligned}
$$

Then $\mathcal{D}\left(\mathbf{R}^{n} \backslash \mathbf{R}^{p} ; \mathcal{Y} \mid \mathbf{R}^{n} \backslash \mathbf{R}^{p}\right)$ and 4.2 imply, by 3.5 .2 , that $\mathcal{D}_{1}\left(X_{j} ; A_{j}, A_{j}^{\prime} \cap X_{j}\right.$, $\left.U_{j}, B_{j}, B_{j}^{\prime} ; \mathcal{Y} \mid X_{j}\right)$ holds. By 3.8 it follows that $\mathcal{D}\left(\mathbf{R}^{n} ; A_{j}, A_{j}^{\prime}, U, B_{j}, B_{j}^{\prime} ; \mathcal{Y}\right)$ holds yielding a set $\mathcal{P}_{j}$ and a map $\varphi_{j}$. Note that $A_{j}^{\prime} \subset W_{j-1}, W_{j} \cap B_{j}^{\prime} \subset A_{j} \cup B_{j}$, 
and $W_{j} \backslash B_{j}^{\prime} \subset W_{j-1}$. Thus, if $\mathcal{P}_{j}^{\prime}=\mathcal{P}_{j} \cap E\left(U, W_{j-1} ; \mathbf{R}^{n}\right)$, then $\varphi_{s}\left[\mathcal{P}_{s}^{\prime} \times 1\right] \subset$ $E\left(U, W_{s} ; \mathbf{R}^{n}\right)$ and we may assume that $\varphi_{j}\left[\mathcal{P}_{j}^{\prime} \times 1\right] \subset \mathcal{P}_{j+1}^{\prime}$ for $1 \leq j<s$. Since $W_{0} \subset A^{\prime}$ and $B_{0} \subset W_{s}$, we conclude by 3.10 that $\mathcal{P}=\mathcal{P}_{1}^{\prime} \cap E\left(U, A^{\prime} ; \mathbf{R}^{n}\right)$ is the desired set and $\varphi=\varphi_{s} * \cdots *\left(\varphi_{1} \mid \mathcal{P} \times I\right)$ the desired map satisfying $\mathcal{D}\left(\mathbf{R}^{n} ; A, A^{\prime}, U, B_{0}, B^{\prime} ; \mathcal{Y}\right)$.

5.2. Lemma. The analogue of 5.1 holds where $0 \leq p<n$ and $\mathcal{Y}=\mathcal{Y}_{n p}^{+}$and where each occurrence of $\mathbf{R}^{n}$ is replaced by $\mathbf{R}_{+}^{n}$.

Proof. In the proof of 5.1 replace $\mathbf{R}^{n}$ by $\mathbf{R}_{+}^{n}$ and $B^{n-p}(r)$ by $B^{n-p}(r) \cap \mathbf{R}_{+}^{n-p}$ for $r>0$; note that $\gamma_{j}$ respects $\mathbf{R}_{+}^{n}$. Apply 4.3 in place of 4.2 . $\square$

5.3. Lemma. The statement $\mathcal{D}\left(\mathbf{R}^{n} \backslash \mathbf{R}^{p} ; \mathcal{Y}_{n p} \mid \mathbf{R}^{n} \backslash \mathbf{R}^{p}\right)$ implies $\mathcal{D}\left(\mathbf{R}^{n} ; \mathcal{Y}_{n p}\right)$ whenever $0 \leq p \leq n \geq 1$.

Proof. We prove $\mathcal{D}=\mathcal{D}\left(\mathbf{R}^{n} ; A, A^{\prime}, U, B, B^{\prime} ; \mathcal{Y}_{n p}\right)$. Choose a closed set $A^{\prime \prime} \subset \subset$ $A^{\prime}$ with $A \subset \subset A^{\prime \prime}$. By 5.1 there is a compact set $B_{0} \subset \subset B^{\prime}$ with $B \cap \mathbf{R}^{p} \subset \subset B_{0}$ such that

$$
\mathcal{D}^{\prime}=\mathcal{D}\left(\mathbf{R}^{n} ; A^{\prime \prime}, A^{\prime}, U, B_{0}, B^{\prime} ; \mathcal{Y}_{n p}\right)
$$

holds.

Choose a compact set $B_{1} \subset \subset B_{0}$ with $B \cap \mathbf{R}^{p} \subset \subset B_{1}$. Then $B \backslash \operatorname{int} B_{1}$ is a compact set in $\mathbf{R}^{n} \backslash \mathbf{R}^{p}$; choose a compact neighbourhood $B^{\prime \prime} \subset B^{\prime}$ of it in $\mathbf{R}^{n} \backslash \mathbf{R}^{p}$. Choose an open neighbourhood $U_{1}$ of $B^{\prime \prime}$ with $\bar{U}_{1}$ compact in $U \backslash \mathbf{R}^{p}$. From $\mathcal{D}\left(\mathbf{R}^{n} \backslash \mathbf{R}^{p} ; \mathcal{Y}_{n p} \mid \mathbf{R}^{n} \backslash \mathbf{R}^{p}\right)$ it follows that

$$
\mathcal{D}_{1}\left(\mathbf{R}^{n} ; A \cup B_{1}, A^{\prime \prime} \cup B_{0}, U_{1}, B \backslash \operatorname{int} B_{1}, B^{\prime \prime} ; \mathcal{Y}_{n p}\right)
$$

holds (see 3.5.2). By 3.8 we can drop here the subscript 1 from $\mathcal{D}_{1}$ and from $U_{1}$. Thus,

$$
\mathcal{D}^{\prime \prime}=\mathcal{D}\left(\mathbf{R}^{n} ; A \cup B_{1}, A^{\prime \prime} \cup B_{0}, U, B, B^{\prime} ; \mathcal{Y}_{n p}\right)
$$

holds. But $\mathcal{D}^{\prime}$ and $\mathcal{D}^{\prime \prime}$ imply $\mathcal{D}$ by 3.10. $\square$

5.4. Lemma. The statement $\mathcal{D}\left(\mathbf{R}_{+}^{n} \backslash \mathbf{R}^{p} ; \mathcal{Y}_{n p}^{+} \mid \mathbf{R}_{+}^{n} \backslash \mathbf{R}^{p}\right)$ implies $\mathcal{D}\left(\mathbf{R}_{+}^{n} ; \mathcal{Y}_{n p}^{+}\right)$ whenever $0 \leq p<n$.

Proof. In the proof of 5.3 , replace $\left(\mathbf{R}^{n}, \mathcal{Y}_{n p}\right)$ by $\left(\mathbf{R}_{+}^{n}, \mathcal{Y}_{n p}^{+}\right)$, and apply 5.2 in place of 5.1. ㅁ

5.5. Theorem. The statement $\mathcal{D}\left(\mathbf{R}^{n} ; \mathcal{Y}_{n}\right)$ holds for each $n \geq 1$.

Proof. For $0 \leq p \leq n$, let $\mathcal{Y}_{n}^{(p)}$ be the family of the sets $Y=Y_{1} \times \cdots \times Y_{n} \in \mathcal{Y}_{n}$ such that $p$ of the factors $Y_{i} \subset \mathbf{R}^{1}$ are of the form $\mathbf{R}_{+}^{1}$ or $\mathbf{R}_{-}^{1}$ and the rest of the form $\{0\}$. For such a set $Y \in \mathcal{Y}_{n}^{(p)}$, define an open set $Y^{*}=Y_{1}^{*} \times \cdots \times Y_{n}^{*}$ in $\mathbf{R}^{n}$ letting $Y_{i}^{*}=\operatorname{Int} Y_{i}$ if $Y_{i}=\mathbf{R}_{+}^{1}$ or $\mathbf{R}_{-}^{1}$ and letting $Y_{i}^{*}=\mathbf{R}^{1}$ otherwise. 
Let $\mathcal{Z}=\left\{Z \mid Z \subset \mathbf{R}^{n}\right.$ open, $\mathcal{D}\left(Z ; \mathcal{Y}_{n} \mid Z\right)$ holds $\}$. We show by induction on $n-p$ that $\left\{Y^{*} \mid Y \in \mathcal{Y}_{n}^{(p)}\right\} \subset \mathcal{Z}$ for each $p, 0 \leq p \leq n$. Consider $Y \in \mathcal{Y}_{n}^{(p)}$ as above. Then $Y^{*} \backslash Y=\bigcup\left\{Y^{*} \mid Y \subset Y^{\prime} \in \mathcal{Y}_{n}^{(p+1)}\right\}$ if $p<n$. Hence, it follows from the obvious validity of $\mathcal{D}(\emptyset ;\{\emptyset\})$ if $p=n$ or from the inductive hypothesis and 3.11(ii) if $p<n$ that $Y^{*} \backslash Y \in \mathcal{Z}$. Let $g_{i}: Y_{i}^{*} \rightarrow \mathbf{R}^{1}$ for $1 \leq i \leq n$ be a LIP homeomorphism. Then, for a suitable orthogonal map $\sigma$ of $\mathbf{R}^{n}$ permuting the standard basis of $\mathbf{R}^{n}$, we get a LIP homeomorphism $g=\sigma\left(g_{1} \times \cdots \times g_{n}\right): Y^{*} \rightarrow \mathbf{R}^{n}$ for which $g\left[Y^{*} \cap Y\right]=\mathbf{R}^{p}$. Now note that $Y^{\prime} \in \mathcal{Y}_{n}$ meets $Y^{*}$ if and only if $Y \subset Y^{\prime}$. Hence, $g$ carries the family $\left(\mathcal{Y}_{n} \mid Y^{*}\right) \backslash\{\emptyset\}$ onto $\mathcal{Y}_{n p}$. Thus, $Y^{*} \in \mathcal{Z}$ by 3.13 and 5.3. This completes the induction.

The case $p=0$ then implies that $\mathbf{R}^{n} \in \mathcal{Z}$. $\square$

5.6. Theorem. The statement $\mathcal{D}\left(\mathbf{R}_{+}^{n} ; \mathcal{Y}_{n}^{+}\right)$holds for each $n \geq 1$.

Proof. Modify the proof of 5.5 as follows. Replace $\mathcal{Y}_{n}$ throughout by $\mathcal{Y}_{n}^{+}$. Excluding the definition of $g$ whenever $Y_{n}=\mathbf{R}_{+}^{1}$, replace $\mathbf{R}^{n}$ by $\mathbf{R}_{+}^{n}$. If $Y_{n}=$ $\{0\}$, let $Y_{n}^{*}=\mathbf{R}_{+}^{1}$ and $g_{n}=$ id: $Y_{n}^{*} \rightarrow \mathbf{R}_{+}^{1}$, replace $\mathcal{Y}_{n p}$ by $\mathcal{Y}_{n p}^{+}$, and apply 5.4 in place of 5.3.

5.7. Corollary. The statements $\mathcal{D}^{*}\left(\mathbf{R}^{n} ; \mathcal{Y}_{n}\right)$ and $\mathcal{D}^{*}\left(\mathbf{R}_{+}^{n} ; \mathcal{Y}_{n}^{+}\right)$hold for each $n \geq 1$.

Proof. 5.5, 5.6, and 3.9. व

5.8. Remark. (This remark and a similar one, 6.3 , are only needed in order that in 7.2 the set $\partial N$ could be included in $\mathcal{Y}$.) In the setting of $\mathcal{D}\left(\mathbf{R}^{n} ; \mathcal{Y}_{n}\right)$ in 5.5 , we can add the modifications of the conditions (7) and (8) of 3.2 to the list of 3.2 in which $Y$ is given as the union of two arbitrary sets $Y_{1}, Y_{2} \in \mathcal{Y}_{n}$ that are $l$-dimensional half-spaces for some $l \geq 1$ with $Y_{1} \cap Y_{2}=\partial Y_{1}=\partial Y_{2}$ and in which $f$ respects $Y_{1} \times \Lambda$ and $Y_{2} \times \Lambda$ and $h$ respects $Y_{1}$ and $Y_{2}$. For the modifications of (8a) and (8c) with $l=1$, i.e., for the condition $\left(8^{\prime}\right)$ in 4.4 .2 , this is seen from 4.4 .2 and the proofs of 5.1, 5.3, and 5.5. The other cases of the modified conditions easily follow from the respective original ones for $\mathcal{P}$ small enough (for (8) use 2.3 and $\left.\left[\mathrm{V} \ddot{a}_{1}, 35.1\right]\right)$.

A similar remark concerns 5.6 and 5.7.

5.9. Remark. In the settings of 5.5 and 5.6 , the condition ( $8 \mathrm{~b})$ of 3.2 can be strengthened to the form in which 'locally $\eta-\mathrm{QS}$ ' is replaced by ' $K-\mathrm{QC}$ ' and 'locally $\eta^{*}-\mathrm{QS}$ ' by ' $K^{*}-\mathrm{QC}$ where $K^{*}=c K^{\nu}$ with $c, \nu \geq 1$ independent of $(h, t, K)$ '. An analogous strengthening then also concerns 5.7 and, with $c$ and $\nu$ depending only on $n$, the results of Section 6 (excluding 6.3).

To achieve this, only minor modifications in the proofs are needed. In particular, let $f_{1}, \ldots, f_{s}$ be as in the proof of 4.9 , and choose $r>0$ such that the sets $Q_{i}^{\prime}=f_{i}^{-1} B^{m}(r)$ cover $Q^{m}$. Let $Y \in \mathcal{Y}_{n m}$ with $\operatorname{dim} Y \geq 2$. Then the open embeddings $f_{i} \times \mathrm{id} \mid:\left(Q_{i}^{\prime} \times \mathbf{R}^{n-m}\right) \cap Y^{*} \rightarrow Y$ for $1 \leq i \leq s$ form an atlas for $Y^{*}$ 
the coordinate changes of which are $\mathrm{BL}$ and, thus, $\mathrm{QC}$. In connection with $Y^{*}$ define $\mathrm{QC}$ embeddings referring to this atlas (cf. [Ku, I.2]).

\section{Main results in Euclidean spaces}

6.1. In this section we apply first the previously established statements $\mathcal{D}\left(\mathbf{R}^{n} ; \mathcal{Y}_{n}\right)$ and $\mathcal{D}\left(\mathbf{R}_{+}^{n} ; \mathcal{Y}_{n}^{+}\right)$to prove Theorem 6.2 , which generalizes both of them and also the result $\left[\mathrm{TV}_{2}, 3.4\right]$ of Sullivan's theory. We then use this theorem to prove Theorem 6.6, an analogue of it for the majorant topology.

6.2. Theorem. Let $n \geq 1$, let $(X, \mathcal{Y})$ denote either $\left(\mathbf{R}^{n}, \mathcal{Y}_{n}\right)$ or $\left(\mathbf{R}_{+}^{n}, \mathcal{Y}_{n}^{+}\right)$, let $U$ be an open subset of $X$, let $B, B^{\prime} \subset U$ be compact with $B \subset \subset B^{\prime}$ in $U$, and, for each $\gamma$ in a finite index set $\Gamma$, let $Y_{\gamma} \in \mathcal{Y}$ and let $C_{\gamma}$ and $C_{\gamma}^{\prime}$ be open subsets of $U \cap Y_{\gamma}$ with $C_{\gamma} \subset \subset C_{\gamma}^{\prime}$ in $U \cap Y_{\gamma}$. Let $\mathcal{Q}$ be a neighbourhood of id: $U \rightarrow X$ in $E(U ; X)$. Then there exist a neighbourhood $\mathcal{P}$ of id in $E(U ; X)$ and a continuous map $\varphi: \mathcal{P} \times I \rightarrow \mathcal{Q}$ such that if $h_{t}=\varphi(h, t)$ for $h \in \mathcal{P}, t \in I$, then the conditions (1)-(6) of 3.2 and the following ones are satisfied:

(7) If $\gamma \in \Gamma$, if $h \in \mathcal{P}$ respects $Y_{\gamma}$, and $h \mid C_{\gamma}^{\prime}=\mathrm{id}$, then $h_{t} \mid C_{\gamma}=\mathrm{id}$ for each $t \in I$.

(8) Let $\Lambda$ be a metric space, let $f=\left(f_{\lambda}\right)_{\lambda \in \Lambda} \in I_{\Lambda}(U ; X)$ with $f_{\lambda} \in \mathcal{P}$ for each $\lambda \in \Lambda$, and define $f^{*}=\varphi^{*}(f) \in I_{\Lambda \times I}(U ; X)$. Suppose that $\gamma \in \Gamma$, that $f$ respects $Y_{\gamma} \times \Lambda$, and that $f \mid C_{\gamma}^{\prime} \times \Lambda$ is (a) LIP or (b) locally $L$-BL or (c) $L-\mathrm{BL}$. Then $f^{*} \mid C_{\gamma} \times \Lambda \times I$ is, respectively, (a) LIP or (b) locally $L^{*}-\mathrm{BL}$ or (c) $L^{*}-\mathrm{BL}$ with $L^{*}$ depending only on $L$ and $n$.

(9) If $\gamma \in \Gamma$, if $h \in \mathcal{P}$ respects $Y_{\gamma}$, if $h \mid C_{\gamma}^{\prime}$ is (a) LQS or (b) locally $\eta-$ QS or (c) $\eta$-QS, and if $t \in I$, then $h_{t} \mid C_{\gamma}$ is, respectively, (a) LQS or (b) (if $\operatorname{dim} Y_{\gamma} \geq 2$ ) locally $\eta^{*}$-QS with $\eta^{*}$ depending only on $\eta$ and $n$ or (c) $\hat{\eta}-\mathrm{QS}$ with $\hat{\eta}$ depending only on $\eta$ and the sets given in the first sentence of the theorem.

Proof. Let $\kappa \in(0,1]$. This scaling number will be fixed later. Let $p$ denote an integer variable with $0 \leq p \leq n$. Let $T$ be the cell decomposition of $X$ consisting of all closed $p$-cubes for all $p$ with vertices in $\left(2 \kappa \mathbf{Z}^{n}\right) \cap X$ and with side length $2 \kappa$. Let $T_{p}=\{Q \in T \mid \operatorname{dim} Q=p\}$. For each $Q \in T_{p}$, choose an orthogonal map $S_{Q}: \mathbf{R}^{n} \rightarrow \mathbf{R}^{n}$ which permutes the standard basis of $\mathbf{R}^{n}$ such that if $a_{Q}$ is the centre of $Q$ and $\mu_{Q}(x)=\kappa S_{Q} x+a_{Q}$ for $x \in \mathbf{R}^{n}$, then $\mu_{Q} I^{p}=Q$. Note that if $Y \in \mathcal{Y}_{n}$, then $\mu_{Q} J^{n} \cap Y \neq \emptyset$ if and only if $Q \subset Y$. It follows that $\left\{J^{n} \cap \mu_{Q}^{-1} Y \mid Y \in \mathcal{Y}_{n}\right\} \subset\left\{J^{n} \cap Y \mid Y \in \mathcal{Y}_{n p} \cup\{\emptyset\}\right\}$. If $Q \subset \partial X$, we choose $S_{Q}$ in such a way that it and, hence, $\mu_{Q}$ respect $X$.

For all $p$, choose numbers $0<a_{p, n-p}<\cdots<a_{p, 0}<a_{p,-1}<a_{p}<1$ and $0<b_{p, n-p}<\cdots<b_{p, 0}<b_{p,-1}<b_{p}<1$ depending only on $n$ and $p$ such that $1-a_{p}>b_{p}$ and that $b_{p-1, n-p+1}>1-a_{p, n-p}$ if $p \geq 1$. For all $p, Q \in T_{p}$, and integers $j,-1 \leq j \leq n-p$, define sets $U_{p}=J^{p}\left(a_{p}\right) \times J^{n-p}\left(b_{p}\right), Q^{*}=\left(\mu_{Q} U_{p}\right) \cap X$, $B_{p, j}=I^{p}\left(a_{p, j}\right) \times I^{n-p}\left(b_{p, j}\right)$, and $Q^{j}=\left(\mu_{Q} B_{p, j}\right) \cap X$ in $\mathbf{R}^{n}$. Then

$$
\bar{Q}_{1}^{*} \cap \bar{Q}_{2}^{*}=\emptyset \quad \text { if } \quad Q_{1}, Q_{2} \in T, \quad Q_{1} \not \subset Q_{2} \text {, and } Q_{2} \not \subset Q_{1} \text {; }
$$




$$
\bigcup\left\{Q^{p-j} \mid Q \in T_{j}, \quad 0 \leq j \leq p\right\} \supset \bigcup T_{p} .
$$

Obviously, for all $p, i=0,1$, and $Q \in T_{p}$, the set $A_{p, i}^{\prime}=J^{p} \cap \mu_{Q}^{-1}\left[\bigcup\left\{Q_{1}^{p-j-i} \mid\right.\right.$ $\left.\left.Q_{1} \in T_{j}, \quad 0 \leq j<p\right\}\right]$ is independent of $Q$. Let $A_{p, i}=A_{p, i}^{\prime} \times J^{n-p}$. Then

$$
\mu_{Q}\left[A_{p, i} \cap U_{p}\right] \cap X=Q^{*} \cap\left(\bigcup\left\{Q_{1}^{p-j-i} \mid Q_{1} \in T_{j}, 0 \leq j<p\right\}\right) .
$$

For each $p$, let $\mathcal{Q}_{p}$ be the neighbourhood of id: $U_{p} \rightarrow J^{n}$ and $\psi_{p}$ the continuous map given by the statement $\mathcal{D}\left(J^{n} ; A_{p, 0}, A_{p, 1}, U_{p}, B_{p, 0}, B_{p,-1} ; \mathcal{Y}_{n} \mid J^{n}\right)$, valid by 5.5 and 3.11(i). Similarly, for each $p<n$, let $\mathcal{Q}_{p}^{+}$be the neighbourhood of id: $U_{p} \cap J_{+}^{n} \rightarrow J_{+}^{n}$ and $\psi_{p}^{+}$the continuous map given by the statement $\mathcal{D}\left(J_{+}^{n}\right.$; $\left.A_{p, 0} \cap J_{+}^{n}, A_{p, 1} \cap J_{+}^{n}, U_{p} \cap J_{+}^{n}, B_{p, 0} \cap J_{+}^{n}, B_{p,-1} \cap J_{+}^{n} ; \mathcal{Y}_{n}^{+} \mid J_{+}^{n}\right)$, valid by 5.6 and 3.11(i).

Looking more closely at the situation of the theorem, we choose for each $\gamma \in \Gamma$ a closed neighbourhood $C_{\gamma}^{*} \subset C_{\gamma}^{\prime}$ in $U \cap Y_{\gamma}$ of the closure $\widetilde{C}_{\gamma}$ of $C_{\gamma}$ in $U \cap Y_{\gamma}$. Define a finite subcomplex

$$
T^{0}=\left\{Q \in T \mid Q \subset Q_{1} \text { for some } Q_{1} \in T \text { with } Q_{1} \cap B \neq \emptyset\right\}
$$

of $T$ and its subcomplexes

$$
T^{\gamma}=\left\{Q \in T^{0} \mid Q \subset Q_{1} \text { for some } Q_{1} \in T^{0} \text { with } Q_{1} \cap C_{\gamma}^{*} \neq \emptyset\right\} \quad(\gamma \in \Gamma) .
$$

Then $Q^{*} \cap B=\emptyset$ if $Q \in T \backslash T^{0}$. We fix $\kappa$ so small that $Q^{*} \subset B^{\prime}$ if $Q \in T^{0}$, $\bar{Q}^{*} \cap Y_{\gamma} \subset C_{\gamma}^{\prime}$ if $\gamma \in \Gamma$ and $Q \in T^{\gamma}$, and $\bar{Q}^{*} \cap \widetilde{C}_{\gamma}=\emptyset$ if $\gamma \in \Gamma$ and $Q \in T^{0} \backslash T^{\gamma}$. Let $T_{p}^{0}=T^{0} \cap T_{p}$. For each $\gamma$, define open subsets $C_{\gamma}^{\prime}=C_{\gamma}^{-1} \supset C_{\gamma}^{0} \supset \cdots \supset C_{\gamma}^{n}$ of $U \cap Y_{\gamma}$ by $C_{\gamma}^{p}=C_{\gamma}^{p-1} \backslash \bigcup\left\{\bar{Q}^{*} \mid Q \in T_{p}^{0} \backslash T^{\gamma}\right\}$. Then $\widetilde{C}_{\gamma} \subset C_{\gamma}^{n}$, and $\bar{Q}^{*} \cap Y_{\gamma} \subset C_{\gamma}^{j}$ if $Q \in T^{\gamma},-1 \leq j \leq n$, and $\operatorname{dim} Q \geq j$. Set $B_{-1}=\emptyset$ and

$$
B_{p}=\bigcup\left\{Q^{p-j} \mid Q \in T_{j}^{0}, 0 \leq j \leq p\right\} \subset U \text { for each } p .
$$

Then $B_{n} \supset B, B_{p} \backslash B_{p-1} \subset \bigcup\left\{Q^{0} \mid Q \in T_{p}^{0}\right\}$, and, for each $Q \in T_{p}^{0}, \mu_{Q}\left[A_{p, 1} \cap\right.$ $\left.U_{p}\right] \cap X=Q^{*} \cap B_{p-1}$ and

$$
\mu_{Q}\left[\left(A_{p, 0} \cap U_{p}\right) \cup B_{p, 0}\right] \cap X=Q^{*} \cap B_{p} .
$$

Let $0 \leq p \leq n$. For each $Q \in T_{p}^{0}$, let $\left(U_{Q}, \mathcal{P}_{Q}, \varphi_{Q}\right)$ denote either $\left(U_{p}, \mathcal{Q}_{p}, \psi_{p}\right)$ whenever $Q \not \subset \partial X$ or $\left(U_{p} \cap J_{+}^{n}, \mathcal{Q}_{p}^{+}, \psi_{p}^{+}\right)$whenever $Q \subset \partial X$. Then $\mu_{Q} U_{Q}=Q^{*}$. There is a neighbourhood $\mathcal{P}_{p}$ of the inclusion map in $E\left(U, B_{p-1} ; X\right)$ such that for each $h \in \mathcal{P}_{p}$ and for each $Q \in T_{p}^{0}$, we have that $h Q^{*} \subset \mu_{Q} J^{n}$ and that $h_{Q}=\mu_{Q}^{-1} h \mu_{Q} \mid U_{Q} \in \mathcal{P}_{Q}$. We define a continuous map $\varphi_{p}: \mathcal{P}_{p} \times I \rightarrow E(U ; X)$ by setting

$$
\begin{gathered}
\varphi_{p}(h, t)\left|Q^{*}=\mu_{Q} \varphi_{Q}\left(h_{Q}, t\right) \mu_{Q}^{-1}\right| Q^{*} \quad \text { if } Q \in T_{p}^{0}, \\
\varphi_{p}(h, t)=h \quad \text { on } U \backslash \bigcup\left\{Q^{-1} \mid Q \in T_{p}^{0}\right\} .
\end{gathered}
$$


Then $\varphi_{p}$ satisfies the conditions (1), (2), and (4)-(6) of the theorem in place of $\varphi$, and $\varphi_{p}\left[\mathcal{P}_{p} \times 1\right] \subset E\left(U, B_{p} ; X\right)$. Thus, we may assume that $\varphi_{p}\left[\mathcal{P}_{p} \times 1\right] \subset \mathcal{P}_{p+1}$ if $p<n$ and that $\varphi_{p}\left[\mathcal{P}_{p} \times I\right] \subset \mathcal{Q}$ for each $p$. Hence, $\mathcal{P}=\mathcal{P}_{0}$ and $\varphi=$ $\varphi_{n} * \cdots * \varphi_{0}: \mathcal{P} \times I \rightarrow \mathcal{Q}$ satisfy the theorem except possibly for the conditions (7)-(9). We finally show that $\varphi$ also satisfies (7) and, if $\mathcal{P}_{0}, \ldots, \mathcal{P}_{n}$ are sufficiently small, (8) and (9). Here we assume that the composition of $\varphi_{0}, \ldots, \varphi_{n}$ is done in such a way that $\varphi(h, t)=\varphi_{p}(\varphi(h, p /(n+1)),(n+1) t-p)$ if $0 \leq p \leq n, h \in \mathcal{P}$, and $t \in I_{p}=[p /(n+1),(p+1) /(n+1)]$.

In proving (7)-(9), we may assume that $\Gamma$ consists of only one element, $\gamma$, say. We first prove for each $p$ the conditions $\left(7_{p}\right),\left(8_{p}\right)$, and $\left(9_{p}\right)$ which we obtain from the conditions (7), (8), and (9), respectively, of the theorem replacing $\left(\mathcal{P}, \varphi, C_{\gamma}, C_{\gamma}^{\prime}\right)$ by $\left(\mathcal{P}_{p}, \varphi_{p}, C_{\gamma}^{p}, C_{\gamma}^{p}\right)$. Let $\mathcal{P}_{p}^{\gamma}=\left\{h \in \mathcal{P}_{p} \mid h\right.$ respects $\left.Y_{\gamma}\right\}$ and $\mathcal{P}^{\gamma}=\mathcal{P}_{0}^{\gamma}$. Let $T_{p}^{\gamma}=T^{\gamma} \cap T_{p}^{0}$. Recall that $\bar{Q}^{*} \cap Y_{\gamma} \subset C_{\gamma}^{p}$ if $Q \in T_{p}^{\gamma}$, and note that $Q^{*} \cap C_{\gamma}^{p}=\emptyset$ if $Q \in T_{p}^{0} \backslash T_{p}^{\gamma}$. It follows that if $(h, t) \in \mathcal{P}_{p}^{\gamma} \times I$, then $\varphi_{p}(h, t)$ defines a homeomorphism $C_{\gamma}^{p} \rightarrow h C_{\gamma}^{p}$, which is the identity whenever $h \mid C_{\gamma}^{p}=$ id. Thus, $\left(7_{p}\right)$ holds. This implies that (7) also holds. Now, if $Q \in T_{p}^{\gamma}$, let $Y_{\gamma Q}$ denote the element of $\mathcal{Y}_{n p} \cup\{\emptyset\}$ for which $\mu_{Q} J^{n} \cap Y_{\gamma}=\mu_{Q}\left[J^{n} \cap Y_{\gamma Q}\right]$ (then $Y_{\gamma Q} \subset \mathbf{R}_{+}^{n}$ whenever $Q \subset \partial X)$. Furthermore, let $U_{\gamma Q}=U_{Q} \cap Y_{\gamma Q}$.

To prove $\left(8_{p}\right)$, let $\Lambda$ be a metric space, and let $f=\left(f_{\lambda}\right)_{\lambda \in \Lambda} \in I_{\Lambda}(U ; X)$ be such that $f_{\lambda} \in \mathcal{P}_{p}^{\gamma}$ for each $\lambda \in \Lambda$ and that $f \mid C_{\gamma}^{p} \times \Lambda$ is (a) LIP or (b) locally $L-\mathrm{BL}$ or (c) $L-\mathrm{BL}$. Consider $f^{(p)}=\varphi_{p}^{*}(f) \in I_{\Lambda \times I}(U ; X)$. Let $Q \in T_{p}^{\gamma}$. Let $\Lambda_{\kappa}$ denote $\Lambda$ with the metric of $\Lambda$ divided by $\kappa$. Then the homeomorphism $\left(J^{n} \cap Y_{\gamma Q}\right) \times \Lambda_{\kappa} \rightarrow\left(\mu_{Q} J^{n} \cap Y_{\gamma}\right) \times \Lambda$ defined by $\mu_{Q} \times$ id multiplies the distances by $\kappa$. It follows that $\left(f_{\lambda Q} \mid U_{\gamma Q}\right)_{\lambda \in \Lambda_{\kappa}} \in I_{\Lambda_{\kappa}}\left(U_{\gamma Q} ; Y_{\gamma Q}\right)$ is (a) LIP or (b) locally $L$-BL or (c) $L-\mathrm{BL}$, respectively. Hence, $\left(\varphi_{Q}\left(f_{\lambda Q}, t\right) \mid U_{\gamma Q}\right)_{\lambda \in \Lambda_{\kappa}, t \in I} \in I_{\Lambda_{\kappa} \times I}\left(U_{\gamma Q} ; Y_{\gamma Q}\right)$ is (a) LIP or (b) locally $L_{1}-\mathrm{BL}$ or (c) $L_{1}-\mathrm{BL}$, respectively, with $L_{1} \geq L$ depending only on $L$ and $n$. A direct estimation shows that then $f^{(p)} \mid\left(Q^{*} \cap Y_{\gamma}\right) \times \Lambda \times I$ is, respectively, (a) LIP or (b) locally $L_{1}-\mathrm{BL}$ or (c) $L_{1}-\mathrm{BL}$. It follows that $f^{(p)} \mid C_{\gamma}^{p} \times \Lambda \times I$ is LIP in $\left(8_{p} \mathrm{a}\right)$ and locally $L_{1}-\mathrm{BL}$ in $\left(8_{p} \mathrm{~b}\right)$. In $\left(8_{p} \mathrm{c}\right)$, for $\mathcal{P}_{p}$ small enough, it follows from 2.4 that $f^{(p)} \mid\left(\bigcup\left\{Q^{*} \cap Y_{\gamma} \mid Q \in T_{p}^{\gamma}\right\}\right) \times \Lambda \times I$ is $2 L_{1}-\mathrm{BL}$ and then from 2.7 that $f^{(p)} \mid C_{\gamma}^{p} \times \Lambda \times I$ is $4 L_{1}-\mathrm{BL}$.

To prove (8), choose an open subset $C_{\gamma}^{\prime \prime}$ of $Y_{\gamma}$ with $C_{\gamma} \subset \subset C_{\gamma}^{\prime \prime} \subset \subset C_{\gamma}^{n}$ in $U \cap Y_{\gamma}$. We can choose $\mathcal{P}$ so small that for each $(h, t) \in \mathcal{P}^{\gamma} \times I$,

$$
h_{t}\left[B^{\prime} \cap C_{\gamma}\right] \cap h\left[\left(B^{\prime} \cap Y_{\gamma}\right) \backslash C_{\gamma}^{\prime \prime}\right]=\emptyset, \quad h\left[B^{\prime} \cap C_{\gamma}^{\prime \prime}\right] \cap h_{t}\left[\left(B^{\prime} \cap Y_{\gamma}\right) \backslash C_{\gamma}^{n}\right]=\emptyset,
$$

implying, as $h_{t}\left[B^{\prime} \cap Y_{\gamma}\right]=h\left[B^{\prime} \cap Y_{\gamma}\right]$, that $h_{t}\left[B^{\prime} \cap C_{\gamma}\right] \subset h\left[B^{\prime} \cap C_{\gamma}^{\prime \prime}\right] \subset h_{t}\left[B^{\prime} \cap C_{\gamma}^{n}\right]$ and, thus, that $h_{t} C_{\gamma} \subset h C_{\gamma}^{\prime \prime} \subset h_{t} C_{\gamma}^{n}$. Now suppose that $\Lambda, f, f^{*}, \gamma, L$ are as in (8). Then the conditions $\left(8_{0} i\right), \ldots,\left(8_{n} i\right)$ for $i$ equal to $\mathrm{a}, \mathrm{b}$, or $\mathrm{c}$ imply that $f^{*} \mid C_{\gamma}^{p} \times \Lambda \times I_{p}$ is for each $p$, respectively, (a) LIP or (b) locally $L^{\prime}-\mathrm{BL}$ or (c) $L^{\prime}-\mathrm{BL}$ with $L^{\prime}$ depending only on $L$ and $n$. Consider (8c). Since $(x, \lambda, t) \in f^{*}\left[C_{\gamma} \times \Lambda \times I\right]$ implies that $(x, \lambda, s) \in f^{*}\left[C_{\gamma}^{n} \times \Lambda \times I\right]$ for each $s \in I$, the proof of 2.10 shows 
that $f^{*} \mid C_{\gamma} \times \Lambda \times I$ is $2 L^{\prime}-\mathrm{BL}$. The conditions (8a) and (8b), with $L^{*}=2 L^{\prime}$, are verified similarly but more easily.

To prove $\left(9_{p} \mathrm{c}\right)$, suppose that $h \in \mathcal{P}_{p}^{\gamma}$ is $\eta-\mathrm{QS}$ on $C_{\gamma}^{p}$. Let $Q \in T_{p}^{\gamma}$. Since $\mu_{Q}$ is a similarity, $h_{Q} \mid U_{\gamma Q}$ is $\eta$-QS. Hence, $\varphi_{Q}\left(h_{Q}, t\right) \mid U_{\gamma Q}$ for $t \in I$ is $\eta_{1}-Q S$ with $\eta_{1}$ depending only on $\eta$ and $n$. Thus, also $\varphi_{p}(h, t) \mid Q^{*} \cap Y_{\gamma}$ is $\eta_{1}-\mathrm{QS}$. It follows, by 2.5 and 2.9 , that if $\mathcal{P}_{p}$ is small enough, $\varphi_{p}(h, t) \mid C_{\gamma}^{p}$ for $t \in I$ is $\eta_{2}-\mathrm{QS}$ with $\eta_{2}$ not depending on $(h, t)$.

The proofs of $\left(9_{p} \mathrm{a}\right)$ and $\left(9_{p} \mathrm{~b}\right)$ are similar but simpler. The condition (9) follows. ㅁ

6.3. Remark. To 6.2 , we can add the modifications of the conditions (5)-(9) in which $Y$ and $Y_{\gamma}$ are of the same form $Y_{1} \cup Y_{2}$ as in 5.8 and in which $h, h^{\prime}$, and $f_{\lambda}$ for $\lambda \in \Lambda$ respect $Y_{1}$ and $Y_{2}$. For (8) and (9) this is shown by an application of 5.8 to the proof of 6.2 . We can similarly strengthen $6.4,6.6$, and 6.7 below.

The following result generalizes $\mathcal{D}^{*}\left(\mathbf{R}^{n} ; \mathcal{Y}_{n}\right)$ and $\mathcal{D}^{*}\left(\mathbf{R}_{+}^{n} ; \mathcal{Y}_{n}^{+}\right)$.

6.4. Corollary. The modification of 6.2 holds where $\mathcal{Q}$ is a neighbourhood of id in $H(X)$, where the conditions (2)-(4) and (6) of 3.2 are replaced by the respective conditions of 3.6 , and where in (8) and (9) the set $C_{\gamma}$ is replaced by $C_{\gamma} \cup\left(Y_{\gamma} \backslash B^{\prime}\right)$.

Proof. Let $B_{1}$ and $B_{1}^{\prime}$ be compact sets with $B \subset \subset B_{1} \subset \subset B_{1}^{\prime} \subset \subset B^{\prime}$. For each $\gamma$, let $C_{\gamma}^{\prime \prime}$ be an open subset of $Y_{\gamma}$ with $C_{\gamma} \subset \subset C_{\gamma}^{\prime \prime} \subset \subset C_{\gamma}^{\prime}$ in $U \cap Y_{\gamma}$. Apply 6.2 replacing $\left(B, B^{\prime},\left(C_{\gamma}\right)_{\gamma \in \Gamma}\right)$ by $\left(B_{1}, B_{1}^{\prime},\left(C_{\gamma}^{\prime \prime}\right)_{\gamma \in \Gamma}\right)$ and substituting $E(U ; X)$ for $\mathcal{Q}$. Let $\mathcal{P}$ be the neighbourhood and $\varphi_{0}$ the map thus obtained. We may assume that $h B \subset B_{1}$ if $h \in \mathcal{P}$ and (cf. the proof of 6.2(8)) that $h C_{\gamma} \subset \varphi_{0}(h, t) C_{\gamma}^{\prime \prime}$ for each $h \in \mathcal{P}$ respecting $Y_{\gamma}$. Define $\varphi(h, t)$ extending $\varphi_{0}(h, t)^{-1} h$ by id. If $\mathcal{P}$ is small enough, $\varphi$ satisfies ( $8 \mathrm{c}$ ) and (9c) by 2.7 or 2.9 , respectively. $\square$

6.5. Majorant topology. The next theorem is a modification of 6.2 to the case where $B$ is allowed to be a possibly noncompact closed subset of $U$. We now must consider only embeddings $h \in E(U ; X)$ that are sufficiently close to the inclusion map in the majorant topology. It can be shown by the aid of $[\mathrm{Si}, 1.7]$ that if $X$ is a locally compact, locally connected metric space and $U \subset X$ is open, there is a majorant neighbourhood $\mathcal{P}$ of id in $E(U ; X)$ such that $h U=U$ for each $h \in \mathcal{P}$, i.e., $\mathcal{P} \subset H(U)$. Thus, without loss of generality, it suffices to only consider self-homeomorphisms of $U$.

Let $X$ be a metric space. We will use the fact [KS, pp. 46-47] that relative to the majorant topology, $H(X)$ is a topological group. For the majorant topology of $I(X)$, note that for each $\varepsilon \in C(X \times I ;(0, \infty))$ there is $\varepsilon^{\prime} \in C(X ;(0, \infty))$ with $\varepsilon^{\prime}(x) \leq \varepsilon(x, t)[\mathrm{KS}$, Lemma on p. 47].

6.6. Theorem. Let $n, X, \mathcal{Y}, U, B, B^{\prime}$, and $\left(Y_{\gamma}, C_{\gamma}, C_{\gamma}^{\prime}\right)_{\gamma \in \Gamma}$ be otherwise the same as in 6.2, but, more generally, suppose that $B$ and $B^{\prime}$ are only closed 
in $U$, not necessarily compact, and allow $\Gamma$ to be possibly infinite still supposing that the family $\left(C_{\gamma}\right)_{\gamma \in \Gamma}$ is locally finite in $U$. Let $\mathcal{Q}$ be a neighbourhood of id in $H(U)$ with respect to the majorant topology. Then there exist a neighbourhood $\mathcal{P}$ of id in $H(U)$ with respect to the majorant topology and a continuous map $\varphi: \mathcal{P} \times I \rightarrow \mathcal{Q}$ (that is, in the compact-open topologies of $\mathcal{P}$ and $\mathcal{Q}$ ) satisfying the conditions (1)-(9) in 6.2, with (9c) excluded, such that the induced continuous map $\varphi^{*}: \mathcal{P} \rightarrow I(U)$ is also continuous in the majorant topologies of $\mathcal{P}$ and $I(U)$.

Proof. We may assume that $\mathcal{Q}=H(U) \cap N_{\varepsilon}$ (id) for some $\varepsilon \in C(U ;(0, \infty))$.

We first prove the special case where $B$ is the union of a disjoint family $\left(B_{j}\right)_{j \geq 1}$ of compact sets each open in $B$. Choose for each $j$ an open neighbourhood $U_{j}$ of $B_{j}$ in $U$ with $\bar{U}_{j} \subset B^{\prime}$ compact such that the family $\left(\bar{U}_{j}\right)_{j \geq 1}$ is disjoint and locally finite in $U$. Then for each $j$ choose a compact neighbourhood $B_{j}^{\prime}$ of $B_{j}$ in $U_{j}$ and a positive number $\varepsilon_{j}$ with $\varepsilon_{j} \leq \min \varepsilon \bar{U}_{j}$. For each $j$, we apply 6.2 with $U_{j}, B_{j}, B_{j}^{\prime}, \Gamma_{j}=\left\{\gamma \in \Gamma \mid C_{\gamma} \cap U_{j} \neq \emptyset\right\}, C_{\gamma} \cap U_{j}, C_{\gamma}^{\prime} \cap U_{j}, E\left(U_{j} ; X\right)$ substituted, respectively, for $U, B, B^{\prime}, \Gamma, C_{\gamma}, C_{\gamma}^{\prime}, \mathcal{Q}$; let $\mathcal{P}_{j}$ be the set and $\varphi_{j}$ the map we thus get. Then there is a number $\delta_{j}>0$ such that if $\mathcal{P}_{j}^{\prime}=E\left(U_{j} ; X\right) \cap N_{\delta_{j}}(\mathrm{id})$, then $\mathcal{P}_{j}^{\prime} \subset \mathcal{P}_{j}$ and $\varphi_{j}\left[\mathcal{P}_{j}^{\prime} \times I\right] \subset N_{\varepsilon_{j}}(\mathrm{id})$. Choose $\delta \in C(U ;(0, \infty))$ such that $\delta \leq \varepsilon$ and $\delta(x) \leq \delta_{j}$ for $x \in U_{j}, j \geq 1$. Let $\mathcal{P}=H(U) \cap N_{\delta}($ id $)$. For $h \in \mathcal{P}, t \in I$, define $\varphi(h, t)=h_{t}$ in $\mathcal{Q}$ by

$$
h_{t}(x)= \begin{cases}\varphi_{j}\left(h \mid U_{j}, t\right)(x) & \text { if } x \in U_{j}, j \geq 1, \\ h(x) & \text { if } x \in U \backslash \bigcup_{j \geq 1} B_{j}^{\prime} .\end{cases}
$$

Then $\varphi$ is the desired map if only for (8c) we choose the $\varepsilon_{j}$ 's and $\delta$ more carefully so as to satisfy, in addition, $3 \varepsilon_{j} \leq d\left(\bar{U}_{j}, \bar{U}_{k}\right)$ if $j \neq k, 3 \varepsilon_{j} \leq d\left(B_{j}^{\prime}, U \backslash U_{j}\right)$ if $j \geq 1$, and $3 \delta(x) \leq d\left(x, \bigcup_{j \geq 1} B_{j}^{\prime}\right)$ if $x \in U \backslash \bigcup_{j \geq 1} U_{j}$. Namely, these inequalities yield the necessary estimates for $d\left(\varphi^{*}(f)(x, \lambda, t), \varphi^{*}(f)\left(x^{\prime}, \lambda^{\prime}, t^{\prime}\right)\right)$ if $x \in B_{j}^{\prime}$ and either $x^{\prime} \in U_{k}(k \neq j)$ or $x^{\prime} \notin \bigcup_{k \geq 1} U_{k}$.

We now prove the general case. Choose a closed subset $B^{\prime \prime}$ of $U$ with $B \subset \subset$ $B^{\prime \prime} \subset \subset B^{\prime}$ in $U$. For each $\gamma \in \Gamma$ choose open subsets $C_{\gamma}^{\prime \prime}$ and $C_{\gamma}^{3}$ of $Y_{\gamma}\left(C_{\gamma}^{3}\right.$ is needed only for (8c)) such that $C_{\gamma} \subset \subset C_{\gamma}^{3} \subset \subset C_{\gamma}^{\prime \prime} \subset \subset C_{\gamma}^{\prime}$ in $U \cap Y_{\gamma}$ and such that $\left(C_{\gamma}^{\prime \prime}\right)_{\gamma \in \Gamma}$ is locally finite in $U$. Using [Si, 1.7] it is easy to see that assuming $\varepsilon$ to be small enough, we have for each $\gamma \in \Gamma$ that $h C_{\gamma} \subset h^{\prime} C_{\gamma}^{3}$ whenever $h, h^{\prime} \in \mathcal{Q}$ respect $Y_{\gamma}$. In a standard way, choose a family $\left(D_{j}\right)_{j \geq 1}$ of compact subsets of $U$ whose interiors (in $U$ ) cover $U$ and for which $D_{i} \cap D_{j}=\emptyset$ if $|i-j|>1$. Then $E_{1}=\bigcup_{i \geq 1} D_{2 i-1}$ and $E_{2}=\bigcup_{i>1} D_{2 i}$ are closed in $U$, and $U=\operatorname{int} E_{1} \cup \operatorname{int} E_{2}$. Let $B_{k}=B \cap E_{k}$ and $B_{k}^{\prime \prime}=B^{\prime \prime} \cap E_{k}, k=1,2$. There is an open neighbourhood $C$ of $B \backslash \operatorname{int} E_{2}$ in $U$ with $C \subset \subset C^{\prime}=\operatorname{int} B_{1}^{\prime \prime}$ in $U$. We can then apply the special case above substituting $B_{2}$ for $B$ and the family $\left(Y_{\gamma}, C_{\gamma}^{3}, C_{\gamma}^{\prime \prime}\right)_{\gamma \in \Gamma}$, extended by $\left(X, C, C^{\prime}\right)$, for $\left(Y_{\gamma}, C_{\gamma}, C_{\gamma}^{\prime}\right)_{\gamma \in \Gamma}$. Let $\mathcal{P}_{2} \subset \mathcal{Q}$ be the set and $\varphi_{2}: \mathcal{P}_{2} \times I \rightarrow \mathcal{Q}$ the map thus obtained. The sets $C$ and $C^{\prime}$ will only be needed in (7); note that $B \subset B_{2} \cup C$. We can now again apply the special case, with $B_{1}^{\prime \prime}$ substituted for 
$B, C_{\gamma}^{\prime \prime}$ for $C_{\gamma}(\gamma \in \Gamma)$, and $\mathcal{P}_{2}$ for $\mathcal{Q}$. Let $\mathcal{P}$ be the set and $\varphi_{1}: \mathcal{P} \times I \rightarrow \mathcal{P}_{2}$ the map thus obtained. Then $\varphi=\varphi_{2} * \varphi_{1}: \mathcal{P} \times I \rightarrow \mathcal{Q}$, defined as in 3.10 , is the desired map. $\square$

6.7. Corollary. The modification of 6.6 holds where the conditions (2)-(4) are replaced, respectively, by the conditions $h_{0}=\mathrm{id}, h_{1}|B=h| B$, and $h_{t} \mid U \backslash B^{\prime}=$ id.

Proof. By (1), we may assume that $\mathcal{Q}=H(U)$. Apply 6.6 for $\mathcal{Q}=H(U)$ with $B$ and $\left(C_{\gamma}\right)_{\gamma \in \Gamma}$ replaced by a set $B^{\prime \prime}$ and a family $\left(C_{\gamma}^{\prime \prime}\right)_{\gamma \in \Gamma}$ as defined in the proof of 6.6. Let $\mathcal{P}$ be the set and $\varphi_{0}$ the map thus given. We may assume that $h B \subset B^{\prime \prime}$ if $h \in \mathcal{P}$ and that $h C_{\gamma} \subset \varphi_{0}(h, t) C_{\gamma}^{\prime \prime}$ if $h \in \mathcal{P}$ respects $Y_{\gamma}$. Set $\varphi(h, t)=\varphi_{0}(h, t)^{-1} h$ for $h \in \mathcal{P}, t \in I$. $\square$

\section{Deformation on manifolds}

7.1. Terminology. Let $0 \leq p \leq n$ be integers, and let CAT denote either LIP or LQS. We call a separable metric space $M$ a CAT $n$-manifold if each point of $M$ has an open neighbourhood CAT homeomorphic to an open subset of $\mathbf{R}_{+}^{n}$. (A definition based on atlases is essentially equivalent; see [LT, 4.7].) A subset of a CAT manifold $M$ is called a CAT submanifold of $M$ if it is a CAT manifold in the induced metric. Such is the boundary $\partial M$ of $M$.

Define $Y_{n, 0}=\{0\} \subset \mathbf{R}^{n}, Y_{n, 1}=\left\{x \in \mathbf{R}^{n} \mid x_{1}=\cdots=x_{n-1}=0, x_{n} \geq 0\right\}$ if $n \geq 1$, and $Y_{n, p}=\left\{x \in \mathbf{R}^{n} \mid x_{1}=\cdots=x_{n-p}=0, x_{n-1} \geq 0, x_{n} \geq 0\right\}$ if $2 \leq p \leq n$. We call a subset $N$ of a CAT $n$-manifold $M$ a locally CAT flat $p$-submanifold of $M$ if for each point $x \in N$ there exist an open neighbourhood $V$ of $x$ in $M$, an open subset $W$ of $\mathbf{R}^{n}$ or of $\mathbf{R}_{+}^{n}$ whenever $p \leq 1$ or of $\mathbf{R}_{+}^{n}$ whenever $p \geq 2$, and a CAT homeomorphism $f: V \rightarrow W$ such that $f[V \cap N]=W \cap Y_{n, p}$. This means, in particular, that $N$ is a CAT submanifold of $M$, which meets $\partial M$ transversally in a certain sense.

If $N$ is a locally CAT flat $p$-submanifold of $\partial M$, for each point $x \in N$ there exist an open neighbourhood $V$ of $x$ in $M$ and an open CAT embedding $f: V \rightarrow \mathbf{R}_{+}^{n}$ such that $V \cap N=f^{-1} \mathbf{R}_{+}^{p}\left(\right.$ see $\left[\mathrm{TV}_{3}, 3.12\right]$ for $\left.\mathrm{CAT}=\mathrm{LQS}\right)$.

7.2. Setting. In this section we consider deformation of embeddings on manifolds in the following three cases. In Case 1 and in Case 2, we let CAT be, respectively, LIP or LQS, let $M$ be a CAT $n$-manifold ( $n \geq 0)$, let $N$ be a closed locally CAT flat $p$-submanifold (possibly empty) of $M(0 \leq p \leq n)$ or of $\partial M$ $(0 \leq p<n)$, and let $\mathcal{Y}=\{M, \partial M, N, \partial N, N \cap \partial M, \operatorname{cl}(\partial N \backslash \partial M), \partial(N \cap \partial M)$, $\operatorname{cl}(M \backslash N), \partial \operatorname{cl}(M \backslash N), \operatorname{cl}(\partial M \backslash N)\}$. In Case 3, we let CAT $=$ LIP, let $N$ be a LIP $(n-1)$-manifold $(n \geq 1)$, let $M$ be the product LIP $n$-manifold $N \times I$, and let $\mathcal{Y}=\{M, \partial M, N \times 0, N \times 1, \partial N \times I, \partial N \times 0, \partial N \times 1\}$. Note that in all three cases the elements of $\mathcal{Y}$ are closed CAT submanifolds of $M$. In Cases 1 and 2 , note further that if $N \not \subset \partial M, p \geq 1$, and $h$ is an open embedding of an open 
subset of $M$ into $M$ which respects $\partial N$, then $h$ respects $N \cap \partial M=\partial N \cap \partial M$ and $\operatorname{cl}(\partial N \backslash \partial M)$, too.

In the proofs of $7.3-7.6$ we assume that $n \geq 1$; these results are trivially true if $n=0$.

7.3. Theorem. In Cases 1 and $3, \mathcal{D}(M ; \mathcal{Y})$ and $\mathcal{D}^{*}(M ; \mathcal{Y})$ hold. In Case 2 , $\mathcal{D}_{Q S}(M ; \mathcal{Y})$ and $\mathcal{D}_{Q S}^{*}(M ; \mathcal{Y})$ hold.

Proof. In Cases 1 and $3, \mathcal{D}(M ; \mathcal{Y})$ immediately follows from 5.5, 5.6, 5.8, 3.13 (cf. the proof of 7.4 ), and 3.12. By $3.9, \mathcal{D}^{*}(M ; \mathcal{Y}$ ) follows. The theorem for Case 2 is proved similarly (recall 3.7 .1 ). $\square$

We devote the rest of this section to generalizations of 7.3.

7.4. Theorem. In Cases 1 and 3, the analogues of 6.2 and 6.4 hold where $(X, \mathcal{Y})$ is $(M, \mathcal{Y})$, where $L^{*}$ in (8) depends only on $L$ and the family $\tau$ of all the displayed subsets of $M$, and where $\eta^{*}$ and $\hat{\eta}$ in (9) depend only on $\eta$ and $\tau$.

Proof. We first deduce from 6.2 its analogue in question-let it be called Theorem A-assuming, without loss of generality, that $\mathcal{Q}=E(U ; M)$. We choose open subsets $V_{1}, \ldots, V_{r}$ of $U$ in $B^{\prime}$ such that for each $V_{i}$ there is a LIP homeomorphism $g_{i}$ of $V_{i}$ onto $\mathbf{R}^{n}$ or onto $\mathbf{R}_{+}^{n}$ having the following properties: The sets $A_{i}=g_{i}^{-1} \bar{B}^{n}$ cover $B$, and for each $Y \in \mathcal{Y}$, either $g_{i}\left[V_{i} \cap Y\right] \in \mathcal{Y}_{n} \cup\{\emptyset\}$ or, in Case 1 with $p \geq 2$, with $N \cap \partial V_{i} \neq \emptyset \neq \partial N \cap \operatorname{Int} V_{i}$, and with $Y=\partial N$ or (if $p=n) Y=\partial \operatorname{cl}(M \backslash N)$, the set $g_{i}\left[V_{i} \cap Y\right]$ is the union of two half-spaces in $\mathcal{Y}_{n}$ meeting at their common boundary. Let $M_{i}=g_{i}^{-1} B^{n}(5), U_{i}=g_{i}^{-1} B^{n}(4)$, $B_{i}^{\prime}=g_{i}^{-1} \bar{B}^{n}(3)$, and $D_{i}^{\prime \prime}=g_{i}^{-1} B^{n}(2)$. Let $A_{i}^{*}=A_{1} \cup \cdots \cup A_{i}$ if $0 \leq i \leq r$. Define inductively an open neighbourhood $D_{i}$ of $A_{i}^{*}$ in $U$ as follows. Let $D_{0}=\emptyset$. For $i>0$, choose an open neighbourhood $D_{i}^{\prime} \subset \subset D_{i-1}$ of $A_{i-1}^{*}$ in $U$ and set $D_{i}=D_{i}^{\prime} \cup D_{i}^{\prime \prime}$. Finally, if $\gamma \in \Gamma$, choose open subsets $C_{\gamma}^{0}, C_{\gamma}^{1}, \ldots, C_{\gamma}^{r+1}$ of $U \cap Y_{\gamma}$ such that $C_{\gamma}^{0}=C_{\gamma}^{\prime}, C_{\gamma}^{r+1}=C_{\gamma}$, and $C_{\gamma}^{i} \subset \subset C_{\gamma}^{i-1}$ in $U \cap Y_{\gamma}$ for $i \geq 1$.

Let $1 \leq i \leq r$. As $g_{i} \mid M_{i}$ is BL, 6.2 and 6.3 imply that Theorem $A$ holds if we substitute $\left(M_{i}, \mathcal{Y} \mid M_{i}, U_{i}, \bar{D}_{i}^{\prime \prime}, B_{i}^{\prime}\right)$ for $\left(M, \mathcal{Y}, U, B, B^{\prime}\right)$ and $\left(Y_{\gamma} \cap M_{i}, C_{\gamma}^{i} \cap U_{i}\right.$, $\left.C_{\gamma}^{i-1} \cap U_{i}\right)_{\gamma \in \Gamma}$, extended by $\left(M_{i}, D_{i}^{\prime} \cap U_{i}, D_{i-1} \cap U_{i}\right)$, for $\left(Y_{\gamma}, C_{\gamma}, C_{\gamma}^{\prime}\right)_{\gamma \in \Gamma}$. Let $\mathcal{P}_{i}^{\prime}$ be the set and $\varphi_{i}^{\prime}$ the map thus obtained. Then there is a neighbourhood $\mathcal{P}_{i}$ of id in $E(U ; M)$ such that $h \in \mathcal{P}_{i}$ implies $h U_{i} \subset M_{i}$ and $h \mid U_{i} \in \mathcal{P}_{i}^{\prime}$. For $(h, t) \in \mathcal{P}_{i} \times I$, extend $\varphi_{i}^{\prime}\left(h \mid U_{i}, t\right)$ by $h \mid U \backslash U_{i}$ to $\varphi_{i}(h, t) \in E(U ; M)$. Then $\varphi_{i}: \mathcal{P}_{i} \times I \rightarrow E(U ; M)$ satisfies Theorem A in place of $\varphi$, with the possible exception of (8c) and (9c), if $B, C_{\gamma}, C_{\gamma}^{\prime}(\gamma \in \Gamma)$ are replaced by $\bar{D}_{i}^{\prime \prime}, C_{\gamma}^{i}, C_{\gamma}^{i-1}(\gamma \in \Gamma)$. Moreover, if $h \in \mathcal{P}_{i}$ and $h \mid D_{i-1}=\mathrm{id}$, then $\varphi_{i}(h, 1) \mid D_{i}=\mathrm{id}$. If $\mathcal{P}_{i}$ is sufficiently small, (8c) and (9c) follow from 2.7 or 2.9 , respectively.

We may assume that $\varphi_{i}\left[\mathcal{P}_{i} \times 1\right] \subset \mathcal{P}_{i+1}$ for $i<r$. As $D_{r} \supset B$, it follows that $\mathcal{P}=\mathcal{P}_{1}$ and $\varphi=\varphi_{r} * \cdots * \varphi_{1}$ satisfy Theorem A, at least if for (8c) we choose $\mathcal{P}$ so small that $h_{t} C_{\gamma} \subset h_{t^{\prime}} C_{\gamma}^{r}$ for all $t, t^{\prime} \in I$ whenever $h \in \mathcal{P}$ respects $Y_{\gamma} ;$ cf. the proof of $(8 \mathrm{c})$ in 6.2 . 
The analogue of 6.4 in question is deduced from Theorem A as 6.4 is deduced from 6.2. ㅁ

7.5. Theorem. In Cases 1 and 3, the analogues of 6.6 and 6.7 hold where $(X, \mathcal{Y})$ is $(M, \mathcal{Y})$ and where the parts $(8 \mathrm{a})$ and $(9 \mathrm{a})$ only are retained of the conditions (8) and (9) stated in 6.2.

Proof. The analogue of 6.6 is deduced from 7.4 as 6.6 is deduced from 6.2, and it implies the analogue of 6.7 as 6.6 implies 6.7. $\square$

7.6. Theorem. In Case 2, the analogues of 7.4 and 7.5 hold where the condition (8) is deleted.

This is proved as 7.4 and 7.5.

7.7. Remark. For CAT $=$ TOP, i.e., for topological manifolds, 7.4 and 7.5 with (8) and (9) deleted are proved similarly. These results are due to Chernavskir [Ch, 1.22 and 5.1] and Edwards-Kirby [EK, 5.1 and Note after it, 6.2 and Remark after it, 7.2].

\section{Applications}

8.1. Local contractibility. By [Ch, 1.14] and [EK, 1.1], the homeomorphism group $H(M)$ of a compact topological manifold $M$ is locally contractible. If $(M, \mathcal{Y})$ is as in Case 1 or in Case 3 of 7.2 and if $M$ is compact, the statement $\mathcal{D}(M ; \emptyset, \emptyset, M, M, M ; \mathcal{Y})$, valid by 7.3 , implies that along with the group $H(M)$, certain of its subgroups are locally contractible (cf. [EK, 7.3]). If $(M, \mathcal{Y})$ is as in Case 2 of 7.2 and $M$ is compact, a similar fact follows from $\mathcal{D}_{Q S}(M ; \mathcal{Y})$. It is well known ([Ch, 1.18], [EK, p. 77]) that if $M$ is a noncompact manifold, $H(M)$ need not be locally contractible. However, if we consider $H(M)$ and $I(M)$ with the majorant topology, a weak form of local contractibility holds even then ([Ch, 1.13], [EK, 6.2]). In our case this is shown by 6.6 and by its analogues in 7.5 and 7.6 for the group $H(U)$ considered and for some of its subgroups if we substitute $U$ for $B$ and for $B^{\prime}$ and $(Y, U \cap Y, U \cap Y)_{Y \in \mathcal{Y}}$ for $\left(Y_{\gamma}, C_{\gamma}, C_{\gamma}^{\prime}\right)_{\gamma \in \Gamma}$.

Chernavski1 [Ch, 1.21] showed that $H(M)$ is locally contractible also whenever $M$ is homeomorphic to the interior of a compact manifold. We prove two results in this direction, Theorems 8.2 and 8.4 , the first of which implies that along with $H\left(\mathbf{R}^{n}\right)$, certain of its subgroups are locally contractible.

8.2. Theorem. Let $n \geq 1$, and let $(X, \mathcal{Y})$ denote either $\left(\mathbf{R}^{n}, \mathcal{Y}_{n}\right)$ or $\left(\mathbf{R}_{+}^{n}, \mathcal{Y}_{n}^{+}\right)$. Then there exist a neighbourhood $\mathcal{P}$ of id in $H(X)$ and a continuous map $\varphi: \mathcal{P} \times I \rightarrow H(X)$ such that if $h \in \mathcal{P}, t \in I, h_{t}=\varphi(h, t)$, and $Y \in \mathcal{Y}$, the following conditions hold: (1) $h=\mathrm{id}$ implies $h_{t}=\mathrm{id}$; (2) $h_{0}=h$; (3) $h_{1}=\mathrm{id}$; (4) $h Y=Y$ implies $h_{t} Y=Y$; and (5) if $h Y=Y$ and if $h \mid Y$ is (a) id, (b) LIP, (c) $L-\mathrm{BL}$, (d) LQS, (e) locally $\eta-\mathrm{QS}$, or (f) $\eta-\mathrm{QS}$, then $h_{t} \mid Y$ is, respectively, (a) id, (b) LIP, (c) $L^{*}-\mathrm{BL}$, (d) LQS, (e) (if $\operatorname{dim} Y \geq 2$ ) locally $\eta^{*}-\mathrm{QS}$, or (f) $\eta^{*}-\mathrm{QS}$ with $L^{*}$ depending only on $(L, n)$ and $\eta^{*}$ only on $(\eta, n)$. 
Proof. Let $\mathcal{P}_{0}$ be the set and $\psi: \mathcal{P}_{0} \times I \rightarrow H(X)$ the map given by $\mathcal{D}^{*}(X ; \emptyset, \emptyset$, $\left.X, \bar{B}^{n} \cap X, 2 \bar{B}^{n} \cap X ; \mathcal{Y}\right)$. Let $\mathcal{P}=\mathcal{P}_{0} \cap H(X)$. For $h \in \mathcal{P}$, let $g=\psi(h, 1) \in H(X)$. Then $g=h$ on $\bar{B}^{n} \cap X$ and $d(g$, id $)<\infty$. Set

$$
\varphi(h, 1-t)(x)=t g\left(t^{-2} g^{-1} h(t x)\right) \text { for } 0<t \leq 1, \quad x \in X,
$$

and $\varphi(h, 1)=\mathrm{id}$. Then $\varphi$ is the desired map.

8.3. Remarks. 1. An analogue of (5b) and (5c) for $\Lambda$-isotopies can be added to 8.2. Now $L^{*}$ may depend also on $t$. The case of locally $L$-BL $\Lambda$-isotopies also applies.

2. A more elementary proof for 8.2 (with 8.3 .1 ) in the case $(X, \mathcal{Y})=\left(\mathbf{R}^{n}\right.$, $\left.\left\{\mathbf{R}^{0}, \mathbf{R}^{1}, \ldots, \mathbf{R}^{n}\right\}\right)$ is provided by a construction of the bounded homeomorphism $g$ using the method of [EK, Section 8], and then also the dimensional restriction in (5e) can be dropped. Moreover, choosing in this proof the auxiliary maps from $\mathrm{CAT}=\mathrm{PL}$ or DIFF, we can add to the condition (5) of 8.2 a part $(\mathrm{g})$ where $h_{t} \mid Y$ is a CAT embedding whenever $h \mid Y$ is a CAT embedding. In this way and with $Y=\mathbf{R}^{n},(5 \mathrm{~g})$ was observed by Gauld [Ga, Proposition 1] and (5b) by J. Väisälä (unpublished).

8.4. Theorem. Let $Q$ be a compact LIP manifold, let $P$ be a closed locally LIP flat submanifold of $Q$ with $P \cap \partial Q=\partial P$, and let $M=\operatorname{Int} Q$ and $N=$ Int $P$. Then there exist a neighbourhood $\mathcal{P}$ of id in $H(M)$ and a continuous map $\varphi: \mathcal{P} \times I \rightarrow H(M)$ satisfying the conditions (1)-(6), (7a), and (8a) of 3.2 in the case $X=U=B=B^{\prime}$ if $(M,\{M, N\})$ is substituted for $(X, \mathcal{Y})$.

Proof. We make use of a LIP collar of $(\partial Q, \partial P)$ in $(Q, P)$, provided by an obvious modification of the proof of [LV, 7.4]. So, let $c: \partial Q \times[0,1) \rightarrow Q$ be an open LIP embedding such that $c(x, 0)=x$ for each $x \in \partial Q$ and such that $c^{-1} P=\partial P \times[0,1)$. Then $B=M \backslash c\left[\partial Q \times\left[0, \frac{1}{2}\right)\right]$ is compact. By $\mathcal{D}(M ;\{M, N\})$, valid by 7.3 , it suffices to prove the theorem with $\mathcal{P}=H(M, B)$. We then prove the theorem with $\varphi[\mathcal{P} \times I] \subset \mathcal{P}$. In this way we may replace $M$ by $\partial Q \times(0,1)$, $N$ by $\partial P \times(0,1)$, and $B$ by $\partial Q \times\left[\frac{1}{2}, 1\right)$.

Define a LIP homeomorphism $\delta_{t}: \partial Q \times(0, t) \rightarrow \partial Q \times(0,1)$ for $t \in(0,1]$ by $\delta_{t}(x, s)=(x, s / t)$. If $h \in \mathcal{P}$, we define $\varphi(h, 1-t)=h_{1-t} \in \mathcal{P}$ for $t \in(0,1]$ by

$$
h_{1-t}(x)= \begin{cases}\delta_{t}^{-1} h \delta_{t}(x) & \text { if } x \in \partial Q \times(0, t) \\ x & \text { if } x \in \partial Q \times(t / 2,1)\end{cases}
$$

and set $\varphi(h, 1)=h_{1}=$ id. Since $\delta_{t}^{-1}$ is 1-Lipschitz and $d\left(\delta_{t}^{-1}, \delta_{t^{\prime}}^{-1}\right) \leq\left|t-t^{\prime}\right|$ if $t, t^{\prime} \in(0,1]$, we get that if $h, h^{\prime} \in \mathcal{P}$ and $(x, s, t),\left(x^{\prime}, s^{\prime}, t^{\prime}\right) \in \partial Q \times(0,1) \times I$ with $s<t, s^{\prime}<t^{\prime}$, then

$$
d\left(h_{1-t}(x, s), h_{1-t^{\prime}}^{\prime}\left(x^{\prime}, s^{\prime}\right)\right) \leq d\left(h(x, s / t), h^{\prime}\left(x^{\prime}, s^{\prime} / t^{\prime}\right)\right)+\left|t-t^{\prime}\right| .
$$


It follows that $\varphi$ is continuous. Clearly $\varphi$ satisfies (1)-(6) and (8a). To see (7a), use the above inequality to establish that $\varphi^{*}(f) \mid\{(x, s, \lambda, t) \in \partial Q \times(0,1) \times \Lambda \times I \mid$ $(x, s) \in Y, s<1-t\}$ is locally Lipschitz, and note that $\varphi^{*}(f)^{-1}=\varphi^{*}\left(f^{-1}\right)$.

8.5. Remark. If $\varphi$ in 8.4 is constructed as in the proof, the continuous map $\varphi^{*}: \mathcal{P} \rightarrow I(M)$ is also continuous in the uniform topologies .

8.6. CAT isotopies. We let CAT denote either LIP or LQS. Let $X$ and $\Lambda$ be metric spaces, $U \subset X$ open, and $f=\left(f_{\lambda}\right)_{\lambda \in \Lambda} \in I_{\Lambda}(U ; X)$. We call $f$ a CAT $\Lambda$-isotopy if $f: U \times \Lambda \rightarrow X \times \Lambda$ is LIP whenever CAT $=$ LIP or if $f_{\lambda}: U \rightarrow X$ is LQS for each $\lambda \in \Lambda$ whenever CAT $=$ LQS. A bijective isotopy $h=\left(h_{t}\right)_{t \in I}: X \times I \rightarrow X \times I$ with $h_{0}=$ id is called an ambient isotopy of $X$; then $h$ is said to be supported by a set $D \subset X$ if $h \mid(X \backslash D) \times I=$ id.

We give three consequences of 7.3 to isotopies. The first of them deals with covering CAT isotopies by ambient CAT isotopies.

8.7. Theorem. Let CAT, $M, \mathcal{Y}$ be as in Case 1 or in Case 2 of 7.2, let $U \subset M$ be open, $B \subset U$ compact, $f: U \times I \rightarrow M \times I$ a CAT isotopy, and $D$ a neighbourhood of the compact set $\bigcup_{t \in I} f_{t} B$ in $M$. Then there is an ambient CAT isotopy $h$ of $M$ supported by $D$ such that $f=h\left(f_{0} \times \mathrm{id}\right)$ on $B \times I$ and such that the following condition holds:

(1) Let $Y \in \mathcal{Y}$. If $f$ respects $Y \times I$, so does $h$. If, in addition, $f=f_{0} \times$ id on $(U \cap Y) \times I$, then $h \mid Y \times I=\mathrm{id}$.

This CAT analogue of [EK, 1.2] is proved as [EK, 1.2] is proved.

For CAT = LIP, 8.7 (without (1)) is already given in [SS, p. 519]. A generalization of 8.7 to the case of a noncompact $B$ and of a general parameter space $\Lambda$ can be obtained straightforwardly modifying [Si, 6.5 and 6.6].

8.8. Theorem. Let CAT, $M, \mathcal{Y}$ be as in 8.7, let $B \subset M$ be compact, and let $\mathcal{U}$ be an open cover of $B$ in $M$. Then every ambient CAT isotopy $f$ of $M$ supported by $B$ can be written as $f=f^{(1)} f^{(2)} \ldots f^{(k)}$ where each $f^{(j)}$ is an ambient CAT isotopy of $M$ supported by some member of $\mathcal{U}$ such that for each $Y \in \mathcal{Y}$, if $f$ respects $Y \times I$ or is the identity on $Y \times I$, so does each $f^{(j)}$, respectively.

This CAT analogue of $[\mathrm{EK}, 1.3]$ (where $B=M$ ) is proved as [EK, 1.3] is proved.

8.9. Locally CAT flat isotopies. For the last application to isotopies we need two more notions. Let $M$ be a CAT $n$-manifold, $N$ a CAT $p$-manifold $(0 \leq p \leq$ $n$ ), and $f: N \times I \rightarrow M \times I$ an embedding of the form $(x, t) \mapsto\left(f_{t}(x), t\right)$ such that $f$ is LIP if CAT $=$ LIP, such that $f_{t}: N \rightarrow M$ is LQS for each $t \in I$ if CAT $=\mathrm{LQS}$, and such that $f^{-1}[\partial M \times I]=N_{0} \times I$ where $N_{0}$ is a subset of $N$ if $p=0$ or a locally CAT flat $(p-1)$-submanifold of $\partial N$ if $p \geq 1$. (Note that unless 
$p=n$ and $N_{0}=\partial N, f$ is not open and, thus, is not a (CAT) isotopy in our terminology.) We call $f$ a locally CAT flat isotopy if for each point $(x, t) \in N \times I$ there exist a neighbourhood $J$ of $t$ in $I$, an open subset $U$ of $\mathbf{R}^{n}$ (whenever $x \notin N_{0}$ and either $p=0$ or $x \in \partial N$ with $p=1$ ) or of $\mathbf{R}_{+}^{n}$ (in the other cases), and CAT $J$-isotopies $\alpha:\left(U \cap Y_{n, p}\right) \times J \rightarrow N \times J$ and $\beta: U \times J \rightarrow M \times J$ onto open neighbourhoods of $(x, t)$ in $N \times J$ and of $f(x, t)$ in $M \times J$, respectively, such that $\beta$ extends $f \alpha$. In this case, $f_{t} N$ is a locally CAT flat submanifold of $M$ for each $t \in I$.

Suppose that in the introduction of $M, N$, and $f$ above, $N$ is a locally CAT flat submanifold of $M$ and $N_{0}=N \cap \partial M$. Then we call $f$ a locally CAT extendible isotopy if for each point $(x, t) \in N \times I$ there exist a neighbourhood $J$ of $t$ in $I$, an open neighbourhood $V$ of $x$ in $M$, and a CAT $J$-isotopy $g: V \times J \rightarrow$ $M \times J$ extending $f \mid(V \cap N) \times J$. It is easy to see that if $f$ is a locally CAT extendible isotopy, then $f$ is a locally CAT flat isotopy, and that in the LIP case the converse also holds (in the LQS case the converse is open).

We can now state a CAT analogue of the theorem [EK, 1.4] on extension of locally flat isotopies of topological manifolds.

8.10. Theorem. Suppose that $f: N \times I \rightarrow M \times I$ is either a locally LIP flat isotopy of a compact LIP manifold $N$ into a LIP manifold $M$ or a locally LQS extendible isotopy of a compact locally LQS flat submanifold $N$ of an LQS manifold $M$ into $M$. Let CAT be, respectively, LIP or LQS, and let $D$ be a neighbourhood of $\bigcup_{t \in I} f_{t} N$ in $M$. Then there is an ambient CAT isotopy $h$ of $M$ supported by $D$ such that $f=h\left(f_{0} \times \mathrm{id}\right)$.

The proof of 8.10 , based on 7.3 and 8.7 , is similar to that of [EK, 1.4].

The following result is our last application. For CAT $=$ TOP it is due to [CK].

8.11. Theorem. Let CAT be LIP or LQS. Then there are only countably many CAT homeomorphism classes of compact CAT manifolds.

Proof. By the CAT embedding result [LT, 4.6], it suffices to show that for all integers $n \geq 0$ and $k \geq k_{0} \geq 0$ there are only countably many CAT homeomorphism classes of compact connected CAT $n$-submanifolds $M$ of $\mathbf{R}^{2 n+1}$ for which there are CAT embeddings $h_{i}: 2 \bar{B}_{+}^{n} \rightarrow M\left(1 \leq i \leq k_{0}\right)$ with $h_{i}^{-1} \partial M=2 \bar{B}^{n-1}$ and $h_{i}: 2 \bar{B}^{n} \rightarrow \operatorname{Int} M\left(k_{0}<i \leq k\right)$ such that the sets $h_{i} \bar{B}_{+}^{n}\left(i \leq k_{0}\right)$ and $h_{i} \bar{B}^{n}$ $\left(i>k_{0}\right)$ cover $M$. We can then follow the proof in [CK] for manifolds with empty boundary; we only resort to $\mathcal{D}^{*}\left(\mathbf{R}_{+}^{n} ;\left\{\mathbf{R}_{+}^{n}\right\}\right)$ and $\mathcal{D}^{*}\left(\mathbf{R}^{n} ;\left\{\mathbf{R}^{n}\right\}\right)$. 口

8.12. Remark. For CAT manifolds $M$ with $\operatorname{dim} M \neq 4 \neq \operatorname{dim} \partial M, 8.11$ also follows from the TOP version by the CAT Hauptvermutung ([ $\mathrm{Su}_{1}$, Corollary 3], $\left[\mathrm{TV}_{2}, 4.5\right.$ and 4.8]). On the other hand, Donaldson and Sullivan ([DS, Theorem 2], cf. [Su $\mathrm{Su}_{2}$, p. 1221]) have recently shown that there are compact LIP 
(or even complex algebraic) 4-manifolds without boundary which are homeomorphic but not LQS homeomorphic.

\section{References}

[AVV] Anderson, G. D., M. K. Vamanamurthy, and M. Vuorinen: Dimension-free quasiconformal distortion in $n$-space. - Trans. Amer. Math. Soc. 297, 1986, 687-706.

[CK] Cheeger, J., and J. M. Kister: Counting topological manifolds. - Topology 9, 1970, $149-151$.

[Ch] Chernavskir , A. V.: Local contractibility of the group of homeomorphisms of a manifold. - Mat. Sb. (N.S.) 79 (121), 1969, 307-356 = Math. USSR-Sb. 8, 1969, 287-333.

[DS] Donaldson, S. K., and D. P. Sullivan: Quasiconformal 4-manifolds. - To appear.

[Du] Dugundji, J.: Topology. - Allyn and Bacon, Inc., Boston, 1966.

[Ed] EDWARDS, R. D.: Wrapping small homeomorphisms around tori. - Topology of manifolds: Proceedings of the University of Georgia Topology of Manifolds Institute 1969, edited by J. C. Cantrell and C. H. Edwards, Jr., Markham Publishing Company, Chicago, 1970, 4.

[EK] Edwards, R. D., and R. C. Kirby: Deformations of spaces of imbeddings. - Ann. of Math. (2) 93, 1971, 63-88.

[Ga] GAULD, D. B.: Local contractibility of $\mathrm{PL}(M)$ for a compact manifold. - Math. Chronicle 4, 1975, 1-6.

[GV] Gauld, D. B., and M. K. Vamanamurthy: Quasiconformal extensions of mappings in $n$-space. - Ann. Acad. Sci. Fenn. Ser. A I Math. 3, 1977, 229-246.

[Ki] Kirby, R. C.: Stable homeomorphisms and the annulus conjecture. - Ann. of Math. (2) 89, 1969, 575-582.

[KS] Kirby, R. C., and L. C. Siebenmann: Foundational essays on topological manifolds, smoothings, and triangulations. - Anr. of Math. Stud. 88, Princeton University Press, Princeton, N. J., 1977.

[Ku] KuUsalo, T.: Generalized conformal capacity and quasiconformal metrics. - Proceedings of the Romanian-Finnish Seminar on Teichmüller Spaces and Quasiconformal Mappings, Braşov, 1969, edited by C. Andreian Cazacu, Publishing House of the Academy of the Socialist Republic of Romania, Bucharest, 1971, 193-202.

[Lu] Luukkainen, J.: Deformation of one-dimensional quasisymmetric embeddings. - Proceedings of the XIII Rolf Nevanlinna Colloquium, Joensuu, 1987, edited by I. Laine, S. Rickman and T. Sorvali, Lecture Notes in Math. 1351, Springer-Verlag, 236-242 (to appear).

[LT] LuUkKainen, J., and P. Tukia: Quasisymmetric and Lipschitz approximation of embeddings. - Ann. Acad. Sci. Fenn. Ser. A I Math. 6, 1981, 343-367.

[LV] LuUkKaInen, J., and J. VÄISÄLÄ: Elements of Lipschitz topology. - Ibid. 3, 1977, 85-122.

[RS] Rourke, C. P., and B. J. SANDerson: Introduction to piecewise-linear topology. - Springer-Verlag, Berlin-Heidelberg-New York, 1972.

[Si] Siebenmann, L. C.: Deformation of homeomorphisms on stratified sets. - Comment. Math. Helv. 47, 1972, 123-163.

[SS] Siebenmann, L., and D. Sullivan: On complexes that are Lipschitz manifolds. - Geometric topology: Proceedings of the Georgia Topology Conference, Athens, Ga., 1977, edited by J. C. Cantrell, Academic Press, New York-San Francisco-London, 1979, $503-525$.

[Su $\mathrm{Su}_{1}$ Sullivan, D.: Hyperbolic geometry and homeomorphisms. - Ibid., 543-555.

[Su $\left.\mathrm{Su}_{2}\right]$ Sullivan, D.: Quasiconformal homeomorphisms in dynamics, topology, and geometry. Proceedings of the International Congress of Mathematicians, Berkeley, California, 
USA, 1986, edited by A. M. Gleason, American Mathematical Society, 1987, 12161228.

[TV 1 TUKIA, P., and J. VÄISÄLÄ: Quasisymmetric embeddings of metric spaces. - Ann. Acad. Sci. Fenn. Ser. A I Math. 5, 1980, 97-114.

[TV $\left.{ }_{2}\right]$ TUKIA, P., and J. VÄISÄLÄ: Lipschitz and quasiconformal approximation and extension. - Ibid. 6, 1981, 303-342.

$\left[\mathrm{TV}_{3}\right]$ TUKIA, P., and J. VÄIsÄLÄ: Quasiconformal extension from dimension $n$ to $n+1$. Ann. of Math. (2) 115, 1982, 331-348.

[Vä1] VÄISÄL $\ddot{A}$, J.: Lectures on $n$-dimensional quasiconformal mappings. - Lecture Notes in Math. 229, Springer-Verlag, Berlin-Heidelberg-New York, 1971.

[Vä] VÄIs ̈̈L̈̈, J.: Quasi-symmetric embeddings in Euclidean spaces. - Trans. Amer. Math. Soc. 264, 1981, 191-204.

University of Helsinki

Department of Mathematics

Hallituskatu 15

SF-00100 Helsinki

Finland

Received 25 September 1987 